\title{
Black holes in presence of cosmological constant: second order in $\frac{1}{D}$
}

\author{
Sayantani Bhattacharyya, ${ }^{a, b}$ Parthajit Biswas ${ }^{a, b}$ and Yogesh Dandekar ${ }^{c}$ \\ ${ }^{a}$ National Institute of Science Education and Research (NISER), \\ Bhubaneswar, P.O. Jatni, Khurda 752050, Odisha, India \\ ${ }^{b}$ Homi Bhabha National Institute, Training School Complex, \\ Anushakti Nagar, Mumbai 400085, India \\ ${ }^{c}$ Department of Theoretical Physics, Tata Institute of Fundamental Research, \\ Homi Bhabha Road, Mumbai 400005, India \\ E-mail: sayanta@niser.ac.in, parthajit.biswas@niser.ac.in, \\ yogesh@theory.tifr.res.in
}

ABSTRACT: We have extended the results of [1] upto second subleading order in an expansion around large dimension $D$. Unlike the previous case, there are non-trivial metric corrections at this order. Due to our 'background-covariant' formalism, the dependence on Ricci and the Riemann curvature tensor of the background is manifest here. The gravity system is dual to a dynamical membrane coupled with a velocity field. The dual membrane is embedded in some smooth background geometry that also satisfies the Einstein equation in presence of cosmological constant. We explicitly computed the corrections to the equation governing the membrane-dynamics. Our results match with earlier derivations in appropriate limits. We calculated the spectrum of QNM from our membrane equations and matched them against similar results derived from gravity.

Keywords: Black Holes, Classical Theories of Gravity

ARXIV EPRINT: 1805.00284 


\section{Contents}

1 Introduction 2

2 Set up and final result $\quad 3$

2.1 Solution at the first subleading order 4

2.2 Final result: metric and membrane equation at second subleading order 5

$\begin{array}{lll}3 & \text { Sketch of the computation } & 7\end{array}$

4 Checks 11

$\begin{array}{ll}\text { 4.1 Check for internal consistency } & 12\end{array}$

$\begin{array}{lll}\text { 4.1.1 Vanishing of } \mathcal{E}^{(2)} & 13\end{array}$

$\begin{array}{ll}\text { 4.1.2 Vanishing of } \mathcal{E}^{(t r)} & 13\end{array}$

$\begin{array}{ll}\text { 4.1.3 Vanishing of } \mathcal{E}_{B}^{\left(V_{2}\right)} & 13\end{array}$

4.2 Quasinormal modes for Schwarzschild black hole in background AdS/dS spacetime 13

$\begin{array}{ll}\text { 4.3 Quasinormal modes for AdS Schwarzschild black brane } & 18\end{array}$

$\begin{array}{lll}5 & \text { Future directions } & 21\end{array}$

A Calculation of the sources $-S_{A B} \quad 22$

B Some identities $\quad 31$

B.1 The derivation of the identity (4.3) 31

B.2 The derivation of scalar structure $\mathfrak{s}_{2}(3.16) \quad 32$

B.3 The derivation of the identity (A.9) 33

B.4 The derivation of the identity (A.38) 34

B.5 The derivation of the identity (A.39) 36

C QNM for AdS/dS Schwarzschild Black hole: details of the calculation 39

C.1 Computation of $\mathcal{K}_{\mu \nu}$

C.2 Computation of the terms relevant for the membrane equation 42

C.3 Arguments leading to (4.13) 43

D QNM for AdS Schwarzschild black brane: details of the calculation 43

D.1 Computation of $\mathcal{K}_{\mu \nu}$

D.2 Computation of the terms relevant for membrane equation 45

D.3 Arguments leading to (4.32) 46 


\section{Introduction}

Recently it has been shown that in large number of dimensions, black hole solutions simplify a lot. ${ }^{1}$ The effect of the black hole is essentially confined around its event horizon in a parametrically thin region whose thickness is proportional to the inverse of the number of dimension. Further, the spectrum of the linearized fluctuation (Quasi Normal Modes or QNMs) develops a large gap proportional to the number of dimension. In [2] authors have shown how one can formulate the autonomous nonlinear theory of the low lying modes. They combine to form a dynamical black hole solution to Einstein equation which could be determined in an expansion in inverse powers of $D$.

In [1] the authors have extended the calculation of [2] (which was for pure Einstein Gravity) to solutions in presence of cosmological constant and in general for any asymptotic background provided it is a solution of the gravity equation. The method used in [1] has manifest background covariance but the calculation were done only upto the first subleading order in $\left(\frac{1}{D}\right)$.

In this note, we would like to extend the calculation of [1] to the second subleading order. The key motivation is two-fold. Firstly from the result of [1] we know that at the first subleading order the background curvature does not appear explicitly in any of the equation or the solution. However it should appear explicitly at second subleading order (which, very roughly speaking, captures the effect of two derivatives on the background). Secondly from the experience of the 'flat space computation', it is expected that at this order we should see the entropy production from a dynamical black hole.

However, in this note we shall confine ourselves only to the computation of the membrane equation of motion and the metric correction upto the second subleading order in $\left(\frac{1}{D}\right)$ expansion. We leave the 'study of entropy production' for future.

As a consistency check of our results we shall linearize our membrane equation and compare the spectrum with that of the low lying QNMs (already determined in [16]). We shall find a perfect match upto the relevant order.

The organization of this note is as follows. In section 2 we have described the basic set-up of our problem in terms of equations and also the final result for the corrections to metric and the membrane equations. Next in section 3 we gave a sketch of the computation, which turns out to be quite tedious in this case. Many of the details we collected in the appendices. In section 4 we have performed several checks. Some of them are about the internal consistency of our set of equations (see subsection 4.1) and the rest are about the calculation of the linearized spectrum of our membrane around different static backgrounds. We have also matched them against the known results of QNMs (see subsection 4.2). Finally in section 5 we discuss about the future directions.

\footnotetext{
${ }^{1}$ See [1-7] for the work related to the formulation of Membrane paradigm at large D. See [8-11] and also [12-14] for initial work introducing the large D limit in General Relativity. See [15-37] for other parallel work which uses the technique of large D expansion.
} 


\section{Set up and final result}

In this section we shall briefly define the basic set-up of our problem in terms of equations. It is essentially an extension of section- 2 of [1]. So we shall be very brief here.

We are dealing with pure gravity in presence of cosmological constant. The Action and the equation of motion are given by the following.

$$
\mathcal{S}=\int \sqrt{-G}[\mathcal{R}-\Lambda]
$$

Where the dimension (denoted as $D$ ) dependence of $\Lambda$ is parametrized as follows

$$
\Lambda=[(D-1)(D-2)] \lambda, \quad \lambda \sim \mathcal{O}(1)
$$

Varying (2.1) with respect to the metric we get the equation of motion

$$
E_{A B} \equiv R_{A B}-\left(\frac{\mathcal{R}-\Lambda}{2}\right) G_{A B}=0
$$

Our aim is to solve these equations perturbatively as a series in inverse power of $D$. Schematically our solution will take the form

$$
G_{A B}=G_{A B}^{(0)}+\left(\frac{1}{D}\right) G_{A B}^{(1)}+\left(\frac{1}{D}\right)^{2} G_{A B}^{(2)}+\cdots
$$

We take our starting ansatz $G_{A B}^{(0)}$ to be the following ${ }^{2}$

$$
G_{A B}^{(0)}=g_{A B}+\psi^{-D} O_{A} O_{B}
$$

Here $g_{A B}$ is the background metric which could be any smooth solution of the starting equation (2.1).

$O \equiv O_{A} d X^{A}$ is a one-form that is null with respect to the background metric $g_{A B}$.

It turns out that this starting solution has an event horizon, given by the null hypersurface $-\mathcal{S}: \psi=1$. We define the function $\psi$ in a way so that $\psi=1$ is the horizon to all order in $\left(\frac{1}{D}\right)$ expansion. Further it satisfies the following equation (which we shall refer to as 'subsidiary condition-1' $)^{3}$

$$
\nabla^{2} \psi^{-D}=0
$$

We can always determine $\psi$ explicitly in an expansion in $\left(\frac{1}{D}\right)$ solving equation (2.6) with the initial condition that $\psi=1$ coincides with the horizon [6]. We fix the normalization of $O^{A}$ by demanding that the inner product between $O_{A}$ and the unit normal to the $\psi=$ constant surface (viewed as a hypersurface embedded in the background $g_{A B}$ ) is always one. In terms of equation this implies

$$
O \cdot n \equiv\left[\frac{O \cdot \partial \psi}{\sqrt{(\partial \psi) \cdot(\partial \psi)}}\right]=1
$$

\footnotetext{
${ }^{2}$ See [1-3] for detailed explanation for this choice.

${ }^{3}$ Throughout this note '.' denotes the contraction with respect to the background $g_{A B}$ and $\nabla$ is a covariant derivative with respect to the background. All raising and lowering of indices will also be with respect to the background. Otherwise it would be written explicitly.
} 
Note that using the above normalization we can define a unit normalized velocity field $u^{A}$.

$$
u^{A} \equiv-\left(O^{A}-n^{A}\right), \quad u \cdot u=-1
$$

It turns out that $u^{A}$ is the null generator of the $\psi=1$ hypersurface (viewed as a null hypersurface embedded in $\left.G_{A B}^{(0)}\right)$.

However, just the normalization cannot fix all components of the null one-form $O_{A}$ everywhere. We fix this ambiguity demanding that $O_{A}$ satisfies the following geodesic constraint ( which we shall refer to as 'subsidiary condition-2')

$$
(O \cdot \nabla) O_{A} \propto O_{A}, \quad O \cdot O=0
$$

(2.9) again could be solved in an expansion in $\left(\frac{1}{D}\right)$ provided we have an unambiguous initial condition to all order. We fix this condition by demanding that $u^{A}$ as defined in (2.8) is the null generator of the horizon to all order [6].

We shall determine the metric corrections in terms of the well defined $\psi$ and $O_{A}$ fields and their derivatives.

\subsection{Solution at the first subleading order}

As mentioned in the introduction, $G_{A B}^{(1)}$ - the metric correction at first subleading order has already been determined [1]. For convenience, here we shall quote the first order solution.

It turns out that Einstein equations could be solved provided the extrinsic curvature of the $\psi=1$ hypersurface (viewed as a hypersurface embedded in the background) and the velocity field $u^{A}$ together satisfy the following constraint equations on the horizon. The constraint equation can be written as an intrinsic equation to the membrane.

$$
\begin{aligned}
\mathcal{P}_{\mu}^{\nu}\left[\frac{\hat{\nabla}^{2} u_{\nu}}{\mathcal{K}}-\frac{\hat{\nabla}_{\nu} \mathcal{K}}{\mathcal{K}}+u_{\alpha} \mathcal{K}_{\nu}^{\alpha}-(u \cdot \hat{\nabla}) u_{\nu}\right] & =\mathcal{O}\left(\frac{1}{D}\right), \quad \hat{\nabla} \cdot u=\mathcal{O}\left(\frac{1}{D}\right) \\
\text { where } \quad \mathcal{P}_{\mu \nu} & =\hat{g}_{\mu \nu}+u_{\mu} u_{\nu}
\end{aligned}
$$

Here $\hat{g}_{\mu \nu}$ denotes the induced metric on the membrane $(\psi=1$ hypersurface) and $\hat{\nabla}$ is the covariant derivative with respect to $\hat{g}_{\mu \nu}$. The velocity field $u_{\mu}$ is the pull back of the bulk velocity field $u_{A}$ and $\mathcal{K}_{\mu \nu}$ is the pull back of the extrinsic curvature of the membrane onto the hypersurface. ${ }^{4} \mathcal{K}$ is the trace of the extrinsic curvature.

\footnotetext{
${ }^{4}$ In terms of equations what we mean is the following.

The space time form of the extrinsic curvature is given by

$$
K_{A B}=\Pi_{A}^{C} \nabla_{A} n_{B}, \text { where } \Pi_{A B}=g_{A B}-n_{A} n_{B}
$$

$u_{\mu}$ and $\mathcal{K}_{\mu \nu}$ is defined as

$$
u_{\mu}=\left(\frac{\partial X^{A}}{\partial y^{\mu}}\right) u_{A}, \quad \mathcal{K}_{\mu \nu}=\left(\frac{\partial X^{M}}{\partial y^{\mu}}\right)\left(\frac{\partial X^{N}}{\partial y^{\nu}}\right) K_{M N}
$$

where $X^{M}$ denotes the coordinates of the full space time and $y^{\mu}$ denotes coordinates on the membrane.
} 
For every solution of the above constraint equations we could determine $G_{A B}^{(1)}$. It turns out $G_{A B}^{(1)}$ simply vanishes given our choice of subsidiary conditions.

In this note our goal is to find corrections to equation (2.10) to the next order in $\left(\frac{1}{D}\right)$ expansion and also $G_{A B}^{(2)}$.

But before getting into any details of the computation, we shall first present our final result.

\subsection{Final result: metric and membrane equation at second subleading order}

In this subsection we shall present the subleading correction to the membrane equation (2.10) and the solution to $G_{A B}^{(2)}$.

The metric correction would take the following form.

$$
G_{A B}^{(2)}=\left[O_{A} O_{B}\left(\sum_{n=1}^{2} f_{n}(R) \mathfrak{s}_{n}\right)+t(R) \mathfrak{t}_{A B}+v(R)\left(\mathfrak{v}_{A} O_{B}+\mathfrak{v}_{B} O_{A}\right)\right]
$$

where $R \equiv D(\psi-1), \quad P_{A B}=g_{A B}-n_{A} n_{B}+u_{A} u_{B}$

and, $n^{A} \mathfrak{v}_{A}=u^{A} \mathfrak{v}_{A}=0, \quad n^{A} \mathfrak{t}_{A B}=u^{A} \mathfrak{t}_{A B}=0, \quad g^{A B} \mathfrak{t}_{A B}=0$

where

$$
\begin{aligned}
& \mathfrak{t}_{A B}=P_{A}^{C} P_{B}^{D}\left[\bar{R}_{F C D E} O^{E} O^{F}+\frac{K}{D}\left(K_{C D}-\frac{\nabla_{C} u_{D}+\nabla_{D} u_{C}}{2}\right)\right. \\
& \left.-P^{E F}\left(K_{E C}-\nabla_{E} u_{C}\right)\left(K_{F D}-\nabla_{F} u_{D}\right)\right] \\
& \mathfrak{v}_{A}=P_{A}^{B}\left[\frac{K}{D}\left(n^{D} u^{E} O^{F} \bar{R}_{F B D E}\right)+\frac{K^{2}}{2 D^{2}}\left(\frac{\nabla_{B} K}{K}+(u \cdot \nabla) u_{B}-2 u^{D} K_{D B}\right)\right. \\
& \left.-P^{F D}\left(\frac{\nabla_{F} K}{D}-\frac{K}{D}\left(u^{E} K_{E F}\right)\right)\left(K_{D B}-\nabla_{D} u_{B}\right)\right] \\
& \mathfrak{s}_{1}=u^{E} u^{F} n^{D} n^{C} \bar{R}_{C E F D}+\left(\frac{u \cdot \nabla K}{K}\right)^{2}+\frac{\tilde{\nabla}_{A} K}{K}\left[4 u^{B} K_{B}^{A}-2\left[(u \cdot \nabla) u^{A}\right]-\frac{\tilde{\nabla}^{A} K}{K}\right] \\
& -\left(\tilde{\nabla}_{A} u_{B}\right)\left(\tilde{\nabla}^{A} u^{B}\right)-(u \cdot K \cdot u)^{2}-\left[(u \cdot \tilde{\nabla}) u_{A}\right]\left[(u \cdot \tilde{\nabla}) u^{A}\right]+2\left[(u \cdot \nabla) u^{A}\right]\left(u^{B} K_{B A}\right) \\
& -3(u \cdot K \cdot K \cdot u)-\frac{K}{D}\left(\frac{u \cdot \nabla K}{K}-u \cdot K \cdot u\right) \\
& \mathfrak{s}_{2}=\frac{K^{2}}{D^{2}}\left[-\frac{K}{D}\left(\frac{u \cdot \nabla K}{K}-u \cdot K \cdot u\right)-2 \lambda-(u \cdot K \cdot K \cdot u)+2\left(\frac{\nabla_{A} K}{K}\right) u^{B} K_{B}^{A}-\left(\frac{u \cdot \nabla K}{K}\right)^{2}\right. \\
& \left.+2\left(\frac{u \cdot \nabla K}{K}\right)(u \cdot K \cdot u)-\left(\frac{\tilde{\nabla}^{D} K}{K}\right)\left(\frac{\tilde{\nabla}_{D} K}{K}\right)-(u \cdot K \cdot u)^{2}+n^{B} n^{D} u^{E} u^{F} \bar{R}_{F B D E}\right]
\end{aligned}
$$

Where, $\bar{R}_{A B C D}$ is the Riemann tensor ${ }^{5}$ of the background metric $g_{A B}$ and $\tilde{\nabla}$ is defined as

\footnotetext{
${ }^{5}$ Riemann tensor is defined by the relation
}

$$
\left[\nabla_{A}, \nabla_{B}\right] \omega_{C}=R_{A B C}{ }^{D} \omega_{D} .
$$


follows: for any general tensor with $n$ indices $W_{A_{1} A_{2} \cdots A_{n}}$

$$
\begin{aligned}
\tilde{\nabla}_{A} W_{A_{1} A_{2} \cdots A_{n}}= & \Pi_{A}^{C} \Pi_{A_{1}}^{C_{1}} \Pi_{A_{2}}^{C_{2}} \cdots \Pi_{A_{n}}^{C_{n}}\left(\nabla_{C} W_{C_{1} C_{2} \cdots C_{n}}\right), \text { with } \Pi_{A B}=g_{A B}-n_{A} n_{B} \\
t(R)= & -2\left(\frac{D}{K}\right)^{2} \int_{R}^{\infty} \frac{y d y}{e^{y}-1} \\
v(R)= & 2\left(\frac{D}{K}\right)^{3}\left[\int_{R}^{\infty} e^{-x} d x \int_{0}^{x} \frac{y e^{y}}{e^{y}-1} d y-e^{-R} \int_{0}^{\infty} e^{-x} d x \int_{0}^{x} \frac{y e^{y}}{e^{y}-1} d y\right] \\
f_{1}(R)= & -2\left(\frac{D}{K}\right)^{2} \int_{R}^{\infty} x e^{-x} d x+2 e^{-R}\left(\frac{D}{K}\right)^{2} \int_{0}^{\infty} x e^{-x} d x \\
f_{2}(R)= & \left(\frac{D}{K}\right)\left[\int_{R}^{\infty} e^{-x} d x \int_{0}^{x} \frac{v(y)}{1-e^{-y}} d y-e^{-R} \int_{0}^{\infty} e^{-x} d x \int_{0}^{x} \frac{v(y)}{1-e^{-y}} d y\right] \\
& -\left(\frac{D}{K}\right)^{4}\left[\int_{R}^{\infty} e^{-x} d x \int_{0}^{x} \frac{y^{2} e^{-y}}{1-e^{-y}} d y-e^{-R} \int_{0}^{\infty} e^{-x} d x \int_{0}^{x} \frac{y^{2} e^{-y}}{1-e^{-y}} d y\right]
\end{aligned}
$$

As we can see that our solution is parametrized by the shape of the constant $\psi$ hypersurfaces (encoded in its extrinsic curvature $K_{A B}$ ) along with the velocity field $u^{A}$. However, because of our subsidiary conditions if we know $K_{A B}$ and $u^{A}$ along one constant $\psi$ hypersurface, they are determined everywhere else. In this sense the real data our class of solutions are to be provided only along one simple surface; the most natural choice of which is the horizon or the hypersurface $\psi=1$.

As we have mentioned before, we cannot choose any arbitrary shape of the membrane and velocity field as our initial data. The metric, presented above, would solve Einstein equation (2.3) only if the data satisfy some constraint - the equation (2.10) with subleading corrections. This will lead to the following corrected membrane equation at this order.

$$
\begin{aligned}
& {\left[\frac{\hat{\nabla}^{2} u_{\alpha}}{\mathcal{K}}-\frac{\hat{\nabla}_{\alpha} \mathcal{K}}{\mathcal{K}}+u^{\beta} \mathcal{K}_{\beta \alpha}-u \cdot \hat{\nabla} u_{\alpha}\right] \mathcal{P}_{\gamma}^{\alpha}+\left[-\frac{u^{\beta} \mathcal{K}_{\beta \delta} \mathcal{K}_{\alpha}^{\delta}}{\mathcal{K}}+\frac{\hat{\nabla}^{2} \hat{\nabla}^{2} u_{\alpha}}{\mathcal{K}^{3}}-\frac{\left(\hat{\nabla}_{\alpha} \mathcal{K}\right)(u \cdot \hat{\nabla} \mathcal{K})}{\mathcal{K}^{3}}\right.} \\
& -\frac{\left(\hat{\nabla}_{\beta} \mathcal{K}\right)\left(\hat{\nabla}^{\beta} u_{\alpha}\right)}{\mathcal{K}^{2}}-\frac{2 \mathcal{K}^{\delta \sigma} \hat{\nabla}_{\delta} \hat{\nabla}_{\sigma} u_{\alpha}}{K^{2}}-\frac{\hat{\nabla}_{\alpha} \hat{\nabla}^{2} \mathcal{K}}{\mathcal{K}^{3}}+\frac{\hat{\nabla}_{\alpha}\left(\mathcal{K}_{\beta \delta} \mathcal{K}^{\beta \delta} \mathcal{K}\right)}{K^{3}}+3 \frac{(u \cdot \mathcal{K} \cdot u)\left(u \cdot \hat{\nabla} u_{\alpha}\right)}{\mathcal{K}} \\
& -3 \frac{(u \cdot \mathcal{K} \cdot u)\left(u^{\beta} \mathcal{K}_{\beta \alpha}\right)}{\mathcal{K}}-6 \frac{(u \cdot \hat{\nabla} \mathcal{K})\left(u \cdot \hat{\nabla}_{\alpha}\right)}{\mathcal{K}^{2}}+6 \frac{(u \cdot \hat{\nabla} \mathcal{K})\left(u^{\beta} \mathcal{K}_{\beta \alpha}\right)}{\mathcal{K}^{2}}+3 \frac{u \cdot \hat{\nabla} u_{\alpha}}{D-3} \\
& \left.-3 \frac{u^{\beta} \mathcal{K}_{\beta \alpha}}{D-3}-\frac{(D-1) \lambda}{\mathcal{K}^{2}}\left(\frac{\hat{\nabla}_{\alpha} \mathcal{K}}{\mathcal{K}}-2 u^{\sigma} \mathcal{K}_{\sigma \alpha}+2(u \cdot \hat{\nabla}) u_{\alpha}\right)\right] \mathcal{P}_{\gamma}^{\alpha}=\mathcal{O}\left(\frac{1}{D}\right)^{2} \\
& \hat{\nabla} \cdot u-\frac{1}{2 \mathcal{K}}\left(\hat{\nabla}_{(\alpha} u_{\beta)} \hat{\nabla}_{(\gamma} u_{\delta)} \mathcal{P}^{\beta \gamma} \mathcal{P}^{\alpha \delta}\right)=\mathcal{O}\left(\frac{1}{D}\right)^{2}
\end{aligned}
$$

Where $\hat{\nabla}$ is the covariant derivative with respect to $\hat{g}_{\mu \nu}$, the induced metric on $\psi=1$ hypersurface. $\mathcal{K}_{\mu \nu}$ and $u_{\mu}$ are defined in $(2.11) . \hat{\nabla}_{(\alpha} u_{\beta)}$ is defined as

$$
\hat{\nabla}_{(\alpha} u_{\beta)} \equiv \hat{\nabla}_{\alpha} u_{\beta}+\hat{\nabla}_{\beta} u_{\alpha}
$$




\section{Sketch of the computation}

It turns out that though the computation to determine the second order metric correction is tedious, conceptually it is a straightforward extension of what has been done in [1]. Therefore in this section, we shall omit most of the derivations and mention only those where there are some differences from [1].

We shall follow the same convention as in [1]. In particular our choice of gauge is also the same, namely

$$
O^{B} G_{A B}^{(2)}=0
$$

With this gauge choice the second order correction could be parametrized as

$$
\begin{aligned}
G_{A B}^{(2)}= & \left(O_{A} O_{B} \sum_{n} f_{n}(R) \mathfrak{s}_{n}+\frac{1}{D} P_{A B} \sum_{n} h_{n}(R) \mathfrak{s}_{n}+\sum_{n} t_{n}(R)\left[\mathfrak{t}_{n}\right]_{A B}\right. \\
& \left.+\sum_{n} v_{n}(R)\left(\left[\mathfrak{v}_{n}\right]_{A} O_{B}+\left[\mathfrak{v}_{n}\right]_{B} O_{A}\right)\right)
\end{aligned}
$$

where $R \equiv D(\psi-1), \quad P_{A B}=g_{A B}-n_{A} n_{B}+u_{A} u_{B}$

and, $n^{A}\left[\mathfrak{v}_{n}\right]_{A}=u^{A}\left[\mathfrak{v}_{n}\right]_{A}=0, \quad n^{A}\left[\mathfrak{t}_{n}\right]_{A B}=u^{A}\left[\mathfrak{t}_{n}\right]_{A B}=0, \quad g^{A B}\left[\mathfrak{t}_{n}\right]_{A B}=0$

Here $\mathfrak{s}_{n},\left[\mathfrak{v}_{n}\right]_{A},\left[\mathfrak{t}_{n}\right]_{A B}$ are different independent scalar, vector and tensor structures, constructed out of the membrane data.

Evaluating (2.3) on $\left[G_{A B}=G_{A B}^{(0)}+\left(\frac{1}{D}\right) G_{A B}^{(1)}+\left(\frac{1}{D}\right)^{2} G_{A B}^{(2)}+\mathcal{O}\left(\frac{1}{D}\right)^{3}\right]$ upto order $\mathcal{O}(1)$, we got a set of coupled, ordinary but inhomogeneous differential equation for the unknown functions in equation (3.1). Boundary conditions for these differential equations are set by the following physical conditions.

1. The surface $(\psi=1)$ or $(R=0)$ is the event horizon and therefore a null hypersurface to all orders.

2. $u^{A}$ is the null generator of this event horizon to all orders.

3. Bulk metric $G_{A B}$ to all orders approaches $g_{A B}$ as $R \rightarrow \infty$.

These conditions translate to the following constraints on the unknown functions.

$$
\begin{gathered}
f_{n}(R=0)=v_{n}(R=0)=0, \quad h_{n}(R=0)=t_{n}(R=0)=\text { finite } \\
\lim _{R \rightarrow \infty} f_{n}(R)=\lim _{R \rightarrow \infty} h_{n}(R)=\lim _{R \rightarrow \infty} v_{n}(R)=\lim _{R \rightarrow \infty} t_{n}(R)=0
\end{gathered}
$$

The homogeneous part $H_{A B}$ (i.e., the part that acts like a differential operator on the space of unknown functions appearing in $G_{A B}^{(2)}$ ) is universal. It will have the same form as in the 'first order' calculation and we do not need to recalculate it. For convenience, here we shall quote the results for the homogeneous part as derived in [1].

$$
\begin{aligned}
H_{A B} \equiv & H^{(1)} O_{A} O_{B}+H^{(2)}\left(n_{A} O_{B}+n_{B} O_{A}\right)+H^{(3)} n_{A} n_{B}+H^{(t r)} P_{A B} \\
& +\left(O_{A} P_{B}^{C}+O_{B} P_{A}^{C}\right) H_{C}^{\left(V_{1}\right)}+\left(n_{A} P_{B}^{C}+n_{B} P_{A}^{C}\right) H_{C}^{\left(V_{2}\right)}+H_{A B}^{(T)}
\end{aligned}
$$


where,

$$
\begin{aligned}
H^{(1)}= & -\frac{N^{2}}{2}\left(1-e^{-R}\right) \sum_{n} \mathfrak{s}_{n}\left(f_{n}^{\prime \prime}+f_{n}^{\prime}\right)-\frac{N}{2} e^{-R} \sum_{n} \frac{\left(\nabla \cdot \mathfrak{v}_{n}\right)}{D} v_{n} \\
& +\frac{N^{2}}{4} e^{-R}\left(1-e^{-R}\right) \sum_{n} \mathfrak{s}_{n} h_{n}^{\prime} \\
H^{(2)}= & \frac{N^{2}}{2} \sum_{n} \mathfrak{s}_{n}\left(f_{n}^{\prime \prime}+f_{n}^{\prime}\right)+\frac{N}{2} \sum_{n} \frac{\left(\nabla \cdot \mathfrak{v}_{n}\right)}{D} v_{n}^{\prime}-\frac{N^{2}}{4} e^{-R} \sum_{n} \mathfrak{s}_{n} h_{n}^{\prime} \\
H^{(3)}= & -\frac{N^{2}}{2} \sum_{n} \mathfrak{s}_{n} h_{n}^{\prime \prime} \\
H^{(t r)}= & 0 \\
H_{C}^{\left(V_{1}\right)}= & -\frac{N^{2}}{2}\left(1-e^{-R}\right) \sum_{n}\left(v_{n}^{\prime \prime}+v_{n}^{\prime}\right)\left[\mathfrak{v}_{n}\right]_{C} \\
H_{C}^{\left(V_{2}\right)}= & \frac{N^{2}}{2} \sum_{n}\left(v_{n}^{\prime \prime}+v_{n}^{\prime}\right)\left[\mathfrak{v}_{n}\right]_{C}+\frac{N}{2 D} \sum_{n} t_{n}^{\prime}\left(\nabla_{D}\left[\mathfrak{t}_{n}\right]_{C}^{D}\right) \\
H_{A B}^{(T)}= & -\frac{N^{2}}{2} \sum_{n}\left[t_{n}^{\prime \prime}\left(1-e^{-R}\right)+t_{n}^{\prime}\right]\left[\mathfrak{t}_{n}\right]_{A B}
\end{aligned}
$$

Here for any $R$ dependent function, $X^{\prime}(R)$ denotes $\frac{d X(R)}{d R}$.

The 'source' parts of these equations are determined by evaluating the Einstein equation on the first order corrected metric. By construction the order $\mathcal{O}\left(D^{2}\right)$ and order $\mathcal{O}(D)$ pieces of these equations will vanish and first non-zero contribution, relevant for the computation of this note, will be of $\mathcal{O}(1)$.

From the above discussion it follows that the key part of the computation is to determine the source term, which we denote here by $S_{A B}$. Since $G_{A B}^{(1)}$ vanishes, just like in [1] here also the source will be given by $E_{A B}$ calculated on $\left(G_{A B}^{(0)}\right)$, however the complication lies in the fact that the calculation has to be carried out upto order $\mathcal{O}(1)$.

Here we are presenting the final result for the source. See appendix A for the details. For convenience, we shall decompose $S_{A B}$ into its different components.

$$
\begin{aligned}
S_{A B} \equiv & S^{(1)} O_{A} O_{B}+S^{(2)}\left(n_{A} O_{B}+n_{B} O_{A}\right)+S^{(3)} n_{A} n_{B}+S^{(t r)} P_{A B} \\
& +\left(O_{A} P_{B}^{C}+O_{B} P_{A}^{C}\right) S_{C}^{\left(V_{1}\right)}+\left(n_{A} P_{B}^{C}+n_{B} P_{A}^{C}\right) S_{C}^{\left(V_{2}\right)}+S_{A B}^{(T)}
\end{aligned}
$$

where $O^{A} S_{A B}^{(T)}=n^{A} S_{A B}^{(T)}=0, \quad S_{A B}^{(T)} P^{A B}=0$ and $P_{A B} \equiv g_{A B}+u_{A} u_{B}-n_{A} n_{B}$ 
The explicit expression for the different components are the following.

$$
\begin{aligned}
S^{(1)}= & e^{-2 R}\left(\frac{K}{2}\right) E^{\text {scalar }}+\left(e^{-R}-e^{-2 R}\right) \mathfrak{s}_{1}+e^{-2 R}\left(\frac{R^{2}}{2}\right)\left(\frac{D}{K}\right)^{2} \mathfrak{s}_{2} \\
& -R\left(\frac{e^{-2 R}}{2}\right)\left(\tilde{\nabla} \cdot E^{\text {vector }}\right)_{R=0} \\
S^{(2)}= & e^{-R}\left[-\mathfrak{s}_{1}+\left(\frac{K}{2}\right) E^{\text {scalar }}\right]_{R=0}-R\left(\frac{e^{-R}}{2}\right)(\tilde{\nabla} \cdot E)_{R=0} \\
& +e^{-R}\left(\frac{R^{2}}{2}\right)\left[\left(\frac{D^{2}}{K^{2}}\right) \mathfrak{s}_{2}\right]_{R=0} \\
S_{C}^{\left(V_{1}\right)}= & \frac{e^{-R}}{2}\left[K E_{C}^{\text {vector }}-2 R\left(\frac{D}{K}\right) \mathfrak{v}_{C}\right], \quad S_{A B}^{(T)}=e^{-R} \mathfrak{t}_{A B} \\
S^{(3)}= & S^{t r}=0, \quad S_{C}^{\left(V_{2}\right)}=0
\end{aligned}
$$

Where

$$
\begin{aligned}
E^{\text {scalar }}= & {\left[\left.(\tilde{\nabla} \cdot u)\right|_{\psi=1}-\frac{1}{2 K}\left[\nabla_{(A} u_{B)} \nabla_{(C} u_{D)} P^{B C} P^{A D}\right]\right] } \\
E_{C}^{\text {vector }}= & {\left[\frac{\tilde{\nabla}^{2} u_{A}}{K}-\frac{\tilde{\nabla}_{A} K}{K}+u^{B} K_{B A}-u \cdot \tilde{\nabla} u_{A}\right] P_{C}^{A} } \\
+ & {\left[-\frac{u^{B} K_{B D} K_{A}^{D}}{K}+\frac{\tilde{\nabla}^{2} \tilde{\nabla}^{2} u_{A}}{K^{3}}-\frac{\left(\tilde{\nabla}_{A} K\right)(u \cdot \tilde{\nabla} K)}{K^{3}}-\frac{\left(\tilde{\nabla}_{B} K\right)\left(\tilde{\nabla}^{B} u_{A}\right)}{K^{2}}\right.} \\
& -\frac{2 K^{D E} \tilde{\nabla}_{D} \tilde{\nabla}_{E} u_{A}}{K^{2}}-\frac{\tilde{\nabla}_{A} \tilde{\nabla}^{2} K}{K^{3}}+\frac{\tilde{\nabla}_{A}\left(K_{B D} K^{B D} K\right)}{K^{3}}+3 \frac{(u \cdot K \cdot u)\left(u \cdot \tilde{\nabla} u_{A}\right)}{K} \\
& -3 \frac{(u \cdot K \cdot u)\left(u^{B} K_{B A}\right)}{K}-6 \frac{(u \cdot \tilde{\nabla} K)\left(u \cdot \tilde{\nabla} u_{A}\right)}{K^{2}}+6 \frac{(u \cdot \tilde{\nabla} K)\left(u^{B} K_{B A}\right)}{K^{2}} \\
& \left.+3 \frac{u \cdot \tilde{\nabla} u_{A}}{D-3}-3 \frac{u^{B} K_{B A}}{D-3}-\frac{(D-1) \lambda}{K^{2}}\left(\frac{\tilde{\nabla}_{A} K}{K}-2 u^{D} K_{D A}+2(u \cdot \tilde{\nabla}) u_{A}\right)\right] P_{C}^{A}
\end{aligned}
$$

See equation (2.13) for the definitions of $\mathfrak{s}_{1}, \mathfrak{s}_{2}, \mathfrak{v}_{C}, \mathfrak{t}_{A B}$.

$\tilde{\nabla}$ is defined as follows: for any general tensor with $n$ indices $W_{A_{1} A_{2} \cdots A_{n}}$

$$
\tilde{\nabla}_{A} W_{A_{1} A_{2} \cdots A_{n}}=\Pi_{A}^{C} \Pi_{A_{1}}^{C_{1}} \Pi_{A_{2}}^{C_{2}} \cdots \Pi_{A_{n}}^{C_{n}}\left(\nabla_{C} W_{C_{1} C_{2} \cdots C_{n}}\right)
$$

The final set of coupled differential equations that we have to solve is simply

$$
H_{A B}+S_{A B}=0
$$

As explained in [1], the homogeneous part $H_{A B}$ could be decoupled after taking its appropriate projection on different directions. Similar projections applied on $S_{A B}$ will generate the sources for the scalar, vector, tensor and the trace sectors.

However, just as in the first order calculation, there is an 'integrability' condition. Note that $H^{(1)}$ and $H_{C}^{\left(V_{1}\right)}$ vanish at $R=0 .{ }^{6}$ Hence consistency demands that $S^{(1)}$ and $S_{C}^{\left(V_{1}\right)}$

\footnotetext{
${ }^{6}$ To see the vanishing of $H^{(1)}$ at $R=0$ we have to use the fact that $v_{n}(R)$ vanishes at $R=0$ as a consequence of our boundary condition. See equation (3.2).
} 
should also vanish on $R=0$. In other words, these set of equations could be consistently solved only if on the horizon the velocity field $u_{A}$ and the extrinsic curvature of the $\psi=1$ membrane (viewed as a hypersurface embedded in the background) together satisfy the following equations.

$$
\begin{aligned}
\left.S^{(1)}\right|_{R=0} & =\left.\left(\frac{K}{2}\right) E^{\text {scalar }}\right|_{R=0}=0 \\
\left.S_{C}^{\left(V_{1}\right)}\right|_{R=0} & =\left.\left(\frac{K}{2}\right) E_{C}^{\text {vector }}\right|_{R=0}=0
\end{aligned}
$$

By appropriate pull-back these equations could be recast as an intrinsic equation on the hypersurface and they generate the next order correction to the constraint equation (2.10). We have described them in equations (2.17).

Once the constraint equations are satisfied, we could see that in the source $S_{A B}$ only two scalar structures $\left(\mathfrak{s}_{1}\right.$ and $\left.\mathfrak{s}_{2}\right)$, one vector structure $\left(\mathfrak{v}_{C}\right)$ and one tensor structure $\left(\mathfrak{t}_{A B}\right)$ appear. So altogether we have 6 unknown functions ( 2 functions for the scalar sector, 2 in the trace sector, 1 in the vector sector and 1 in the tensor sector) into solve for.

The decoupled ODEs for different unknown metric functions:

- Scalar sector:

For $h_{n}(R): \quad H^{(3)}+S^{(3)}=0 \quad$ for $f_{n}(R): \quad H^{(1)}+S^{(1)}=0, \quad n=1,2$

- Vector sector:

For $v(R): H_{C}^{\left(V_{1}\right)}+S_{C}^{\left(V_{1}\right)}=0$

- Tensor sector:

For $t(R): H_{A B}^{(T)}+S_{A B}^{(T)}=0$

Now we shall give the explicit form of the equations sector by sector.

Tensor sector. Here the explicit form of the equation is as follows

$$
t^{\prime \prime}\left(1-e^{-R}\right)+t^{\prime}=\frac{2}{N^{2}} e^{-R}=2\left(\frac{D}{K}\right)^{2} e^{-R}
$$

We can integrate this equation. After imposing

$$
t(R=0)=\text { finite and } \lim _{R \rightarrow \infty} t(R)=0
$$

we find the result as presented in the first equation of (2.15).

Vector sector. Here the explicit form of the equation is as follows

$$
\left(1-e^{-R}\right) \frac{d}{d R}\left(e^{R} v^{\prime}\right)+2\left(\frac{D}{K}\right)^{3} R=0
$$

After imposing

$$
v(R=0)=0 \text { and } \lim _{R \rightarrow \infty} v(R)=0
$$

we find the result as presented in the second equation of (2.15). 
Trace sector. The equations for $h_{n}(R)$ is simply given by

$$
-\frac{N^{2}}{2} \sum_{n} h_{n}^{\prime \prime} \mathfrak{s}_{n}=0
$$

Integrating this differential equation with the boundary condition (3.2), we found correction in the trace sector vanishes i.e., $h_{n}(R)=0$.

Scalar sector. The equations for $f_{1}(R)$ and $f_{2}(R)$ are given by

$$
\begin{aligned}
& e^{-R}\left(1-e^{-R}\right) \frac{d}{d R}\left[e^{R} f_{1}^{\prime}\right]=2\left(\frac{D}{K}\right)^{2} e^{-R}\left(1-e^{-R}\right) \\
& e^{-R}\left(1-e^{-R}\right) \frac{d}{d R}\left[e^{R} f_{2}^{\prime}\right]=-\left(\frac{D}{K}\right) e^{-R} v(R)+\left(\frac{D}{K}\right)^{4} R^{2} e^{-2 R}
\end{aligned}
$$

To derive the second equation we have used the fact (see appendix B.2 for derivation)

$$
\frac{\nabla \cdot \mathfrak{v}}{D}=\mathfrak{s}_{2}
$$

After imposing

$$
f_{n}(R=0)=0 \text { and } \lim _{R \rightarrow \infty} f_{n}(R)=0, \quad n=1,2
$$

we find the result as presented in the third and the fourth equation of (2.15).

\section{Checks}

In this section we shall perform several checks on our calculation. Roughly the checks could be of two types. The first is the internal consistency of our solutions and the systems of equations, i.e, to verify that if we simply substitute our solution in the system of equations (3.10), each and every component of it vanishes upto corrections of order $\mathcal{O}\left(\frac{1}{D}\right)$. The details of it would be presented in subsection 4.1.

The second type of checks are the ones where we shall take several limits and match our results with some answers, known previously. One trivial check in this category that we have tried on every stage of our computation is to match with the known results in asymptotically flat case [4], by setting the cosmological constant $\Lambda$ to zero. The corrected constraint equation (2.17) manifestly matches with equation no (4.5) and (4.12) respectively of [4], if we set $\Lambda$ to zero. At this stage it is difficult to match the two metrics even after setting $\Lambda$ to zero, since our subsidiary conditions are different from that of [4] and we leave it for future.

The other significant check that we have performed is the matching of the spectrum of linearized fluctuation derived from our constraint equations to that of the Quasi-Normal modes already calculated in [16]. In subsection 4.2 we shall give the details of this computation. 


\subsection{Check for internal consistency}

In this subsection we shall explicitly verify that our solution for the metric along with the membrane equations constraining the membrane data, does satisfy equation (3.10) i.e., each of its components vanishes upto corrections of order $\mathcal{O}\left(\frac{1}{D}\right)$.

Let $\mathcal{E}_{A B}$ denote the l.h.s. of equation (3.10).

$$
\mathcal{E}_{A B} \equiv H_{A B}+S_{A B}
$$

From the list of the decoupled ODEs (see the discussion below equation (3.10)) it is clear that the 4 of the 7 independent components of $\mathcal{E}_{A B}$ must be satisfied since we have solved for the metric functions by integrating them. These components are

$$
u^{A} u^{B} \mathcal{E}_{A B}, \quad O^{A} O^{B} \mathcal{E}_{A B}, \quad u^{A} P_{B}^{C} \mathcal{E}_{A C}, \quad P_{A}^{C} P_{B}^{C^{\prime}}\left[\mathcal{E}_{C C^{\prime}}-\left(\frac{\mathcal{E}}{D-2}\right) P_{C C^{\prime}}\right]
$$

where $\mathcal{E}$ denotes the projected trace of $\mathcal{E}_{A B}$ i.e., $\mathcal{E}=P^{A B} \mathcal{E}_{A B}$.

From the explicit expressions of $H_{A B}$ it is clear that $u^{A} H_{A B} u^{B}=H^{(1)}$ and $u^{A} H_{A C} P_{B}^{C}=H_{B}^{\left(V_{1}\right)}$ vanish at $\psi=1$ and membrane equations ensure that the same is true for the source.

As explained in [1], if we consider 'the variation of the metric as we go away from the horizon' as 'dynamics', then the membrane equations play the role of 'constraint equations', whereas the equations we solved to determine the metric corrections are like the 'dynamical' ones. Now in any theory of gravity, it is enough to solve the 'dynamical equations' everywhere and the constraint equation only along one constant 'time slice' (in our case which would be a constant $\psi$ slice); gauge invariance will ensure that the full set of equations are solved everywhere [38]. This theorem guarantees that the rest of the three independent components of $\mathcal{E}_{A B}$ must vanish provided we have solved the equations correctly. These components

$$
\begin{aligned}
& u^{A} O^{B} \mathcal{E}_{A B}=H^{(2)}+S^{(2)} \equiv \mathcal{E}^{(2)} \\
& \frac{1}{D} P^{A B} \mathcal{E}_{A B}=H^{(t r)}+S^{(t r)} \equiv \mathcal{E}^{(t r)} \\
& O^{A} P_{B}^{C} \mathcal{E}_{A C}=H_{B}^{\left(V_{2}\right)}+S_{B}^{\left(V_{2}\right)} \equiv \mathcal{E}_{B}^{\left(V_{2}\right)}
\end{aligned}
$$

Therefore the fact that these components do vanish on our solution is an important consistency check of our whole procedure and the final answer. Computationally it turns out to be quite non-trivial. In fact we have to take help from Mathematica to prove them. 


\subsubsection{Vanishing of $\mathcal{E}^{(2)}$}

From eq (3.4) it follows that

$$
\begin{aligned}
& H^{(2)}=\frac{1}{2}\left(\frac{K}{D}\right)^{2} \sum_{n=1}^{2} \mathfrak{s}_{n}\left(f_{n}^{\prime \prime}+f_{n}^{\prime}-\frac{e^{-R}}{2} h_{n}^{\prime}\right)+\left(\frac{K}{D}\right)\left(\frac{\nabla \cdot \mathfrak{v}}{2 D}\right) v^{\prime} \\
& =\frac{1}{2}\left(\frac{K}{D}\right)^{2} \mathfrak{s}_{1}\left(f_{1}^{\prime \prime}+f_{1}^{\prime}\right)+\frac{1}{2}\left(\frac{K}{D}\right) \mathfrak{s}_{2}\left[N\left(f_{2}^{\prime \prime}+f_{2}^{\prime}\right)+v^{\prime}\right] \\
& =e^{-R_{\mathfrak{s}_{1}}}+\frac{1}{2}\left(\frac{K}{D}\right) \mathfrak{s}_{2}\left[-\frac{e^{-R}}{1-e^{-R}} v+\left(\frac{D}{K}\right)^{3} \frac{R^{2} e^{-R}}{1-e^{-R}}+v^{\prime}\right]
\end{aligned}
$$

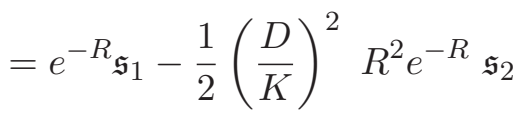

Here we have used the fact that metric correction in the trace sector (i.e., $h_{n}(R)$ ) vanishes. Also we have used equation (3.16) for the divergence of $\mathfrak{v}_{C}$ and the last three equations from (2.15) for the expressions of $f_{n}(R)$ and $v(R)$.

From equation (3.6) we could see that $H^{(2)}$ is exactly the minus of $S^{(2)}$ as required.

\subsubsection{Vanishing of $\mathcal{E}^{(t r)}$}

This follows trivially from (3.6) and (3.4), as both $S^{(t r)}$ and $H^{(t r)}$ vanish at this order.

\subsubsection{Vanishing of $\mathcal{E}_{B}^{\left(V_{2}\right)}$}

From equation (3.6) we see that $S_{C}^{\left(V_{2}\right)}=0$, therefore $H_{C}^{\left(V_{2}\right)}$ should also vanish on our solution. The equation below checks that this is true.

$$
\begin{aligned}
H_{C}^{\left(V_{2}\right)} & \equiv \frac{1}{2}\left(\frac{K}{D}\right)^{2}\left(v^{\prime \prime}+v^{\prime}\right) \mathfrak{v}_{C}+\frac{1}{2}\left(\frac{K}{D}\right) t^{\prime} \frac{\left(\nabla_{D} \mathfrak{t}_{C}^{D}\right)}{D} \\
& =\frac{1}{2}\left(\frac{K}{D}\right)\left[\left(\frac{K}{D}\right)\left(v^{\prime \prime}+v^{\prime}\right)+t^{\prime}\right] \mathfrak{v}_{C} \\
& =0
\end{aligned}
$$

In the second line we have used the identity (see appendix B.1 for the derivation),

$$
\nabla_{D}\left(\mathfrak{t}_{C}^{D}\right)=D \mathfrak{v}_{C}
$$

In the last line we have used the first and the second equation of (2.15) for the expressions of $v(R)$ and $t(R)$.

\subsection{Quasinormal modes for Schwarzschild black hole in background AdS/dS spacetime}

Now as a check for our membrane equations, we will calculate the light quasinormal mode frequencies for Schwarzschild black hole in AdS/dS background. As expected, we find that the answers for the frequencies of light quasinormal modes match exactly with those derived in [16] from gravitational analysis. 
As before, we shall follow [1] for the computation. Many steps and arguments are exactly same as in [1]. For such steps we shall simply refer to [1] or quote them in the appendix. And here we shall present only those parts of computation where we have to do some extension of what has been done in [1].

We shall write the background AdS/dS in global coordinates as

$$
d s_{(b g d)}^{2}=g_{A B} d X^{A} d X^{B}=-\left(1-\sigma \frac{r^{2}}{L^{2}}\right) d t^{2}+\frac{d r^{2}}{\left(1-\sigma \frac{r^{2}}{L^{2}}\right)}+r^{2} d \Omega_{D-2}^{2} .
$$

Where

$$
\begin{array}{rlrl}
\Lambda & =\frac{\sigma}{L^{2}}(D-1)(D-2) \\
L & =\text { AdS } / \mathrm{dS} \text { radius } \\
\sigma & =0 & & \text { for Flat } \\
& =1 & & \text { for } \mathrm{dS} \\
& =-1 & & \text { for AdS }
\end{array}
$$

And the Schwarzschild black hole in this coordinate system is

$$
d s_{(B H)}^{2}=-\left(1-\sigma \frac{r^{2}}{L^{2}}-\left(\frac{r_{0}}{r}\right)^{D-3}\right) d t^{2}+\frac{d r^{2}}{\left(1-\sigma \frac{r^{2}}{L^{2}}-\left(\frac{r_{0}}{r}\right)^{D-3}\right)}+r^{2} d \Omega_{D-2}^{2} .
$$

Where, $r_{0}$ is an arbitrary constant. Note that the position of horizon is $r=r_{H}$ where $r_{H}$ is the zero of the function $f(r)=\left(1-\sigma \frac{r^{2}}{L^{2}}-\left(\frac{r_{0}}{r}\right)^{D-3}\right)$.

$$
r_{H}=r_{0}\left(1-\frac{1}{D} \ln \left(1-\frac{\sigma r_{0}^{2}}{L^{2}}\right)+\mathcal{O}\left(D^{-2}\right)\right)
$$

From now on we choose $r_{H}=1$ or in other words $r_{0}$ will be set to

$$
r_{0}=\left(1+\frac{1}{D} \ln \left(1-\frac{\sigma}{L^{2}}\right)+\mathcal{O}\left(D^{-2}\right)\right)
$$

for convenience. We will later reinstate the factors of $r_{0}$ from dimensional analysis.

A small fluctuation around a static black hole corresponds to a small fluctuation around a spherical membrane along with a small fluctuation in the velocity field, which is purely in the time direction at zeroth order. We will work upto linear order in the amplitude of fluctuations, which we denote by $\epsilon$.

$$
\begin{aligned}
& r=1+\epsilon \delta r(t, a) \\
& u=u_{0} d t+\epsilon \delta u_{\mu}(t, a) d x^{\mu}
\end{aligned}
$$

Here, we denote the angle coordinates along $(D-2)$ dimensional sphere by $a$ and the coordinates $\mu$ on the membrane worldvolume contain time $t$ and angles $a$. The induced metric on the membrane worldvolume (viewed as a hypersurface embedded in the background metric (4.4)) upto linear order in $\epsilon$ is (where we denote the metric components by $g_{\mu \nu}^{(\text {ind) }}$ )

$$
d s_{(\text {ind })}^{2}=g_{\mu \nu}^{(\text {ind })} d y^{\mu} d y^{\nu}=-\left(1-\sigma \frac{1+2 \epsilon \delta r}{L^{2}}\right) d t^{2}+(1+2 \epsilon \delta r) d \Omega_{D-2}^{2}
$$


Also, $u_{\mu} g_{\text {(ind) }}^{\mu \nu} u_{\nu}=-1$ implies

$$
u_{0}=-\left(1-\frac{\sigma}{L^{2}}\right)^{\frac{1}{2}} \text { and } \delta u_{t}(t, a)=\left(1-\frac{\sigma}{L^{2}}\right)^{-\frac{1}{2}}\left(\frac{\sigma}{L^{2}}\right) \delta r(t, a)
$$

The membrane equations are

$$
\begin{aligned}
\hat{\nabla} \cdot u= & \frac{1}{2 \mathcal{K}}\left(\hat{\nabla}_{(\alpha} u_{\beta)} \hat{\nabla}_{(\gamma} u_{\delta)} \mathcal{P}^{\beta \gamma} \mathcal{P}^{\alpha \delta}\right) \\
E_{\mu}^{\mathrm{tot}} \equiv & {\left[\frac{\hat{\nabla}^{2} u_{\alpha}}{\mathcal{K}}-\frac{\hat{\nabla}_{\alpha} \mathcal{K}}{\mathcal{K}}+u^{\beta} \mathcal{K}_{\beta \alpha}-u \cdot \hat{\nabla} u_{\alpha}\right] \mathcal{P}_{\gamma}^{\alpha}+\left[-\frac{u^{\beta} \mathcal{K}_{\beta \delta} \mathcal{K}_{\alpha}^{\delta}}{\mathcal{K}}+\frac{\hat{\nabla}^{2} \hat{\nabla}^{2} u_{\alpha}}{\mathcal{K}^{3}}\right.} \\
& -\frac{\left(\hat{\nabla}_{\alpha} \mathcal{K}\right)(u \cdot \hat{\nabla} \mathcal{K})}{\mathcal{K}^{3}}-\frac{\left(\hat{\nabla}_{\beta} \mathcal{K}\right)\left(\hat{\nabla}^{\beta} u_{\alpha}\right)}{\mathcal{K}^{2}}-\frac{2 \mathcal{K}^{\delta \sigma} \hat{\nabla}_{\delta} \hat{\nabla}_{\sigma} u_{\alpha}}{K^{2}}-\frac{\hat{\nabla}_{\alpha} \hat{\nabla}^{2} \mathcal{K}}{\mathcal{K}^{3}}+\frac{\hat{\nabla}_{\alpha}\left(\mathcal{K}_{\beta \delta} \mathcal{K}^{\beta \delta} \mathcal{K}\right)}{K^{3}} \\
& +3 \frac{(u \cdot \mathcal{K} \cdot u)\left(u \cdot \hat{\nabla} u_{\alpha}\right)}{\mathcal{K}}-3 \frac{(u \cdot \mathcal{K} \cdot u)\left(u^{\beta} \mathcal{K}_{\beta \alpha}\right)}{\mathcal{K}}-6 \frac{(u \cdot \hat{\nabla} \mathcal{K})\left(u \cdot \hat{\nabla} u_{\alpha}\right)}{\mathcal{K}^{2}}+6 \frac{(u \cdot \hat{\nabla} \mathcal{K})\left(u^{\beta} \mathcal{K}_{\beta \alpha}\right)}{\mathcal{K}^{2}} \\
& \left.+3 \frac{u \cdot \hat{\nabla} u_{\alpha}}{D-3}-3 \frac{u^{\beta} \mathcal{K}_{\beta \alpha}}{D-3}-\frac{(D-1) \lambda}{\mathcal{K}^{2}}\left(\frac{\hat{\nabla}_{\alpha} \mathcal{K}}{\mathcal{K}}-2 u^{\sigma} \mathcal{K}_{\sigma \alpha}+2(u \cdot \hat{\nabla}) u_{\alpha}\right)\right] \mathcal{P}_{\gamma}^{\alpha}=0
\end{aligned}
$$

In (4.11) and (4.12), the covariant derivative with respect to metric (4.9) is denoted by $\hat{\nabla}$. The extrinsic curvature of membrane is denoted by $\mathcal{K}_{\mu \nu}$ and its trace by $\mathcal{K}$. The projector orthogonal to $u_{\mu}$ is denoted by $\mathcal{P}_{\mu \nu}$.

It turns out that $E_{t}^{\text {tot }}$ vanishes at linear order in $\epsilon$. Using (C.14) and (C.15), we evaluate the vector membrane equation in the angular directions

$$
\begin{aligned}
E_{a}^{\mathrm{tot}} \equiv & {\left[(D-2)-\left(1-\frac{\sigma}{L^{2}}\right)^{-1} \frac{\sigma}{L^{2}}\right]^{-1}\left[-\left(1-\frac{\sigma}{L^{2}}\right)^{-\frac{1}{2}}\left(\epsilon \partial_{t}^{2} \delta u_{a}\right)+\left(1-\frac{\sigma}{L^{2}}\right)^{-1} \frac{\sigma}{L^{2}}\left(\epsilon \partial_{t} \bar{\nabla}_{a} \delta r\right)\right.} \\
& \left.+\left(1-\frac{\sigma}{L^{2}}\right)^{\frac{1}{2}} \epsilon \bar{\nabla}^{2} \delta u_{a}+\epsilon \partial_{t} \bar{\nabla}_{a} \delta r\right]-\left[(D-2)-\left(1-\frac{\sigma}{L^{2}}\right)^{-1} \frac{\sigma}{L^{2}}\right]^{-1} \times \\
& \times\left[\left(1-\frac{\sigma}{L^{2}}\right)^{-1}\left(\epsilon \partial_{t}^{2} \bar{\nabla}_{a} \delta r\right)-\left(1-\frac{\sigma}{L^{2}}\right)^{-1} \frac{\sigma}{L^{2}}\left(\epsilon \bar{\nabla}_{a} \delta r\right)-\epsilon \bar{\nabla}_{a} \bar{\nabla}^{2} \delta r-(D-2)\left(\epsilon \bar{\nabla}_{a} \delta r\right)\right] \\
& +\left[\left(1-\frac{\sigma}{L^{2}}\right)^{-1}\left(-\epsilon \partial_{t} \bar{\nabla}_{a} \delta r\right)+\left(1-\frac{\sigma}{L^{2}}\right)^{\frac{1}{2}}\left(\epsilon \delta u_{a}\right)+\left(1-\frac{\sigma}{L^{2}}\right)^{-\frac{1}{2}} \frac{\sigma}{L^{2}} \delta u_{a}\right] \\
& -\left[\left(1-\frac{\sigma}{L^{2}}\right)^{-\frac{1}{2}}\left(\epsilon \partial_{t} \delta u_{a}\right)-\left(1-\frac{\sigma}{L^{2}}\right)^{-1} \frac{\sigma}{L^{2}}\left(\epsilon \bar{\nabla}_{a} \delta r\right)\right] \\
& -\frac{1}{D-2}\left[\left(1-\frac{\sigma}{L^{2}}\right)^{-2} \frac{\sigma}{L^{2}} \epsilon \partial_{t} \bar{\nabla}_{a} \delta r-\left(1-\frac{\sigma}{L^{2}}\right)^{-1} \epsilon \partial_{t} \bar{\nabla}_{a} \delta r+\left(1-\frac{\sigma}{L^{2}}\right)^{\frac{1}{2}} \epsilon \delta u_{a}\right. \\
& \left.-\left(\frac{\sigma}{L^{2}}\right)^{2}\left(1-\frac{\sigma}{L^{2}}\right)^{-\frac{3}{2}} \delta u_{a}\right]+\frac{1}{(D-2)^{3}}\left(1-\frac{\sigma}{L^{2}}\right)^{-\frac{3}{2}}\left[\bar{\nabla}^{2} \bar{\nabla}^{2} \delta u_{a}\right] \\
& -\frac{2}{(D-2)^{2}}\left(1-\frac{\sigma}{L^{2}}\right)^{-\frac{1}{2}} \epsilon \hat{\nabla}^{2} \delta u_{a}-\frac{1}{(D-2)^{3}}\left(1-\frac{\sigma}{L^{2}}\right)^{-2}\left[-\hat{\nabla}_{a} \hat{\nabla}^{2} \hat{\nabla}^{2} \delta r-(D-2) \hat{\nabla}_{a} \hat{\nabla}^{2} \delta r\right] \\
& +\frac{1}{(D-2)^{3}}\left(1-\frac{\sigma}{L^{2}}\right)^{-1}\left[-3(D-2) \epsilon\left(\hat{\nabla}_{a} \hat{\nabla}^{2} \delta r+(D-2) \hat{\nabla}_{a} \delta r\right)\right] \\
& +\frac{3}{D-2}\left[\left(1-\frac{\sigma}{L^{2}}\right)^{-\frac{3}{2}} \frac{\sigma}{L^{2}} \epsilon \partial_{t} \delta u_{a}-\left(1-\frac{\sigma}{L^{2}}\right)^{-2}\left(\frac{\sigma}{L^{2}}\right)^{2} \epsilon \hat{\nabla}_{a} \delta r\right]
\end{aligned}
$$




$$
\begin{aligned}
& -\frac{3}{D-2}\left(1-\frac{\sigma}{L^{2}}\right)^{-\frac{1}{2}}\left[-\left(1-\frac{\sigma}{L^{2}}\right)^{-\frac{3}{2}}\left(\frac{\sigma}{L^{2}}\right) \epsilon \partial_{t} \hat{\nabla}_{a} \delta r+\frac{\sigma}{L^{2}} \epsilon \delta u_{a}+\left(\frac{\sigma}{L^{2}}\right)^{2}\left(1-\frac{\sigma}{L^{2}}\right)^{-1} \delta u_{a}\right] \\
& +\frac{3}{D-2}\left[\left(1-\frac{\sigma}{L^{2}}\right)^{-\frac{1}{2}}\left(\epsilon \partial_{t} \delta u_{a}\right)-\left(1-\frac{\sigma}{L^{2}}\right)^{-1} \frac{\sigma}{L^{2}}\left(\epsilon \bar{\nabla}_{a} \delta r\right)\right] \\
& -\frac{3}{D-2}\left[\left(1-\frac{\sigma}{L^{2}}\right)^{-1}\left(-\epsilon \partial_{t} \bar{\nabla}_{a} \delta r\right)+\left(1-\frac{\sigma}{L^{2}}\right)^{\frac{1}{2}}\left(\epsilon \delta u_{a}\right)+\frac{\sigma}{L^{2}}\left(1-\frac{\sigma}{L^{2}}\right)^{-\frac{1}{2}} \delta u_{a}\right] \\
& -\frac{2}{D-2} \frac{\sigma}{L^{2}}\left[\left(1-\frac{\sigma}{L^{2}}\right)^{-\frac{3}{2}}\left(\epsilon \partial_{t} \delta u_{a}\right)-\left(1-\frac{\sigma}{L^{2}}\right)^{-2} \frac{\sigma}{L^{2}}\left(\epsilon \bar{\nabla}_{a} \delta r\right)\right] \\
& +\frac{2}{D-2} \frac{\sigma}{L^{2}}\left[\left(1-\frac{\sigma}{L^{2}}\right)^{-2}\left(-\epsilon \partial_{t} \bar{\nabla}_{a} \delta r\right)+\left(1-\frac{\sigma}{L^{2}}\right)^{-\frac{1}{2}}\left(\epsilon \delta u_{a}\right)+\frac{\sigma}{L^{2}}\left(1-\frac{\sigma}{L^{2}}\right)^{-\frac{3}{2}} \delta u_{a}\right] \\
& -\frac{1}{D-2} \frac{\sigma}{L^{2}}\left(1-\frac{\sigma}{L^{2}}\right)^{-2}\left[\frac{1}{D-2}\left(-\epsilon \bar{\nabla}_{a} \bar{\nabla}^{2} \delta r-(D-2) \epsilon \bar{\nabla}_{a} \delta r\right)\right]
\end{aligned}
$$

Where, in (4.13) we have neglected the terms which are order $\mathcal{O}\left(1 / D^{2}\right)$ or higher. We denote the covariant derivative with respect to a unit sphere metric in $D-2$ dimensions by $\bar{\nabla}_{a}$. Similarly we evaluate the membrane equation (4.11)

$$
\hat{\nabla} \cdot u=\epsilon \bar{\nabla}^{a} \delta u_{a}+\epsilon\left(1-\frac{\sigma}{L^{2}}\right)^{-\frac{1}{2}}\left(\partial_{t} \delta r\right)(D-2)=0
$$

We choose to divide the fluctuation $\delta u_{a}$ in two parts (see section (5) of [3])

$$
\delta u_{a}=\delta v_{a}+\bar{\nabla}_{a} \Phi, \quad \text { with } \quad \bar{\nabla}^{a} \delta v_{a}=0
$$

Substituting (4.15) into (4.14) we find

$$
\bar{\nabla}^{2} \Phi=-(D-2)\left(1-\frac{\sigma}{L^{2}}\right)^{-\frac{1}{2}}\left(\partial_{t} \delta r\right)
$$

Now we evaluate $\bar{\nabla}^{a} E_{a}^{\text {tot }}$. We use the identity $\bar{\nabla}^{a} \bar{\nabla}^{2} V_{a}=\left((D-3)+\bar{\nabla}^{2}\right) \bar{\nabla}^{a} V_{a}$ for simplification. We find

$$
\begin{aligned}
\bar{\nabla}^{a} E_{a}^{\mathrm{tot}} \equiv & {\left[(D-2)-\left(1-\frac{\sigma}{L^{2}}\right)^{-1} \frac{\sigma}{L^{2}}\right]^{-1}\left[(D-2)\left(1-\frac{\sigma}{L^{2}}\right)^{-1}\left(\epsilon \partial_{t}^{3} \delta r\right)\right.} \\
& \left.+\left(1-\frac{\sigma}{L^{2}}\right)^{-1} \frac{\sigma}{L^{2}}\left(\epsilon \partial_{t} \bar{\nabla}^{2} \delta r\right)-(D-2) \epsilon \partial_{t}\left(D-3+\bar{\nabla}^{2}\right) \delta r+\left(\epsilon \partial_{t} \bar{\nabla}^{2} \delta r\right)\right] \\
& -\left[(D-2)-\left(1-\frac{\sigma}{L^{2}}\right)^{-1} \frac{\sigma}{L^{2}}\right]^{-1}\left[\left(1-\frac{\sigma}{L^{2}}\right)^{-1}\left(\epsilon \partial_{t}^{2} \bar{\nabla}^{2} \delta r\right)-\left(1-\frac{\sigma}{L^{2}}\right)^{-1} \frac{\sigma}{L^{2}} \epsilon \bar{\nabla}^{2} \delta r\right. \\
& \left.-\epsilon \bar{\nabla}^{2} \bar{\nabla}^{2} \delta r-(D-2) \epsilon \bar{\nabla}^{2} \delta r\right]+\frac{2}{(D-2)}\left(1-\frac{\sigma}{L^{2}}\right)^{-1}\left[\epsilon\left(D-3+\hat{\nabla}^{2}\right) \partial_{t} \delta r\right] \\
& +\left[\left(1-\frac{\sigma}{L^{2}}\right)^{-1}\left(-\epsilon \partial_{t} \bar{\nabla}^{2} \delta r\right)-(D-2) \epsilon \partial_{t} \delta r-(D-2)\left(1-\frac{\sigma}{L^{2}}\right)^{-1} \frac{\sigma}{L^{2}} \partial_{t} \delta r\right] \\
& -\left[-(D-2)\left(1-\frac{\sigma}{L^{2}}\right)^{-1}\left(\epsilon \partial_{t}^{2} \delta r\right)-\left(1-\frac{\sigma}{L^{2}}\right)^{-1} \frac{\sigma}{L^{2}}\left(\epsilon \bar{\nabla}^{2} \delta r\right)\right] \\
& -\frac{1}{D-2}\left[\left(1-\frac{\sigma}{L^{2}}\right)^{-2} \frac{\sigma}{L^{2}} \epsilon \partial_{t} \bar{\nabla}^{2} \delta r-\left(1-\frac{\sigma}{L^{2}}\right)^{-1} \epsilon \partial_{t} \bar{\nabla}^{2} \delta r-(D-2) \epsilon \partial_{t} \delta r\right.
\end{aligned}
$$




$$
\begin{aligned}
& \left.+(D-2)\left(\frac{\sigma}{L^{2}}\right)^{2}\left(1-\frac{\sigma}{L^{2}}\right)^{-2} \partial_{t} \delta r\right] \\
& -\frac{1}{(D-2)^{2}}\left(1-\frac{\sigma}{L^{2}}\right)^{-2} \epsilon\left[\left(D-3+\bar{\nabla}^{2}\right)\left(D-3+\bar{\nabla}^{2}\right) \partial_{t} \delta r\right] \\
& -\frac{1}{(D-2)^{3}}\left(1-\frac{\sigma}{L^{2}}\right)^{-2}\left[-\hat{\nabla}^{2} \hat{\nabla}^{2} \hat{\nabla}^{2} \delta r-(D-2) \hat{\nabla}^{2} \hat{\nabla}^{2} \delta r\right] \\
& -\frac{1}{(D-2)^{2}}\left(1-\frac{\sigma}{L^{2}}\right)^{-1}\left[3 \epsilon\left(\hat{\nabla}^{2} \hat{\nabla}^{2} \delta r+(D-2) \hat{\nabla}^{2} \delta r\right)\right] \\
& +\frac{3}{D-2}\left(1-\frac{\sigma}{L^{2}}\right)^{-2}\left[-(D-2) \frac{\sigma}{L^{2}} \epsilon \partial_{t}^{2} \delta r-\left(\frac{\sigma}{L^{2}}\right)^{2} \epsilon \hat{\nabla}^{2} \delta r\right] \\
& -\frac{3}{D-2}\left[-\left(1-\frac{\sigma}{L^{2}}\right)^{-2}\left(\frac{\sigma}{L^{2}}\right) \epsilon \partial_{t} \hat{\nabla}^{2} \delta r-(D-2) \frac{\sigma}{L^{2}}\left(1-\frac{\sigma}{L^{2}}\right)^{-1} \epsilon \partial_{t} \delta r\right. \\
& \left.-(D-2)\left(\frac{\sigma}{L^{2}}\right)^{2}\left(1-\frac{\sigma}{L^{2}}\right)^{-2} \partial_{t} \delta r\right]+\frac{3}{D-2}\left(1-\frac{\sigma}{L^{2}}\right)^{-1}\left[-(D-2)\left(\epsilon \partial_{t}^{2} \delta r\right)-\frac{\sigma}{L^{2}}\left(\epsilon \bar{\nabla}^{2} \delta r\right)\right] \\
& -\frac{3}{D-2}\left[\left(1-\frac{\sigma}{L^{2}}\right)^{-1}\left(-\epsilon \partial_{t} \bar{\nabla}^{2} \delta r\right)-(D-2)\left(\epsilon \partial_{t} \delta r\right)-(D-2) \frac{\sigma}{L^{2}}\left(1-\frac{\sigma}{L^{2}}\right)^{-1} \partial_{t} \delta r\right] \\
& -\frac{2}{D-2} \frac{\sigma}{L^{2}}\left(1-\frac{\sigma}{L^{2}}\right)^{-2}\left[-(D-2)\left(\epsilon \partial_{t}^{2} \delta r\right)-\frac{\sigma}{L^{2}}\left(\epsilon \bar{\nabla}^{2} \delta r\right)\right] \\
& +\frac{2}{D-2} \frac{\sigma}{L^{2}}\left(1-\frac{\sigma}{L^{2}}\right)^{-2}\left[\left(-\epsilon \partial_{t} \bar{\nabla}^{2} \delta r\right)-(D-2)\left(1-\frac{\sigma}{L^{2}}\right)^{1}\left(\epsilon \partial_{t} \delta r\right)-(D-2) \frac{\sigma}{L^{2}} \partial_{t} \delta r\right] \\
& -\frac{1}{D-2} \frac{\sigma}{L^{2}}\left(1-\frac{\sigma}{L^{2}}\right)^{-2}\left[\frac{1}{D-2}\left(\left(-\epsilon \bar{\nabla}^{2} \bar{\nabla}^{2} \delta r\right)-(D-2)\left(\epsilon \bar{\nabla}^{2} \delta r\right)\right)\right]
\end{aligned}
$$

Now we reinstate the factors of $r_{H} \cdot{ }^{7}$ We expand the shape fluctuations

$$
\delta r=\sum_{l, m} a_{l m} Y_{l m} e^{-i \omega_{l}^{s} r_{H} t}
$$

where, $Y_{l m}$ are the scalar spherical harmonics on $S^{D-2}$ for which

$$
\bar{\nabla}^{2} Y_{l m}=-l(D+l-3) Y_{l m} .
$$

Now, we substitute (4.18) in (4.17) and solve for the scalar QNM frequencies

$$
\begin{aligned}
\omega^{s} r_{0}= & \pm \sqrt{l\left(1-\frac{\sigma r_{0}^{2}}{L^{2}}\right)-1}\left[1+\frac{1}{2 D} \frac{l-1}{l-\left(1-\frac{\sigma r_{0}^{2}}{L^{2}}\right)^{-1}}\left(\left(2\left(1-\frac{\sigma r_{0}^{2}}{L^{2}}\right)^{-1}+1\right) l\right.\right. \\
& \left.\left.-4\left(1-\frac{\sigma r_{0}^{2}}{L^{2}}\right)^{-1}+2\left(1-\frac{\sigma r_{0}^{2}}{L^{2}}\right)^{-1} \ln \left(1-\frac{\sigma r_{0}^{2}}{L^{2}}\right)\right)\right] \\
- & i(l-1)\left[1+\frac{1}{D}\left(l-2+\ln \left(1-\frac{\sigma r_{0}^{2}}{L^{2}}\right)\right)\right]
\end{aligned}
$$

Upto the required order, the answer (4.20) agrees with the respective answer given in equations (D.3), (D.4) of [16].

\footnotetext{
${ }^{7}$ We use the dimensional analysis to replace $L$ by $\frac{L}{r_{H}}$ and replace $\omega^{s}$ by $\omega^{s} r_{H}$. Where, $r_{H}$ is defined in terms of $r_{0}$ in (4.7).
} 
Similarly we now calculate the vector QNM frequencies. Note that we have solved (4.17). So, the $\delta r$ and $\Phi$ terms in (4.13) will drop out and we have

$$
\begin{aligned}
E_{a}^{\mathrm{tot}} \equiv & {\left[(D-2)\left(1-\frac{\sigma}{L^{2}}\right)^{\frac{1}{2}}-\left(1-\frac{\sigma}{L^{2}}\right)^{\frac{-1}{2}} \frac{\sigma}{L^{2}}\right]^{-1}\left[-\left(1-\frac{\sigma}{L^{2}}\right)^{-1}\left(\epsilon \partial_{t}^{2} \delta v_{a}\right)+\epsilon \bar{\nabla}^{2} \delta v_{a}\right] } \\
& +\left[\left(1-\frac{\sigma}{L^{2}}\right)^{\frac{1}{2}}\left(\epsilon \delta v_{a}\right)+\left(1-\frac{\sigma}{L^{2}}\right)^{-\frac{1}{2}} \frac{\sigma}{L^{2}} \delta v_{a}\right]-\left[\left(1-\frac{\sigma}{L^{2}}\right)^{-\frac{1}{2}}\left(\epsilon \partial_{t} \delta v_{a}\right)\right] \\
& -\frac{1}{D-2}\left(1-\frac{\sigma}{L^{2}}\right)^{-\frac{1}{2}}\left[\left(1-\frac{\sigma}{L^{2}}\right) \epsilon \delta v_{a}-\left(\frac{\sigma}{L^{2}}\right)^{2}\left(1-\frac{\sigma}{L^{2}}\right)^{-1} \delta v_{a}\right] \\
& +\frac{1}{(D-2)^{3}}\left(1-\frac{\sigma}{L^{2}}\right)^{-\frac{3}{2}}\left[\bar{\nabla}^{2} \bar{\nabla}^{2} \delta v_{a}\right]-\frac{2}{(D-2)^{2}}\left(1-\frac{\sigma}{L^{2}}\right)^{-\frac{1}{2}}\left[\epsilon \hat{\nabla}^{2} \delta v_{a}\right] \\
& +\frac{3}{D-2}\left(1-\frac{\sigma}{L^{2}}\right)^{-\frac{3}{2}}\left[\frac{\sigma}{L^{2}} \epsilon \partial_{t} \delta v_{a}\right]+\frac{3}{D-2}\left[\left(1-\frac{\sigma}{L^{2}}\right)^{-\frac{1}{2}}\left(\epsilon \partial_{t} \delta v_{a}\right)\right] \\
& -\frac{3}{D-2}\left(1-\frac{\sigma}{L^{2}}\right)^{-\frac{1}{2}}\left[\frac{\sigma}{L^{2}} \epsilon \delta v_{a}+\left(\frac{\sigma}{L^{2}}\right)^{2}\left(1-\frac{\sigma}{L^{2}}\right)^{-1} \delta v_{a}\right] \\
& -\frac{3}{D-2}\left[\left(1-\frac{\sigma}{L^{2}}\right)^{\frac{1}{2}}\left(\epsilon \delta v_{a}\right)+\frac{\sigma}{L^{2}}\left(1-\frac{\sigma}{L^{2}}\right)^{-\frac{1}{2}} \delta v_{a}\right] \\
& -\frac{2}{D-2} \frac{\sigma}{L^{2}}\left[\left(1-\frac{\sigma}{L^{2}}\right)^{-\frac{3}{2}}\left(\epsilon \partial_{t} \delta v_{a}\right)\right] \\
& +\frac{2}{D-2} \frac{\sigma}{L^{2}}\left[\left(1-\frac{\sigma}{L^{2}}\right)^{-\frac{1}{2}}\left(\epsilon \delta v_{a}\right)+\frac{\sigma}{L^{2}}\left(1-\frac{\sigma}{L^{2}}\right)^{-\frac{3}{2}} \delta v_{a}\right]
\end{aligned}
$$

We expand the $\delta v_{a}$ fluctuations as

$$
\delta v_{a}=\sum_{l, m} b_{l m} Y_{a}^{l m} e^{-i \omega_{l}^{v} r_{H} t}
$$

where, $Y_{a}^{l m}$ are the vector spherical harmonics on $S^{D-2}$ for which

$$
\bar{\nabla}^{2} Y_{a}^{l m}=-[(D+l-3) l-1] Y_{a}^{l m}
$$

We Substitute (4.22) in (4.21) and solve for vector QNM frequencies

$$
\omega^{v} r_{0}=-i(l-1)\left[1+\frac{1}{D}\left(l-1+\ln \left(1-\frac{\sigma r_{0}^{2}}{L^{2}}\right)\right)\right]
$$

Upto the required order, the answer (4.24) agrees with the respective answer given in equation (D.2) of [16].

\subsection{Quasinormal modes for AdS Schwarzschild black brane}

Now we shall repeat the above analysis for the case of uniform planar membrane in AdS. This membrane corresponds to AdS Schwarzschild black brane with horizon topology of $R^{D-2} \times R$ in Poincare patch metric. Here we consider membrane fluctuations in time and all the $D-2$ spatial brane directions.

The background metric in Poincare patch coordinates is

$$
d s^{2}=-\hat{r}^{2} d \hat{t}^{2}+\frac{d \hat{r}^{2}}{\hat{r}^{2}}+\hat{r}^{2} d \hat{x}^{a} d \hat{x}_{a}
$$


Where we have set AdS radius $L=1$, i.e. $\Lambda=(D-1)(D-2)$. For our convenience we use the following notation for this section

$$
n \equiv D-1
$$

We consider a uniform planar membrane located at the location $\hat{r}=r_{0}$. We find it convenient to perform the following rescaling

$$
\hat{r}=r_{0} r, \quad \hat{t}=\frac{t}{r_{0}}, \quad \hat{x}^{a}=\frac{x^{a}}{r_{0}}
$$

With this rescaling, the background metric (4.25) becomes

$$
d s_{(b g d)}^{2}=g_{A B} d X^{A} d X^{B}=-r^{2} d t^{2}+\frac{d r^{2}}{r^{2}}+r^{2} d x^{a} d x_{a}
$$

Where now $r=1$ is the location of the uniform membrane. We will consider the time dependence of the shape and velocity fluctuations of the form

$$
e^{-i \hat{\omega} \hat{t}}=e^{-i \omega t}, \quad \text { where } \hat{\omega}=\omega r_{0}
$$

This choice means that the new coordinates in (4.28) are all dimensionless.

We consider the fluctuations around the uniform planar membrane as

$$
\begin{aligned}
& r=1+\epsilon \delta r(t, a) \\
& u=u_{0} d t+\epsilon \delta u_{t}(t, a) d t+\epsilon \delta u_{b}(t, a) d x^{b}
\end{aligned}
$$

Where $\epsilon$ is the amplitude of fluctuations and we work upto linear order in $\epsilon$.

Upto linear order, the induced metric on the membrane worldvolume becomes

$$
d s^{2}=g_{\mu \nu}^{(\mathrm{ind})} d y^{\mu} d y^{\nu}=-(1+2 \epsilon \delta r) d t^{2}+(1+2 \epsilon \delta r) d x^{a} d x_{a}
$$

Upto linear order, $u_{\mu} g_{\text {(ind) }}^{\mu \nu} u_{\nu}=-1$ implies

$$
u_{t}=u_{0}+\epsilon \delta u_{t}=-(1+\epsilon \delta r)
$$

The covariant derivative with respect to induced metric (4.30) is denoted by $\hat{\nabla}$ and of the background metric (4.28) is denoted by $\nabla$. Also $\mathcal{K}_{\mu \nu}$ and $\mathcal{K}$ are defined in the same way as the previous subsection. So we now again consider the membrane equations (4.11) and (4.12).

Substituting the equations (4.29) and (4.31) in the 1.h.s. (4.12) (see appendix (D) for details) we find that $E_{t}^{\text {tot }}$ is of order $\mathcal{O}\left(\epsilon^{2}\right)$, and the ' $a$ ' components of the equation becomes

$$
\begin{aligned}
E_{a}^{\mathrm{tot}}= & \epsilon\left[-\partial_{t} \delta u_{a}-\frac{\partial_{t}^{2} \delta u_{a}}{n}+\frac{\partial^{2} \delta u_{a}}{n}+\frac{\partial_{t}^{4} \delta u_{a}}{n^{3}}-2 \frac{\partial_{t}^{2} \partial^{2} \delta u_{a}}{n^{3}}+\frac{\partial^{2} \partial^{2} \delta u_{a}}{n^{3}}+2 \frac{\partial_{t}^{2} \delta u_{a}}{n^{2}}\right. \\
& \left.-2 \frac{\partial^{2} \delta u_{a}}{n^{2}}+2 \frac{\partial_{t} \delta u_{a}}{n}\right]+\left[-\partial_{a} \delta r-\partial_{t} \partial_{a} \delta r-\frac{\partial_{a} \partial_{t}^{2} \delta r}{n}+\frac{\partial^{2} \partial_{a} \delta r}{n}+2 \frac{\partial_{a} \partial_{t} \delta r}{n}\right. \\
& +\frac{\partial_{a} \partial_{t}^{4} \delta r-2 \partial_{t}^{2} \partial_{a} \partial^{2} \delta r+\partial_{a} \partial^{2} \partial^{2} \delta r}{n^{3}}+3 \frac{\partial_{a} \partial_{t}^{2} \delta r-\partial_{a} \partial^{2} \delta r}{n^{2}}+\frac{\partial_{a} \partial_{t}^{2} \delta r}{n^{2}}-\frac{\partial_{a} \partial^{2} \delta r}{n^{2}} \\
& \left.+2 \frac{\partial_{a} \partial_{t} \delta r}{n}+2 \frac{\partial_{a} \delta r}{n}\right]=0
\end{aligned}
$$


Similarly the expansion of equation (4.11) to linear order in fluctuation leads to the following equation

$$
\hat{\nabla} \cdot u=0=\epsilon \partial^{a} \delta u_{a}+\epsilon(n-1) \partial_{t} \delta r
$$

Now to find the scalar QNM frequencies, the relevant equations are (4.33) and $\partial^{a} E_{a}^{\text {tot }}$. Finding $\partial^{a} E_{a}^{\text {tot }}$ and substituting (4.33) we get

$$
\begin{array}{r}
-(n-1) \epsilon\left[-\partial_{t}^{2} \delta r-\frac{\partial_{t}^{3} \delta r}{n}+\frac{\partial_{t} \partial^{2} \delta r}{n}+\frac{\partial_{t}^{5} \delta r}{n^{3}}-2 \frac{\partial_{t}^{3} \partial^{2} \delta r}{n^{3}}+\frac{\partial_{t} \partial^{2} \partial^{2} \delta r}{n^{3}}+2 \frac{\partial_{t}^{3} \delta r}{n^{2}}\right. \\
\left.-2 \frac{\partial_{t} \partial^{2} \delta r}{n^{2}}+2 \frac{\partial_{t}^{2} \delta r}{n}\right]+\left[-\partial^{2} \delta r-\partial_{t} \partial^{2} \delta r-\frac{\partial^{2} \partial_{t}^{2} \delta r}{n}+\frac{\partial^{2} \partial^{2} \delta r}{n}+2 \frac{\partial^{2} \partial_{t} \delta r}{n}\right. \\
+\frac{\partial^{2} \partial_{t}^{4} \delta r-2 \partial_{t}^{2} \partial^{2} \partial^{2} \delta r+\partial^{2} \partial^{2} \partial^{2} \delta r}{n^{3}}+3 \frac{\partial^{2} \partial_{t}^{2} \delta r-\partial^{2} \partial^{2} \delta r}{n^{2}}+\frac{\partial^{2} \partial_{t}^{2} \delta r}{n^{2}}-\frac{\partial^{2} \partial^{2} \delta r}{n^{2}} \\
\left.+2 \frac{\partial^{2} \partial_{t} \delta r}{n}+2 \frac{\partial^{2} \delta r}{n}\right]=0
\end{array}
$$

We consider the plane wave expansion of the shape fluctuations

$$
\delta r=\delta r^{0} e^{-i \omega t} e^{i k_{a} x^{a}}
$$

We then substitute (4.35) into (4.34) and solve for scalar QNM frequencies (where we take $k \sim \mathcal{O}(\sqrt{n}))^{8}$

$$
\omega_{s}= \pm \frac{k}{\sqrt{n}}\left(1+\frac{1+2 k^{2} / n}{2 n}\right)-\frac{i k^{2}}{n}\left(1-\frac{1}{n}\right), \quad \text { where } k^{2}=k_{a} k^{a} \text { and } k=\sqrt{k^{2}}
$$

Hence we can write the most general solution of (4.34)

$$
\delta r=\delta r_{1}^{0} e^{-i \omega_{1} t} e^{i k_{a} x^{a}}+\delta r_{2}^{0} e^{-i \omega_{2} t} e^{i k_{a} x^{a}}
$$

where,

$$
\begin{aligned}
& \omega_{1}=\frac{k}{\sqrt{n}}\left(1+\frac{1+2 k^{2} / n}{2 n}\right)-\frac{i k^{2}}{n}\left(1-\frac{1}{n}\right), \\
& \omega_{2}=-\frac{k}{\sqrt{n}}\left(1+\frac{1+2 k^{2} / n}{2 n}\right)-\frac{i k^{2}}{n}\left(1-\frac{1}{n}\right)
\end{aligned}
$$

Similarly, we can write the form of the most general solution of (4.33) and (4.32) (Note that there is only one vector QNM frequency)

$$
\delta u_{a}=\delta r_{1}^{0} V_{a}^{1} e^{-i \omega_{1} t} e^{i k_{a} x^{a}}+\delta r_{2}^{0} V_{a}^{2} e^{-i \omega_{2} t} e^{i k_{a} x^{a}}+v_{a} e^{-i \omega_{v} t} e^{i k_{a} x^{a}}
$$

where $V_{a}^{1}$ and $V_{a}^{2}$ are vectors along $k_{a}$, and $v_{a}$ is any vector which satisfies $v_{a} k^{a}=0$.

\footnotetext{
${ }^{8}$ It turns out, as in 1st order, that the orders of temporal and spatial frequencies are related by factor of $\left(\frac{1}{\sqrt{n}}\right)$. This can be seen from the equation (4.33), where there is a relative factor of $(n-1)$ between the divergence of velocity fluctuations and the shape fluctuations. So we cannot have both the temporal and spacial frequencies of the same order.

Here we demanded that the temporal frequency is of order $\mathcal{O}(1)$, but no restriction was put on the spatial frequencies. Such scaling is consistent with the present $\left(\frac{1}{D}\right)$ expansion. See [1] and [5] for detailed explanation.
} 
Substituting (4.39) into (4.33) and (4.32) and solving we find

$$
\begin{aligned}
& \omega_{v}=-\frac{i k^{2}}{n}\left(1+\mathcal{O}\left(n^{-2}\right)\right), \quad V_{a}^{1}=\left[-i\left(1-\frac{1}{n}\right)+\frac{\sqrt{n}}{k}\left(1+\frac{1+2 k^{2} / n}{2 n}\right)\right] k_{a}, \\
& V_{a}^{2}=\left[-i\left(1-\frac{1}{n}\right)-\frac{\sqrt{n}}{k}\left(1+\frac{1+2 k^{2} / n}{2 n}\right)\right] k_{a}
\end{aligned}
$$

Thus, we see that there is no subleading correction to $\omega_{v}$. Collecting the results for light QNM frequencies

$$
\begin{aligned}
& \omega_{s}= \pm \frac{k}{\sqrt{n}}\left(1+\frac{1+2 k^{2} / n}{2 n}\right)-\frac{i k^{2}}{n}\left(1-\frac{1}{n}\right) \\
& \omega_{v}=-\frac{i k^{2}}{n}\left(1+\mathcal{O}\left(n^{-2}\right)\right)
\end{aligned}
$$

Upto the required order, the answers (4.41) agree with the respective answers given in equations (4.23), (4.24), (4.25) of [16].

\section{$5 \quad$ Future directions}

In this note we have found new dynamical 'black-hole' type solutions of the Einstein equations in presence of cosmological constant in an expansion in the inverse powers of dimension. We have done the calculation upto second subleading order. The space-time, determined here, will necessarily possess an event horizon. The dynamics of the horizon could be mapped to the dynamics of a velocity field on a dynamical membrane, embedded in the asymptotic background. We have determined the equation for this dual dynamics of the membrane and the velocity field also in an expansion in $\left(\frac{1}{D}\right)$.

There are several directions along which we could proceed from here.

As we have mentioned in the introduction, one of our key motivation for this second subleading calculation is to have some insight in entropy production, which is expected to take place only at this order. Calculation of this entropy production along with the effective stress tensor for the membrane (see [6] for the stress tensor at first order) could be one immediate project.

As a check we have matched the spectrum of the Quasi-Normal modes. This gives a check on the equation of motion for the membrane. Another important check would be to match the metric with the large dimension limit of known black hole solutions. Apart from just a check on our results, this exercise could also give hints to some exact but non-trivial solutions of our membrane equations. This might lead to some techniques to solve the membrane equation analytically.

It would also be interesting to see how these solutions compare with another perturbative techniques to solve Einstein equations, namely derivative expansion and the correspondence with fluid dynamics (Along these lines, see [7] for a detailed study of the comparison between the Improved large D membrane formalism and the Fluid Gravity). 


\section{Acknowledgments}

It is a great pleasure to thank Shiraz Minwalla for initiating discussions on this topic and for his numerous suggestions throughout the course of this work. We would also like to thank Bidisha Chakrabarty and Arunabha Saha for collaboration at the initial stage. We would like to thank Suman Kundu, Poulomi Nandi for illuminating discussions. P.B. would like to acknowledge the hospitality of ICTS, HRI and SINP while this work was in progress. Y.D. would like to acknowledge the hospitality of ICTS, IIT-Kanpur and IISER-Pune while this work was in progress. The work of Y.D. was supported by the Infosys Endowment for the study of the Quantum Structure of Spacetime, as well as an Indo Israel (UGC/ISF) grant. We would also like to acknowledge our debt to the people of India for their steady and generous support to research in the basic sciences.

\section{A Calculation of the sources $-S_{A B}$}

In this section we shall give the details of the calculation of $S_{A B}$. As mentioned before, the source will be given by $E_{A B}$ calculated on $\left(G_{A B}^{(0)}\right)=g_{A B}+\psi^{-D} O_{A} O_{B}$.

We shall follow appendix(B) of [1] for computation. The first step would be to decompose the source in the following way.

$$
\begin{aligned}
S_{A B} & \left.\equiv E_{A B}\right|_{G_{A B}^{(0)}} \\
& =\left.R_{A B}\right|_{G_{A B}^{(0)}}-(D-1) \lambda G_{A B}^{(0)} \\
& =-(D-1) \lambda \psi^{-D} O_{A} O_{B}+\underbrace{\nabla_{C}\left[\left.\delta \Gamma_{A B}^{C}\right|_{\text {lin }}\right]}_{\left.\delta R_{A B}\right|_{\text {lin }}}+\underbrace{\nabla_{C}\left[\left.\delta \Gamma_{A B}^{C}\right|_{\text {non-lin }}\right]}_{\delta R_{A B}^{(1)} \mid \text { non-lin }} \underbrace{-\left[\delta \Gamma_{B E}^{C}\right]\left[\delta \Gamma_{A C}^{E}\right]}_{\delta R_{A B}^{(2)} \mid \text { non-lin }}
\end{aligned}
$$

where

$$
\begin{aligned}
\left.\delta \Gamma_{B C}^{A}\right|_{\text {lin. }} & =\frac{1}{2}\left\{\nabla_{B}\left(\psi^{-D} O_{C} O^{A}\right)+\nabla_{C}\left(\psi^{-D} O_{B} O^{A}\right)-\nabla^{A}\left(\psi^{-D} O_{B} O_{C}\right)\right\} \\
\left.\delta \Gamma_{B C}^{A}\right|_{\text {non-lin }} & =\frac{1}{2} \psi^{-D} O^{A}(O \cdot \nabla)\left(\psi^{-D} O_{B} O_{C}\right) \\
\delta \Gamma_{B C}^{A} & =\left.\delta \Gamma_{B C}^{A}\right|_{\text {lin. }}+\left.\delta \Gamma_{B C}^{A}\right|_{\text {non-lin. }}
\end{aligned}
$$

At first we present the calculation of $\left.\delta R_{A B}^{(2)}\right|_{\text {non-linear }}$

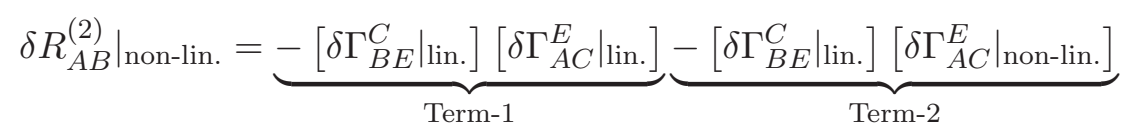

$$
\begin{aligned}
& \underbrace{-\left[\left.\delta \Gamma_{B E}^{C}\right|_{\text {non-lin. }}\right]\left[\left.\delta \Gamma_{A C}^{E}\right|_{\text {lin. }} .\right]}_{\text {Term-3 }} \underbrace{-\left[\left.\delta \Gamma_{B E}^{C}\right|_{\text {non-lin. }}\right]\left[\left.\delta \Gamma_{A C}^{E}\right|_{\text {non-lin. }}\right]}_{\text {Term- } 4}
\end{aligned}
$$

As previously, in this case also, Term- $2=$ Term- $3=$ Term- $4=0$; 
Now we need to calculate Term-1.

$\left.\delta R_{A B}^{(2)}\right|_{\text {non-lin. }}=-\left[\left.\delta \Gamma_{B E}^{C}\right|_{\text {lin. }}\right]\left[\left.\delta \Gamma_{A C}^{E}\right|_{\text {lin. }}\right]$

$$
\begin{aligned}
= & \frac{1}{2} \psi^{-2 D}\left(\nabla_{E} O^{C}\right)\left(\nabla^{E} O_{C}\right) O_{B} O_{A}-\frac{1}{2} \nabla_{E}\left(\psi^{-D} O_{B} O^{C}\right) \nabla_{C}\left(\psi^{-D} O_{A} O^{E}\right) \\
= & -\frac{1}{2}\left[(O \cdot \nabla)\left(\psi^{-D} O_{B}\right)\right]\left[(O \cdot \nabla)\left(\psi^{-D} O_{A}\right)\right]+\psi^{-2 D}\left(\frac{D N}{\psi}\right) Q O_{A} O_{B} \\
& +\frac{\psi^{-2 D}}{2}\left(\nabla_{E} O_{C}\right)\left(\nabla^{E} O^{C}-\nabla^{C} O^{E}\right) O_{B} O_{A}-\psi^{-2 D} Q^{2} O_{B} O_{A}
\end{aligned}
$$

Where, $Q \equiv u^{E}(O \cdot \nabla) n_{E}$

$$
\begin{aligned}
\left.\delta R_{A B}^{(2)}\right|_{\text {non-lin. }}= & -\frac{1}{2}\left[(O \cdot \nabla)\left(\psi^{-D} O_{B}\right)\right]\left[(O \cdot \nabla)\left(\psi^{-D} O_{A}\right)\right]+\psi^{-2 D} K Q O_{A} O_{B} \\
& +\frac{\psi^{-2 D}}{2}\left[\left(\nabla_{E} O_{C}\right)\left(\nabla^{E} O^{C}-\nabla^{C} O^{E}\right)-2 Q^{2}+2 Q \frac{(n \cdot \nabla) K}{K}\right] O_{B} O_{A}
\end{aligned}
$$

In deriving (A.5) we have used,

$$
\frac{D N}{\psi}=K+\frac{(n \cdot \nabla) K}{K}
$$

Now we proceed to the calculation of $\left.\delta R_{A B}^{(1)}\right|_{\text {non-lin. }}$

$\left.\delta R_{A B}^{(1)}\right|_{\text {non-lin. }}$

$$
\begin{aligned}
= & \nabla_{C}\left[\frac{1}{2} \psi^{-D} O^{C}(O \cdot \nabla)\left(\psi^{-D} O_{A} O_{B}\right)\right] \\
= & \left(\frac{\psi^{-D}}{2}\right)\left[(\nabla \cdot O)(O \cdot \nabla)\left(\psi^{-D} O_{B} O_{A}\right)+O_{A}(O \cdot \nabla)\left[(O \cdot \nabla)\left(\psi^{-D} O_{B}\right)\right]\right] \\
& +\frac{1}{2}\left[(O \cdot \nabla)\left(\psi^{-D} O_{A}\right)\right]\left[(O \cdot \nabla)\left(\psi^{-D} O_{B}\right)\right]+\frac{1}{2}(O \cdot \nabla)\left[\psi^{-2 D} O_{B}(O \cdot \nabla) O_{A}\right] \\
= & \frac{1}{2}\left[(O \cdot \nabla)\left(\psi^{-D} O_{A}\right)\right]\left[(O \cdot \nabla)\left(\psi^{-D} O_{B}\right)\right]-\frac{\psi^{-2 D}}{2}(O \cdot \nabla)\left[K O_{A} O_{B}\right] \\
& +\frac{\psi^{-2 D}}{2}\left(\frac{D N}{\psi}-\nabla \cdot O\right)\left(\frac{D N}{\psi}-2 Q\right) O_{A} O_{B} \\
& +\frac{\psi^{-2 D}}{2}\left[3 Q^{2}+2(O \cdot \nabla) Q-(O \cdot \nabla)\left(\frac{(n \cdot \nabla) K}{K}\right)-\frac{(n \cdot \nabla) K}{K} 2 Q\right] O_{A} O_{B}
\end{aligned}
$$

Now,

$$
\begin{aligned}
&\left(\frac{D N}{\psi}-\nabla \cdot O\right)\left(\frac{D N}{\psi}-2 Q\right) \\
&=\left[\frac{(n \cdot \nabla) K}{K}+\frac{(n \cdot \nabla)^{2} K}{K^{2}}-2 \frac{[(n \cdot \nabla) K]^{2}}{K^{3}}+\tilde{\nabla} \cdot u-\frac{1}{K}(u \cdot \nabla)\left(\frac{(n \cdot \nabla) K}{K}\right)\right. \\
&\left.-\frac{(u \cdot \nabla) K}{K}+\frac{1}{K} \frac{(n \cdot \nabla) K}{K} \frac{(u \cdot \nabla) K}{K}\right]\left[K+\frac{(n \cdot \nabla) K}{K}-2 Q\right] \\
&= K(\tilde{\nabla} \cdot u)+(O \cdot \nabla) K+\frac{(n \cdot \nabla)^{2} K}{K}-2\left[\frac{(n \cdot \nabla) K}{K}\right]^{2}+\frac{(O \cdot \nabla) K}{K} \frac{(n \cdot \nabla) K}{K} \\
&-2 Q \frac{(O \cdot \nabla) K}{K}-(u \cdot \nabla)\left(\frac{(n \cdot \nabla) K}{K}\right)+\frac{(n \cdot \nabla) K}{K} \frac{(u \cdot \nabla) K}{K}+(\tilde{\nabla} \cdot u) \frac{(n \cdot \nabla) K}{K}-2 Q(\tilde{\nabla} \cdot u)
\end{aligned}
$$


Where, $\tilde{\nabla}$ is defined in $(2.14)$.

In deriving (A.8) we have used (see B.3 for derivation),

$$
\nabla \cdot u=\tilde{\nabla} \cdot u-\frac{(u \cdot \nabla) K}{K}-\frac{1}{K}(u \cdot \nabla)\left(\frac{(n \cdot \nabla) K}{K}\right)+\frac{1}{K} \frac{(n \cdot \nabla) K}{K} \frac{(u \cdot \nabla) K}{K}
$$

Using, (A.8) we get the final expression of $\left.\delta R_{A B}^{(1)}\right|_{\text {non-lin., }}$

$$
\begin{aligned}
& \left.\delta R_{A B}^{(1)}\right|_{\text {non-lin. }} \\
& =\frac{\psi^{-2 D}}{2} K(\tilde{\nabla} \cdot u) O_{A} O_{B}-\psi^{-2 D} K Q O_{A} O_{B}+\frac{1}{2}\left[(O \cdot \nabla)\left(\psi^{-D} O_{A}\right)\right]\left[(O \cdot \nabla)\left(\psi^{-D} O_{B}\right)\right] \\
& +\frac{\psi^{-2 D}}{2}\left[3 Q^{2}+2(O \cdot \nabla) Q-2 Q\left(\frac{(n \cdot \nabla) K}{K}+\frac{(O \cdot \nabla) K}{K}\right)+(\tilde{\nabla} \cdot u)\left(\frac{(n \cdot \nabla) K}{K}-2 Q\right)\right] O_{A} O_{B}
\end{aligned}
$$

Adding (A.5) and (A.10) we get

$$
\begin{aligned}
& \left.\delta R_{A B}\right|_{\text {non-lin. }} \\
& \left.\equiv \delta R_{A B}^{(1)}\right|_{\text {non-lin. }}+\left.\delta R_{A B}^{(2)}\right|_{\text {non-lin. }} \\
& =\frac{1}{2} \psi^{-2 D} K(\tilde{\nabla} \cdot u) O_{A} O_{B}+\frac{1}{2} \psi^{-2 D}\left[\left(\nabla_{E} O^{C}\right)\left(\nabla^{E} O_{C}-\nabla_{C} O^{E}\right)+Q^{2}+2(O \cdot \nabla) Q\right. \\
& \left.\quad-2 Q \frac{(O \cdot \nabla) K}{K}+(\tilde{\nabla} \cdot u)\left(\frac{(n \cdot \nabla) K}{K}-2 Q\right)\right] O_{A} O_{B}
\end{aligned}
$$

Let us note the presence of ' $K(\tilde{\nabla} \cdot u)$ ' term in $\left.\delta R_{A B}\right|_{\text {non-lin. }}$ From the membrane equation at first subleading order, it follows that this term is of order $\mathcal{O}(1)$ on $\psi=1$ hypersurface. This is sort of 'anomalous', since naive order counting suggests that this term should be or order $\mathcal{O}\left(D^{2}\right)$ and this may not be the case once we are away from the membrane.

Now for any generic term, which is of order $\mathcal{O}(1)$ when evaluated on $(\psi=1)$ hypersurface, will have corrections of order $\mathcal{O}\left(\frac{1}{D}\right)$ (or further suppressed) as one goes away from $\psi=1$. While integrating the ODEs, this is the reason we could ignore all the implicit $\psi$ dependence in the source. However from the above discussion we could see that such reasoning does not work for ' $K(\tilde{\nabla} \cdot u)$ ' (or in fact any such 'anomalous' term). Below we shall examine this term in more detail.

We can expand $(\tilde{\nabla} \cdot u)$ in $\left[\psi-1=\frac{R}{D}\right]$ as follows

$$
\begin{aligned}
\tilde{\nabla} \cdot u= & \left.(\tilde{\nabla} \cdot u)\right|_{\psi=1}+\left.\frac{\psi-1}{N}(n \cdot \nabla)(\tilde{\nabla} \cdot u)\right|_{\psi=1}+\left.\left.\frac{(\psi-1)^{2}}{2 N^{2}}\left[\frac{(n \cdot \nabla) N}{N}\right]\right|_{\psi=1}[(n \cdot \nabla)(\tilde{\nabla} \cdot u)]\right|_{\psi=1} \\
& +\left.\frac{(\psi-1)^{2}}{2 N^{2}}[(n \cdot \nabla)(n \cdot \nabla)(\tilde{\nabla} \cdot u)]\right|_{\psi=1}+\mathcal{O}(\psi-1)^{3} \\
= & \left.(\tilde{\nabla} \cdot u)\right|_{R=0}-R\left[\frac{\tilde{\nabla} \cdot E}{K}\right]_{R=0}-\frac{R^{2}}{2}\left[\left(\frac{(n \cdot \nabla) K}{K^{3}}\right)(\tilde{\nabla} \cdot E)\right]_{R=0} \\
& +R^{2}\left[\left(\frac{D^{2}}{K^{3}}\right) \mathfrak{s}_{2}\right]_{R=0}+\mathcal{O}\left(\frac{1}{D}\right)^{2} \\
= & \left.(\tilde{\nabla} \cdot u)\right|_{R=0}-R\left[\frac{\tilde{\nabla} \cdot E}{K}\right]_{R=0}+R^{2}\left[\left(\frac{D^{2}}{K^{3}}\right) \mathfrak{s}_{2}\right]_{R=0}+\mathcal{O}\left(\frac{1}{D}\right)^{2}
\end{aligned}
$$


Where $E_{A}$ is given in equation (3.8).

In the second line we have used the following two identities (to prove them we have used Mathematica Version-11),

$$
\begin{array}{r}
\left.(n \cdot \nabla)(\tilde{\nabla} \cdot u)\right|_{R=0}=-\left.(\tilde{\nabla} \cdot E)\right|_{R=0}+\mathcal{O}\left(\frac{1}{D}\right) \\
\left.(n \cdot \nabla)(n \cdot \nabla)(\tilde{\nabla} \cdot u)\right|_{R=0}=\left.2 D^{2}\left(\frac{\mathfrak{s}_{2}}{K}\right)\right|_{R=0}+\mathcal{O}(1)
\end{array}
$$

Clearly the second and the third term in the last line of equation (A.12) (which encode the value of $(\tilde{\nabla} \cdot u)$ off the membrane) could contribute in $\left.\delta R_{A B}\right|_{\text {non-lin. }}$ at order $\mathcal{O}(1)$.

Substituting (A.12) in equation (A.11) we find

$\left.\delta R_{A B}\right|_{\text {non-lin. }}$

$$
\begin{aligned}
& =\psi^{-2 D}\left(\frac{K}{2}\right)\left[(\tilde{\nabla} \cdot u)_{\psi=1}-R\left(\frac{\tilde{\nabla} \cdot E}{K}\right)_{\psi=1}-\frac{1}{2 K}\left[\nabla_{(E} u_{F)} \nabla_{(C} u_{D)} P^{F C} P^{E D}\right]\right] O_{A} O_{B} \\
& +\frac{\psi^{-2 D}}{2} R^{2}\left(\frac{D^{2}}{K^{2}}\right)\left(\mathfrak{s}_{2}\right) O_{A} O_{B} \\
& -\psi^{-2 D}\left[2 u^{A} K_{A}^{C} \frac{\nabla_{C} K}{K}-\left(\nabla_{C} u_{A}\right)\left(\nabla^{C} u^{A}\right)-(u \cdot K \cdot K \cdot u)+3\left(\frac{(u \cdot \nabla) K}{K}\right)^{2}\right. \\
& \left.-\frac{K}{D}\left(\frac{(u \cdot \nabla) K}{K}\right)+\frac{K}{D}(u \cdot K \cdot u)-2 \frac{(u \cdot \nabla) K}{K}(u \cdot K \cdot u)-u^{E} u^{F} \bar{R}_{E D F C} O^{C} O^{D}\right] O_{A} O_{B} \\
& =\psi^{-2 D}\left(\frac{K}{2}\right)\left[(\tilde{\nabla} \cdot u)_{\psi=1}-R\left(\frac{\tilde{\nabla} \cdot E}{K}\right)_{\psi=1}-\frac{1}{2 K}\left[\nabla_{(E} u_{F)} \nabla_{(C} u_{D)} P^{F C} P^{E D}\right]\right] O_{A} O_{B} \\
& +\frac{\psi^{-2 D}}{2} R^{2}\left(\frac{D^{2}}{K^{2}}\right)\left(\mathfrak{s}_{2}\right) O_{A} O_{B}-\psi^{-2 D}\left[\left(\frac{u \cdot \nabla K}{K}\right)^{2}+4 u^{A} K_{A}^{B} \frac{\nabla_{B} K}{K}-\left(\tilde{\nabla}_{A} u_{B}\right)\left(\tilde{\nabla}^{A} u^{B}\right)\right. \\
& -(u \cdot K \cdot u)^{2}-2 \frac{\tilde{\nabla}^{A} K}{K}\left[(u \cdot \nabla) u_{A}\right]-\left[(u \cdot \tilde{\nabla}) u_{A}\right]\left[(u \cdot \tilde{\nabla}) u^{A}\right]+2\left[(u \cdot \nabla) u^{A}\right]\left(u^{B} K_{B A}\right) \\
& \left.-3(u \cdot K \cdot K \cdot u)-\frac{\tilde{\nabla}_{A} K}{K} \frac{\tilde{\nabla}^{A} K}{K}-\frac{K}{D}\left(\frac{u \cdot \nabla K}{K}-u \cdot K \cdot u\right)+u^{E} u^{F} n^{D} n^{C} \bar{R}_{C E F D}\right] O_{A} O_{B} \\
& =e^{-2 R}\left(\frac{K}{2}\right)\left[(\tilde{\nabla} \cdot u)_{R=0}-\frac{1}{2 K}\left(\nabla_{E} u_{F}+\nabla_{F} u_{E}\right)\left(\nabla_{C} u_{D}+\nabla_{D} u_{C}\right) P^{F C} P^{E D}\right] O_{A} O_{B} \\
& +\left(\frac{e^{-2 R}}{2}\right)\left[-R(\tilde{\nabla} \cdot E)_{R=0}+R^{2}\left(\frac{D^{2}}{K^{2}} \mathfrak{s}_{2}\right)_{R=0}\right] O_{A} O_{B}-e^{-2 R}\left(\mathfrak{s}_{1}\right) O_{A} O_{B}
\end{aligned}
$$

where

$$
\begin{aligned}
\mathfrak{s}_{1}= & u^{E} u^{F} n^{D} n^{C} \bar{R}_{C E F D}+\left(\frac{u \cdot \nabla K}{K}\right)^{2}+\frac{\tilde{\nabla}_{A} K}{K}\left[4 u^{B} K_{B}^{A}-2\left[(u \cdot \nabla) u_{A}\right]-\frac{\tilde{\nabla}^{A} K}{K}\right] \\
& -\left(\tilde{\nabla}_{A} u_{B}\right)\left(\tilde{\nabla}^{A} u^{B}\right)-(u \cdot K \cdot u)^{2}-\left[(u \cdot \tilde{\nabla}) u_{A}\right]\left[(u \cdot \tilde{\nabla}) u^{A}\right]+2\left[(u \cdot \nabla) u^{A}\right]\left(u^{B} K_{B A}\right) \\
& -3(u \cdot K \cdot K \cdot u)-\frac{K}{D}\left(\frac{u \cdot \nabla K}{K}-u \cdot K \cdot u\right)
\end{aligned}
$$




$$
\begin{aligned}
\mathfrak{s}_{2}=\frac{K^{2}}{D^{2}}[ & -\frac{K}{D}\left(\frac{u \cdot \nabla K}{K}-u \cdot K \cdot u\right)-2 \lambda-(u \cdot K \cdot K \cdot u)+2\left(\frac{\nabla_{A} K}{K}\right) u^{B} K_{B}^{A}-\left(\frac{u \cdot \nabla K}{K}\right)^{2} \\
& \left.+2\left(\frac{u \cdot \nabla K}{K}\right)(u \cdot K \cdot u)-\left(\frac{\tilde{\nabla}^{D} K}{K}\right)\left(\frac{\tilde{\nabla}_{D} K}{K}\right)-(u \cdot K \cdot u)^{2}+n^{B} n^{D} u^{E} u^{F} \bar{R}_{F B D E}\right]
\end{aligned}
$$

Now we shall calculate those terms in Ricci tensor that are linear in $\psi^{-D}$

$$
\begin{aligned}
\left.\delta R_{A B}\right|_{\text {lin. }}= & \nabla_{C}\left[\delta \Gamma_{B A}^{C}||_{\text {lin. }}\right] \\
= & \underbrace{\frac{1}{2} \nabla_{C}\left\{\nabla_{B}\left(\psi^{-D} O_{A} O^{C}\right)\right\}}_{T_{1}}+\underbrace{\frac{1}{2} \nabla_{C}\left\{\nabla_{A}\left(\psi^{-D} O_{B} O^{C}\right)\right\}}_{T_{3}} \underbrace{\frac{1}{2} \nabla_{C}\left\{\nabla_{B}\left(\psi^{-D} O_{A} O^{C}\right)\right\}}_{T_{1}=} \\
= & \frac{1}{2}\left[\nabla_{C}, \nabla_{B}\right]\left(\psi^{-D} O_{A} O^{C}\right)+\frac{1}{2} \nabla_{B} \nabla_{C}\left(\psi^{-D} \mathcal{\psi}_{A} O^{C}\right) \\
= & \left.\frac{\psi^{-D}}{2}\left(\bar{R}_{B D} O^{D} O_{B}\right)\right\} \\
= & \frac{\psi^{-D}}{2}\left(\bar{R}_{B D} O^{D} O_{A}+\bar{R}_{C B A D} O^{D} O^{C}\right)-\frac{1}{2} \nabla_{B}\left[\psi^{-D}\left\{\left(\frac{D N}{\psi}-\nabla \cdot O\right) O_{A}-Q O_{A} O^{C}\right)+\left(\frac{D N}{2 \psi}\right) \psi^{-D}\left[\frac{D N}{\psi}-\nabla \cdot O-Q\right] n_{B} O_{A}\right. \\
& -\frac{1}{2} \psi^{-D} \nabla_{B}\left\{\left(\frac{D N}{\psi}-\nabla \cdot O-Q\right) O_{A}\right\} \\
= & \frac{\psi^{-D}}{2}\left(\bar{R}_{B D} O^{D} O_{A}+\bar{R}_{C B A D} O^{D} O^{C}\right)+\frac{\psi^{-D}}{2}[(n \cdot \nabla) K+K(\nabla \cdot u-Q)] n_{B} O_{A} \\
+ & \frac{\psi^{-D}}{2}\left[\frac{(n \cdot \nabla)^{2} K}{K}-2\left(\frac{(n \cdot \nabla) K}{K}\right)^{2}-\frac{K}{D}\left(\frac{(n \cdot \nabla) K}{K}\right)\right] n_{B} O_{A}+\frac{\psi^{-D}}{2}\left(\frac{K}{D}\right)\left(\nabla_{B} O_{A}\right) \\
& -\frac{\psi^{-D}}{2} O_{A} \nabla_{B}\left[\frac{(n \cdot \nabla) K}{K}-2 \frac{(u \cdot \nabla) K}{K}+u \cdot K \cdot u+\tilde{\nabla} \cdot u\right]
\end{aligned}
$$

Similarly, we will get $T_{2}$ by interchanging $A$ and $B$ indices

$$
\begin{aligned}
T_{3} & =-\frac{1}{2} \nabla_{C} \nabla^{C}\left(\psi^{-D} O_{B} O_{A}\right) \\
& =-\frac{1}{2}\left(\nabla^{2} \psi^{D}\right) O_{A} O_{B}-\left(\nabla_{C} \psi^{-D}\right)\left(\nabla^{C} O_{A} O_{B}\right)-\frac{\psi^{-D}}{2} \nabla^{2}\left(O_{A} O_{B}\right) \\
& =\psi^{-D}\left[\left(\frac{D N}{\psi}\right)(n \cdot \nabla)\left(O_{A} O_{B}\right)-\frac{1}{2} \nabla^{2}\left(O_{A} O_{B}\right)\right]
\end{aligned}
$$

Adding $T_{1}, T_{2}, T_{3}$ we get the expression for $\left.\delta R_{A B}\right|_{\operatorname{lin}}$

$$
\begin{aligned}
\delta R_{A B} & \left.\right|_{\operatorname{lin}} \\
= & \psi^{-D}(D-1) \lambda O_{A} O_{B}+\psi^{-D} \bar{R}_{C A B D} O^{D} O^{C}+\psi^{-D} K(n \cdot \nabla)\left(O_{A} O_{B}\right) \\
& +\frac{\psi^{-D}}{2}\left(n_{B} O_{A}+n_{A} O_{B}\right)[(n \cdot \nabla) K+K(\nabla \cdot u-Q)]-\frac{\psi^{-D}}{2}\left(O_{A} \nabla^{2} O_{B}+O_{B} \nabla^{2} O_{A}\right) \\
& +\frac{\psi^{-D}}{2}\left\{\frac{(n \cdot \nabla)^{2} K}{K}-2\left[\frac{(n \cdot \nabla) K}{K}\right]^{2}-\frac{K}{D} \frac{(n \cdot \nabla) K}{K}\right\}\left(n_{B} O_{A}+O_{B} n_{A}\right)
\end{aligned}
$$




$$
\begin{aligned}
& +\psi^{-D}\left\{\left[\frac{(n \cdot \nabla) K}{K}\right](n \cdot \nabla)\left(O_{A} O_{B}\right)-\left(\nabla_{C} O_{A}\right)\left(\nabla^{C} O_{B}\right)\right\}+\frac{\psi^{-D}}{2} \frac{K}{D}\left[\nabla_{B} O_{A}+\nabla_{A} O_{B}\right] \\
& -\frac{\psi^{-D}}{2}\left(O_{A} \nabla_{B}+O_{B} \nabla_{A}\right)\left[\frac{(n \cdot \nabla) K}{K}-2 \frac{(u \cdot \nabla) K}{K}+u \cdot K \cdot u+\tilde{\nabla} \cdot u\right]
\end{aligned}
$$

Now, we shall decompose the source in the way as mentioned in (3.5). Note that the decomposition of a general 2-index symmetric tensor $\left(C_{A B}\right)$ is the following

$$
\begin{aligned}
C_{A B}= & P_{A}^{D} P_{B}^{E} C_{D E}+\left(P_{A}^{E} O_{B}+P_{B}^{E} O_{A}\right) C_{E D} u^{D}+\left(P_{A}^{E} n_{B}+P_{B}^{E} n_{A}\right) C_{E D} O^{D} \\
& +\left(n_{A} O_{B}+n_{B} O_{A}\right)\left(O^{E} C_{E D} u^{D}\right)+O_{A} O_{B}\left(u^{E} C_{E D} u^{D}\right)+n_{A} n_{B}\left(O^{E} C_{E D} O^{D}\right)
\end{aligned}
$$

Using (A.20) we shall first decompose each of the tensor structure appearing in (A.19)

$$
\begin{array}{rl}
(n \cdot \nabla)\left(O_{A} O_{B}\right)= & 2\left[u^{C}(n \cdot \nabla) n_{C}\right] O_{A} O_{B}+\left(O_{A} P_{B}^{C}+O_{B} P_{A}^{C}\right)(n \cdot \nabla) O_{C} \\
= & 2\left[u^{C}(n \cdot \nabla) n_{C}\right] O_{A} O_{B}+\left(O_{A} P_{B}^{C}+O_{B} P_{A}^{C}\right)(u \cdot \nabla) O_{C} \\
O_{B} \nabla^{2} O_{A}+O_{A} \nabla^{2} O_{B} & (\text { A.21 }) \\
=2\left[K\left[u^{D}(n \cdot \nabla) n_{D}\right]+(u \cdot \nabla) K-u^{D} K_{D}^{C}\left(\frac{\nabla_{C} K}{K}\right)+u^{D}(n \cdot \nabla)^{2} n_{D}+\left(\nabla_{C} u_{D}\right)\left(\nabla^{C} u^{D}\right)\right] O_{A} O_{B} \\
-\left[\left(\nabla^{C} O_{D}\right)\left(\nabla_{C} O^{D}\right)\right]\left[n_{A} O_{B}+n_{B} O_{A}\right]+\left(O_{B} P_{A}^{C}+O_{A} P_{B}^{C}\right) \nabla^{2} O_{C} \\
\left(\nabla_{C} O_{A}\right)\left(\nabla^{C} O_{B}\right)= & \left(u^{D} \nabla_{C} n_{D}\right)\left(u^{E} \nabla^{C} n_{E}\right) O_{A} O_{B}+\left(\nabla_{D} O_{C}\right)\left(\nabla^{D} O_{C^{\prime}}\right) P_{A}^{C} P_{B}^{C^{\prime}} \\
& +\left(O_{B} P_{A}^{C}+O_{A} P_{B}^{C}\right)\left[\left(\nabla_{F} O_{C}\right)\left(u^{D} \nabla^{F} n_{D}\right)\right] \\
\nabla_{B} O_{A}+\nabla_{A} O_{B}=2 & 2(u \cdot K \cdot u) O_{A} O_{B}+Q\left(n_{A} O_{B}+n_{B} O_{A}\right)+P_{A}^{C} P_{B}^{C^{\prime}}\left(\nabla_{C} O_{C^{\prime}}+\nabla_{C^{\prime}} O_{C}\right) \\
& +\left(O_{B} P_{A}^{C}+O_{A} P_{B}^{C}\right)\left[(u \cdot \nabla) O_{C}+u^{D} K_{C D}\right] \\
\left(O_{A} \nabla_{B}+O_{B} \nabla_{A}\right) & {\left[\frac{(n \cdot \nabla) K}{K}-2 \frac{(u \cdot \nabla) K}{K}+u \cdot K \cdot u+\tilde{\nabla}^{2} \cdot u\right]} \\
=-2 & \frac{(u \cdot \nabla) K}{D} O_{A} O_{B}-\left(O_{A} P_{B}^{C}+O_{B} P_{A}^{C}\right) \frac{\nabla_{C} K}{D} \\
+ & \left(O_{A} n_{B}+O_{B} n_{A}\right)(O \cdot \nabla)\left[\frac{(n \cdot \nabla) K}{K}-2 \frac{(u \cdot \nabla) K}{K}+u \cdot K \cdot u+\tilde{\nabla} \cdot u\right] \\
\bar{R}_{C A B D} O^{D} O^{C}= & P_{A}^{E} P_{B}^{F} \bar{R}_{C E F D} O^{D} O^{C}+O_{A} O_{B} u^{E} u^{F} \bar{R}_{C E F D} O^{D} O^{C} \\
& +\left(P_{A}^{E} O_{B}+P_{B}^{E} O_{A}\right) \bar{R}_{C E F D} O^{D} O^{C} u^{F}
\end{array}
$$

Using (A.21), (A.22), (A.23), (A.24), (A.25) we can decompose $\left.\delta R_{A B}\right|_{\text {lin }}$ in the following way

$$
\begin{aligned}
\left.\delta R_{A B}\right|_{\operatorname{lin}}= & \delta R_{\operatorname{lin}}^{\left(S_{1}\right)} O_{A} O_{B}+\delta R_{\operatorname{lin}}^{\left(S_{2}\right)}\left(n_{A} O_{B}+n_{B} O_{A}\right)+\delta R_{\operatorname{lin}}^{\left(S_{3}\right)} n_{A} n_{B}+\delta R_{\operatorname{lin}}^{(t r)} P_{A B} \quad(\mathrm{~A} .27) \\
& +\left(O_{A} P_{B}^{C}+O_{B} P_{A}^{C}\right)\left[\delta R_{\operatorname{lin}}^{\left(V_{1}\right)}\right]_{C}+\left(n_{A} P_{B}^{C}+n_{B} P_{A}^{C}\right)\left[\delta R_{\operatorname{lin}}^{\left(V_{2}\right)}\right]_{C}+\left[\delta R_{\operatorname{lin}}^{(T)}\right]_{A B}
\end{aligned}
$$


Where

$$
\begin{aligned}
\delta R^{\left(S_{1}\right)}=\psi^{-D}(D-1) \lambda+\psi^{-D}\left[u^{E} u^{F} \bar{R}_{C E F D} n^{D} n^{C}-(u \cdot \nabla)\left(\frac{(n \cdot \nabla) K}{K}\right)+u^{A} K_{A}^{C} \frac{\nabla_{C} K}{K}\right. \\
-\frac{(n \cdot \nabla) K}{K} \frac{(u \cdot \nabla) K}{K}+2 \frac{(n \cdot \nabla) K}{K}\left[u^{C}(n \cdot \nabla) n_{C}\right]-\left(u^{D} \nabla_{C} n_{D}\right)\left(u^{E} \nabla^{C} n_{E}\right) \\
\left.-u^{A}(n \cdot \nabla)^{2} n_{A}-\left(\nabla_{C} u_{A}\right)\left(\nabla^{C} u^{A}\right)+\frac{K}{D}(u \cdot K \cdot u)+\frac{K}{D} \frac{(u \cdot \nabla) K}{K}\right] \\
=\psi^{-D}(D-1) \lambda+\psi^{-D}\left[2 u^{A} K_{A}^{C} \frac{\nabla_{C} K}{K}-\left(\nabla_{C} u_{A}\right)\left(\nabla^{C} u^{A}\right)-(u \cdot K \cdot K \cdot u)-\frac{K}{D} \frac{(u \cdot \nabla) K}{K}\right. \\
\left.+3\left(\frac{(u \cdot \nabla) K}{K}\right)^{2}+\frac{K}{D}(u \cdot K \cdot u)-2 \frac{(u \cdot \nabla) K}{K}(u \cdot K \cdot u)+u^{E} u^{F} \bar{R}_{C E F D} n^{D} n^{C}\right] \\
=\psi^{-D}(D-1) \lambda+\psi^{-D} \mathfrak{s}_{1}
\end{aligned}
$$

Where,

$$
\begin{aligned}
\mathfrak{s}_{1}= & \left(\frac{u \cdot \nabla K}{K}\right)^{2}+\frac{\tilde{\nabla}_{A} K}{K}\left[4 u^{B} K_{B}^{A}-2\left[(u \cdot \nabla) u_{A}\right]-\frac{\tilde{\nabla}^{A} K}{K}\right]-\left(\tilde{\nabla}_{A} u_{B}\right)\left(\tilde{\nabla}^{A} u^{B}\right) \\
& -(u \cdot K \cdot u)^{2}-\left[(u \cdot \tilde{\nabla}) u_{A}\right]\left[(u \cdot \tilde{\nabla}) u^{A}\right]+2\left[(u \cdot \nabla) u^{A}\right]\left(u^{B} K_{B A}\right)-3(u \cdot K \cdot K \cdot u) \\
& -\frac{K}{D}\left(\frac{u \cdot \nabla K}{K}-u \cdot K \cdot u\right)+u^{E} u^{F} \bar{R}_{C E F D} n^{D} n^{C} \\
\delta R^{\left(S_{2}\right)}= & \frac{\psi^{-D}}{2}\left[K\left\{\tilde{\nabla} \cdot u-\frac{(u \cdot \nabla) K}{K}-\frac{1}{K}(u \cdot \nabla)\left(\frac{(n \cdot \nabla) K}{K}\right)+\frac{1}{K} \frac{(n \cdot \nabla) K}{K} \frac{(u \cdot \nabla) K}{K}\right\}\right. \\
& +(n \cdot \nabla) K-K Q+\frac{(n \cdot \nabla)^{2} K}{K}-2\left(\frac{(n \cdot \nabla) K}{K}\right)^{2}-\frac{K}{D} \frac{(n \cdot \nabla) K}{K}+\frac{K}{D} Q \\
& \left.+\left(\nabla^{C} O_{A}\right)\left(\nabla_{C} O^{A}\right)-(O \cdot \nabla)\left(\frac{(n \cdot \nabla) K}{K}-2 \frac{(u \cdot \nabla) K}{K}+u \cdot K \cdot u+\tilde{\nabla} \cdot u\right)\right]
\end{aligned}
$$

We shall massage the above expression for $\delta R^{\left(S_{2}\right)}$ a little more.

Let us note the presence of ' $K(\tilde{\nabla} \cdot u)$ ' term in $\delta R^{\left(S_{2}\right)}$. From the discussion just below the equation (A.11) it is clear that we need to take the expansion of $\tilde{\nabla} \cdot u$ in $\psi-1$. The $\psi-1$ expansion of $(\tilde{\nabla} \cdot u)$ is given by $(\mathrm{A} .12)$

$$
\tilde{\nabla} \cdot u=(\tilde{\nabla} \cdot u)_{R=0}-R\left[\frac{\tilde{\nabla} \cdot E}{K}\right]_{R=0}+R^{2}\left[\left(\frac{D^{2}}{K^{3}}\right) \mathfrak{s}_{2}\right]_{R=0}+\mathcal{O}\left(\frac{1}{D}\right)^{2}
$$

Substituting equation (A.31) in equation (A.30) we find

$$
\begin{aligned}
\delta R^{\left(S_{2}\right)}= & \frac{\psi^{-D}}{2}\left[K(\tilde{\nabla} \cdot u)_{R=0}-R(\tilde{\nabla} \cdot E)_{R=0}+R^{2}\left[\left(\frac{D^{2}}{K^{2}}\right) \mathfrak{s}_{2}\right]_{R=0}\right] \\
& +\frac{\psi^{-D}}{2}\left[-K\left\{\frac{(u \cdot \nabla) K}{K}+\frac{1}{K}(u \cdot \nabla)\left(\frac{(n \cdot \nabla) K}{K}\right)-\frac{1}{K} \frac{(n \cdot \nabla) K}{K} \frac{(u \cdot \nabla) K}{K}\right\}\right.
\end{aligned}
$$




$$
\begin{aligned}
& +(n \cdot \nabla) K-K Q+\frac{(n \cdot \nabla)^{2} K}{K}-2\left(\frac{(n \cdot \nabla) K}{K}\right)^{2}-\frac{K}{D} \frac{(n \cdot \nabla) K}{K}+\frac{K}{D} Q \\
& \left.+\left(\nabla^{C} O_{A}\right)\left(\nabla_{C} O^{A}\right)-(O \cdot \nabla)\left(\frac{(n \cdot \nabla) K}{K}-2 \frac{(u \cdot \nabla) K}{K}+u \cdot K \cdot u+\tilde{\nabla} \cdot u\right)\right]
\end{aligned}
$$

Now it turns out that it is possible to rewrite the last three lines of equation (A.32) in terms of the already defined scalar structures $\mathfrak{s}_{1}$ plus few extra terms which could be expressed as functions of membrane equation.

We have used Mathematica Version 11 for this purpose ${ }^{9}$

$$
\begin{aligned}
\delta R^{\left(S_{2}\right)}= & \left.e^{-R}\left[-\mathfrak{s}_{1}+\frac{K}{2}\left((\tilde{\nabla} \cdot u)-\frac{1}{2 K} \nabla_{\left(A u_{B)}\right.} \nabla_{(C} u_{D)} P^{A C} P^{B D}\right)\right]\right|_{R=0} \\
& +\frac{e^{-R}}{2}\left[-R(\tilde{\nabla} \cdot E)_{R=0}+R^{2}\left[\left(\frac{D^{2}}{K^{2}}\right) \mathfrak{s}_{2}\right]_{R=0}\right]+\mathcal{O}\left(\frac{1}{D}\right)^{2}
\end{aligned}
$$

This type of rewriting helps to see the consistency of the set of coupled ODEs manifestly (see section 4.1).

Let us continue with derivation for the rest of the components of the source.

$$
\begin{aligned}
\delta R^{\left(S_{3}\right)} & =0 \\
\delta R^{(t r)} & =\frac{\psi^{-D}}{2} \frac{P^{C C^{\prime}}}{D-2}\left[-2\left(\nabla_{D} O_{C}\right)\left(\nabla^{D} O_{C^{\prime}}\right)+\frac{K}{D}\left(\nabla_{C} O_{C^{\prime}}+\nabla_{C^{\prime}} O_{C}\right)\right] \\
& =\frac{\psi^{-D}}{2} \frac{1}{D-2}\left[-2 P^{C C^{\prime}}\left(\nabla_{D} n_{C}\right)\left(\nabla^{D} n_{C^{\prime}}\right)+\frac{K}{D} P^{C C^{\prime}}\left(\nabla_{C^{\prime}} n_{C^{\prime}}+\nabla_{C^{\prime}} n_{C}\right)\right]+\mathcal{O}\left(\frac{1}{D}\right) \\
& =\frac{\psi^{-D}}{2} \frac{1}{D-2}\left(-2 \frac{K^{2}}{D}+2 \frac{K^{2}}{D}\right)+\mathcal{O}\left(\frac{1}{D}\right) \\
& =0 \\
{\left[\delta R_{\operatorname{lin}}^{\left(V_{1}\right)}\right.} & ]_{A} \\
= & \frac{\psi^{-D}}{2} P_{A}^{C}\left[2 K(u \cdot \nabla) O_{C}-\nabla^{2} O_{C}\right]+\frac{\psi^{-D}}{2} P_{A}^{C}\left[2 \bar{R}_{E C F D} O^{D} O^{E} u^{F}\right.
\end{aligned}
$$

${ }^{9}$ More precisely Mathematica has been used to rearrange $\delta R^{\left(S_{2}\right)}$ on $R=0$ hypersurface . Away from the membrane the calculation is relatively less tedious and could be done by hand. On $\psi=1$ i.e., on $\mathrm{R}=0$, $\delta R^{\left(S_{2}\right)}$ becomes

$$
\begin{aligned}
& \left.\quad \delta R^{\left(S_{2}\right)}\right|_{R=0}=\left.e^{-R}\left[-\mathfrak{s}_{1}+\frac{K}{2}\left((\tilde{\nabla} \cdot u)-\frac{1}{2 K} \nabla_{(A} u_{B)} \nabla_{(C} u_{D)} P^{A C} P^{B D}\right)\right]\right|_{R=0} \\
& \text { Where, } \quad \nabla_{(A} u_{B)}=\nabla_{A} u_{B}+\nabla_{B} u_{A} .
\end{aligned}
$$

For Mathematica computation we do have to choose a specific background and coordinate system. Since we have an independent proof that the final answer is 'background-covariant', such a choice does not imply any loss of generality. However, we need to do an appropriate 'geometrization' of the answer that we get from Mathematica, so that we could write it in a 'background covariant form' as desired. See [3, 4] for details of this procedure. 


$$
\begin{aligned}
& \left.+2 \frac{(n \cdot \nabla) K}{K}\left[(u \cdot \nabla) O_{C}\right]+\frac{\nabla_{C} K}{D} 2\left(\nabla_{F} O_{C}\right)\left(u^{D} \nabla^{F} n_{D}\right)+\frac{K}{D}(u \cdot \nabla) O_{C}+\frac{K}{D}\left(u^{D} K_{C D}\right)\right] \\
= & \left.\frac{e^{-R}}{2} P_{A}^{C}\left[2 K(u \cdot \nabla) O_{C}-\nabla^{2} O_{C}\right]\right|_{\psi=1}+\left.\frac{e^{-R}}{2}\left(\frac{\psi-1}{N}\right)(n \cdot \nabla)\left[P_{A}^{C}\left(2 K(u \cdot \nabla) O_{C}-\nabla^{2} O_{C}\right)\right]\right|_{\psi=1} \\
& +\frac{e^{-R}}{2} P_{A}^{C}\left[2 \bar{R}_{E C F D} O^{D} O^{E} u^{F}+2 \frac{(n \cdot \nabla) K}{K}\left[(u \cdot \nabla) O_{C}\right]+\frac{\nabla_{C} K}{D}\right. \\
& \left.-2\left(\nabla_{F} O_{C}\right)\left(u^{D} \nabla^{F} n_{D}\right)+\frac{K}{D}(u \cdot \nabla) O_{C}+\frac{K}{D} u^{D} K_{C D}\right] \\
= & \left(\frac{e^{-R}}{2}\right)\left[K E_{A}^{\text {vector }}-2 R\left(\frac{D}{K}\right) \mathfrak{v}_{A}\right]
\end{aligned}
$$

In the last line we have used the following two identities (see appendix B.4 and B.5 for derivation)

$$
\begin{aligned}
& (n \cdot \nabla)\left[P_{A}^{C}\left(2 K(u \cdot \nabla) O_{C}-\nabla^{2} O_{C}\right)\right]_{R=0}=-2 D \mathfrak{v}_{A} \\
& P_{A}^{C}\left[2 K(u \cdot \nabla) O_{C}-\nabla^{2} O_{C}+2 \bar{R}_{E C F D} O^{D} O^{E} u^{F}+2 \frac{(n \cdot \nabla) K}{K}\left[(u \cdot \nabla) O_{C}\right]+\frac{\nabla_{C} K}{D}\right. \\
& \left.\quad-2\left(\nabla_{F} O_{C}\right)\left(u^{D} \nabla^{F} n_{D}\right)+\frac{K}{D}(u \cdot \nabla) O_{C}+\frac{K}{D} u^{D} K_{C D}\right]_{\psi=1}=K E_{A}^{\text {vector }}
\end{aligned}
$$

Where $E_{A}^{\text {vector }}$ is the subleading (see equation (3.8)) membrane equation, and $\mathfrak{v}_{A}$ is given by

$$
\begin{aligned}
\mathfrak{v}_{A}=P_{A}^{B}[ & \frac{K}{D}\left(n^{D} u^{E} O^{F} \bar{R}_{F B D E}\right)+\frac{K^{2}}{2 D^{2}}\left(\frac{\nabla_{B} K}{K}+(u \cdot \nabla) u_{B}-2 u^{D} K_{D B}\right) \\
& \left.-P^{F D}\left(\frac{\nabla_{F} K}{D}-\frac{K}{D}\left(u^{E} K_{E F}\right)\right)\left(K_{D B}-\nabla_{D} u_{B}\right)\right]
\end{aligned}
$$

Note that the simplification of $\left[\delta R_{\text {lin }}^{\left(V_{1}\right)}\right]$ involves the same issues as in $\delta R^{\left(S_{2}\right)}$. The first line of the r.h.s. of equation (A.37) is of order $\mathcal{O}(D)$ by naive order counting. However, because of the membrane equation at first subleading order, this is of $\mathcal{O}(1)$ on $\psi=1$ hypersurface. Away from the hypersurface this may not be the case and we have to expand the first line around $\psi=1$ and take into account at least the first term in the expansion. This is what has been done in the second line of equation (A.37). In the final step we have re-written $\left[\delta R_{\text {lin }}^{\left(V_{1}\right)}\right]$ in terms of already-defined vector structure $\mathfrak{v}_{A}$ plus terms proportional to membrane equation.

The rest of the components of $S_{A B}$ are easy to compute without any further subtlety.

$$
\begin{aligned}
& {\left[\delta R_{\operatorname{lin}}^{\left(V_{2}\right)}\right]_{C}=0} \\
& {\left[\delta R_{\operatorname{lin}}^{(T)}\right]_{A B}} \\
& \quad=\frac{\psi^{-D}}{2} P_{A}^{C} P_{B}^{C^{\prime}}\left[2 \bar{R}_{F C C^{\prime} D} O^{D} O^{F}-2\left(\nabla_{D} O_{C}\right)\left(\nabla^{D} O_{C^{\prime}}\right)+\frac{K}{D}\left(\nabla_{C} O_{C^{\prime}}+\nabla_{C^{\prime}} O_{C}\right)\right]
\end{aligned}
$$




$$
\begin{aligned}
& -\frac{\psi^{-D}}{2} \frac{P_{A B}}{D-2} P^{C C^{\prime}}\left[-2\left(\nabla_{D} O_{C}\right)\left(\nabla^{D} O_{C^{\prime}}\right)+\frac{K}{D}\left(\nabla_{C} O_{C^{\prime}}+\nabla_{C^{\prime}} O_{C}\right)\right] \\
= & \frac{\psi^{-D}}{2} P_{A}^{C} P_{B}^{C^{\prime}}\left[2 \bar{R}_{F C C^{\prime} D} O^{D} O^{F}-2\left(\nabla_{D} O_{C}\right)\left(\nabla^{D} O_{C^{\prime}}\right)+\frac{K}{D}\left(\nabla_{C} O_{C^{\prime}}+\nabla_{C^{\prime}} O_{C}\right)\right] \\
= & \psi^{-D} P_{A}^{C} P_{B}^{C^{\prime}}\left[\frac{K}{D}\left(K_{C C^{\prime}}-\frac{\nabla_{C} u_{C^{\prime}}+\nabla_{C^{\prime}} u_{C}}{2}\right)-P_{F}^{E}\left(K_{E C}-\nabla_{E} u_{C}\right)\left(K_{C^{\prime}}^{F}-\nabla^{F} u_{C^{\prime}}\right)\right] \\
& +\psi^{-D} P_{A}^{C} P_{B}^{C^{\prime}} \bar{R}_{F C C^{\prime} D} O^{D} O^{F} \\
= & \psi^{-D} \mathfrak{t}_{A B}
\end{aligned}
$$

Where,

$$
\begin{aligned}
\mathfrak{t}_{A B}=P_{A}^{C} P_{B}^{D}[ & +\bar{R}_{F C D E} O^{E} O^{F}+\frac{K}{D}\left(K_{C D}-\frac{\nabla_{C} u_{D}+\nabla_{D} u_{C}}{2}\right) \\
& \left.-P^{E F}\left(K_{E C}-\nabla_{E} u_{C}\right)\left(K_{F D}-\nabla_{F} u_{D}\right)\right]
\end{aligned}
$$

In deriving (A.42) we have used the following identity

$$
P_{A}^{C}\left(\nabla_{D} O_{C}\right)=P_{D}^{E} P_{A}^{C}\left(\nabla_{E} O_{C}\right)-O_{D}\left[P_{A}^{C}(u \cdot \nabla) O_{C}\right]
$$

Which follows from the subsidiary condition.

\section{B Some identities}

In this appendix we shall prove some of the identities that we have used to compute the metric correction.

\section{B.1 The derivation of the identity (4.3)}

$$
\begin{aligned}
& {\left[\mathfrak{t}_{1}\right]_{C C^{\prime}}=P_{C}^{A} P_{C^{\prime}}^{B}\left[\frac{K}{D}\left(K_{A B}-\frac{\nabla_{A} u_{B}+\nabla_{B} u_{A}}{2}\right)-P_{E}^{D}\left(K_{D A}-\nabla_{D} u_{A}\right)\left(K_{B}^{E}-\nabla^{E} u_{B}\right)\right]} \\
& \nabla^{C}\left[\mathfrak{t}_{1}\right]_{C C^{\prime}} \\
& =\underbrace{\frac{K}{D} \nabla^{C}\left(P_{C}^{A} P_{C^{\prime}}^{B} K_{A B}\right)}_{\text {Term-1 }}-\underbrace{\left[\nabla^{C}\left\{P_{C}^{A} P_{F}^{D}\left(K_{D A}-\nabla_{D} u_{A}\right)\right\}\right]\left[P_{C^{\prime}}^{B} P^{E F}\left(K_{E B}-\nabla_{E} u_{B}\right)\right]}_{\text {Term- } 2} \\
& -\underbrace{\frac{K}{D} \nabla^{C}\left(P_{C}^{A} P_{C^{\prime}}^{B} \frac{\nabla_{A} u_{B}+\nabla_{B} u_{A}}{2}\right)}_{\text {Term-3 }} \\
& -\underbrace{\left[P_{C}^{A} P_{F}^{D}\left(K_{D A}-\nabla_{D} u_{A}\right)\right]\left[\nabla^{C}\left\{P_{C^{\prime}}^{B} P^{E F}\left(K_{E B}-\nabla_{E} u_{B}\right)\right\}\right]}_{\text {Term-4 }}
\end{aligned}
$$


After a bit of straight forward calculation the each of the above terms become

$$
\begin{aligned}
\text { Term- } 1 & \equiv \frac{K}{D} P_{C^{\prime}}^{E} \nabla_{E} K \\
\text { Term- } 2 & \equiv P^{E A} P_{C^{\prime}}^{B}\left[\nabla_{E} K-K\left(u^{D} K_{D E}\right)\right]\left(K_{A B}-\nabla_{A} u_{B}\right) \\
\text { Term- } 3 & \equiv \frac{K}{2 D} P_{C^{\prime}}^{E}\left[\nabla_{E} K+K(u \cdot \nabla) u_{E}\right] \\
\text { Term- } 4 & \equiv \frac{K}{D} P_{C^{\prime}}^{F}\left[K u^{D} K_{D F}-K(u \cdot \nabla) u_{F}\right]
\end{aligned}
$$

Adding (B.3), (B.4), (B.5) and (B.6) we get

$$
\begin{aligned}
\nabla^{C}\left[\mathfrak{t}_{1}\right]_{C C^{\prime}}= & \frac{K}{2 D} P_{C}^{B}\left[\nabla_{B} K+K(u \cdot \nabla) u_{B}-2 K\left(u^{A} K_{A B}\right)\right] \\
& -P^{B D} P_{C}^{A}\left(\nabla_{B} K-K\left(u^{E} K_{E B}\right)\right)\left[K_{D A}-\nabla_{D} u_{A}\right]
\end{aligned}
$$

\section{B.2 The derivation of scalar structure $\mathfrak{s}_{2}(3.16)$}

The scalar structure $\mathfrak{s}_{2}$ is defined as

$$
\begin{aligned}
& \mathfrak{s}_{2}=\frac{\nabla \cdot \mathfrak{v}}{D} \\
& \mathfrak{v}_{A}=P_{A}^{B}\left[\frac{K}{D}\left(n^{D} u^{E} O^{F} \bar{R}_{F B D E}\right)+\frac{K^{2}}{2 D^{2}}\left(\frac{\nabla_{B} K}{K}+(u \cdot \nabla) u_{B}-2 u^{D} K_{D B}\right)\right. \\
&\left.\quad-P^{F D}\left(\frac{\nabla_{F} K}{D}-\frac{K}{D}\left(u^{E} K_{E F}\right)\right)\left(K_{D B}-\nabla_{D} u_{B}\right)\right]
\end{aligned}
$$

Now,

$$
\begin{aligned}
\nabla^{A} \mathfrak{v}_{A}= & -K\left[\frac{K^{2}}{2 D^{2}}\left(\frac{(n \cdot \nabla) K}{K}+n^{B}(u \cdot \nabla) u_{B}\right)-P^{F D}\left(\frac{\nabla_{F} K}{D}-\frac{K}{D} u^{E} K_{E F}\right)\left(-n^{B} \nabla_{D} u_{B}\right)\right. \\
& \left.+\frac{K}{D} n^{D} u^{E} O^{F} n^{B} \bar{R}_{F B D E}\right]+P_{A}^{B}\left[\frac{K^{2}}{2 D^{2}}\left(\frac{\nabla^{A} \nabla_{B} K}{K}+\nabla^{A}\left[(u \cdot \nabla) u_{B}\right]-2 u^{D} \nabla^{A} K_{D B}\right)\right. \\
& -\left(\nabla^{A} P^{F D}\right)\left(\frac{\nabla_{F} K}{D}\right) K_{D B}-P^{F D}\left(\frac{\nabla^{A} \nabla_{F} K}{D}-\frac{K}{D} u^{E} \nabla^{A}\left(K_{E F}\right)\right) K_{D B} \\
& \left.-P^{F D}\left(\frac{\nabla_{F} K}{D}-\frac{K}{D}\left(u^{E} K_{E F}\right)\right)\left(\nabla^{A} K_{D B}-\nabla^{A} \nabla_{D} u_{B}\right)+\frac{K}{D}\left(K^{A D}\right) u^{E} O^{F} \bar{R}_{F B D E}\right] \\
= & \frac{K^{2}}{D}\left[-\frac{K}{2 D}\left(\frac{(n \cdot \nabla) K}{K}-u \cdot K \cdot u\right)+P^{F D}\left(\frac{\nabla_{F} K}{K}-u^{E} K_{E F}\right)\left(u^{B} \nabla_{D} n_{B}\right)\right. \\
& +n^{D} u^{E} u^{F} n^{B} \bar{R}_{F B D E}+\frac{1}{2 D}\left(\frac{\nabla^{2} K}{K}\right)-\frac{\lambda}{2}-\frac{1}{D}\left(u^{D} \nabla^{A} K_{D A}\right)+\frac{K}{D}\left(\frac{(n \cdot \nabla) K}{K}\right) \\
& \left.-P_{A}^{F} \frac{1}{D}\left(\frac{\nabla^{A} \nabla_{F} K}{K}-u^{E} \nabla^{A} K_{E F}\right)-P^{F D}\left(\frac{\nabla_{F} K}{K}-u^{E} K_{E F}\right)\left(\frac{\nabla^{A} K_{D A}}{K}\right)-\lambda\right]
\end{aligned}
$$


Now using

$$
\begin{aligned}
\frac{\nabla^{2} K}{K^{2}} & =\frac{\tilde{\nabla}^{2} K}{K^{2}}+\frac{(n \cdot \nabla) K}{K}+\mathcal{O}\left(\frac{1}{D}\right) \\
\text { and, } \quad \frac{\tilde{\nabla}^{2} K}{K^{2}} & =2\left(\frac{u \cdot \nabla K}{K}\right)-u \cdot K \cdot u+\frac{\lambda(D-1)}{K}
\end{aligned}
$$

We get the final expression

$$
\begin{aligned}
\nabla^{A} \mathfrak{v}_{A}=\frac{K^{2}}{D} & {\left[n^{B} n^{D} u^{E} u^{F} \bar{R}_{F B D E}-\frac{K}{D}\left(\frac{u \cdot \nabla K}{K}-u \cdot K \cdot u\right)-2 \lambda\right.} \\
& -(u \cdot K \cdot K \cdot u)+2\left(\frac{\nabla_{A} K}{K}\right) u^{B} K_{B}^{A}-\left(\frac{u \cdot \nabla K}{K}\right)^{2} \\
& \left.+2\left(\frac{u \cdot \nabla K}{K}\right)(u \cdot K \cdot u)-\left(\frac{\tilde{\nabla}^{D} K}{K}\right)\left(\frac{\tilde{\nabla}_{D} K}{K}\right)-(u \cdot K \cdot u)^{2}\right] \\
= & D \mathfrak{s}_{2}
\end{aligned}
$$

\section{B.3 The derivation of the identity (A.9)}

$$
\begin{aligned}
\nabla \cdot u & =\tilde{\nabla} \cdot u-\frac{(u \cdot \nabla) K}{K}-\frac{1}{K}(u \cdot \nabla)\left(\frac{(n \cdot \nabla) K}{K}\right)+\frac{1}{K} \frac{(n \cdot \nabla) K}{K} \frac{(u \cdot \nabla) K}{K} \\
\nabla \cdot u & =\tilde{\nabla} \cdot u+n_{B}(n \cdot \nabla) u^{B} \\
& =\tilde{\nabla} \cdot u-u^{B}\left[\psi K+\psi \frac{(n \cdot \nabla) N}{N}-N\right]^{-1} \tilde{\nabla}_{B}\left[\psi K+\psi \frac{(n \cdot \nabla) N}{N}-N\right]
\end{aligned}
$$

In the last line we have used the following relation

$$
\begin{aligned}
N D & =\psi K+\psi \frac{(n \cdot \nabla) N}{N}-N \\
\nabla \cdot u & =\tilde{\nabla} \cdot u-u^{B}\left[\psi K+\psi \frac{(n \cdot \nabla) N}{N}-N\right]^{-1} \tilde{\nabla}_{B}\left[\psi K+\psi \frac{(n \cdot \nabla) N}{N}-N\right] \\
& =\tilde{\nabla} \cdot u-\left[1-\frac{(n \cdot \nabla) N}{N K}+\frac{N}{\psi K}\right]\left[\frac{(u \cdot \nabla) K}{K}+\frac{1}{K}(u \cdot \nabla)\left\{\frac{(n \cdot \nabla) N}{N}-\frac{N}{\psi}\right\}\right](\mathrm{B} .16) \\
& =\tilde{\nabla} \cdot u-\frac{(u \cdot \nabla) K}{K}-\frac{1}{K}(u \cdot \nabla)\left\{\frac{(n \cdot \nabla) N}{N}-\frac{N}{\psi}\right\}+\left[\frac{(n \cdot \nabla) N}{N K}-\frac{N}{\psi K}\right] \frac{(u \cdot \nabla) K}{K} \\
& =\tilde{\nabla} \cdot u-\frac{(u \cdot \nabla) K}{K}-\frac{1}{K}(u \cdot \nabla)\left[\frac{(n \cdot \nabla) K}{K}\right]+\frac{1}{K}\left(\frac{(n \cdot \nabla) K}{K}\right)\left(\frac{(u \cdot \nabla) K}{K}\right)
\end{aligned}
$$

In the last line we have used

$$
\frac{(n \cdot \nabla) N}{N}=\frac{(n \cdot \nabla) K}{K}+\frac{K}{D}
$$




\section{B.4 The derivation of the identity (A.38)}

$$
\begin{aligned}
&(n \cdot \nabla) {\left[P_{D}^{C}\left\{2 K(u \cdot \nabla) O_{C}-\nabla^{2} O_{C}\right\}\right] } \\
&=(n \cdot \nabla)\left[P_{D}^{C}\left\{-2 K(n \cdot \nabla) u_{C}+\nabla^{2} u_{C}\right\}\right] \\
&= \underbrace{\left[(n \cdot \nabla) P_{D}^{C}\right]\left[-2 K(n \cdot \nabla) u_{C}+\nabla^{2} u_{C}\right]}_{1 \text { st Term }}+\underbrace{P_{D}^{C}(n \cdot \nabla)\left[-2 K(n \cdot \nabla) u_{C}+\nabla^{2} u_{C}\right]}_{2 \text { nd Term }} \\
& \mathbf{1} \text { st Term } \equiv\left[(n \cdot \nabla) P_{D}^{C}\right]\left[-2 K(n \cdot \nabla) u_{C}+\nabla^{2} u_{C}\right] \\
&=-n_{D}\left[(n \cdot \nabla) n^{C}\right]\left[-2 K(n \cdot \nabla) u_{C}+\nabla^{2} u_{C}\right]+u_{D}\left[(n \cdot \nabla) u^{C}\right]\left[-2 K(n \cdot \nabla) u_{C}+\nabla^{2} u_{C}\right] \\
&-\left[(n \cdot \nabla) n_{D}\right]\left[-2 K n^{C}(n \cdot \nabla) u_{C}+n^{C} \nabla^{2} u_{C}\right] \\
&= 0
\end{aligned}
$$

Where, we have used

$$
\begin{aligned}
(n \cdot \nabla) n_{D} & =-u_{D}\left[u^{B}(n \cdot \nabla) n_{B}\right]+P_{D}^{B}(n \cdot \nabla) n_{B} \\
(n \cdot \nabla) u_{D} & =n_{D}\left[n^{B}(n \cdot \nabla) u_{B}\right]+P_{D}^{B}(n \cdot \nabla) u_{B} \\
\text { And, }-2 K(n \cdot \nabla) u_{C}+\nabla^{2} u_{C} & =n_{C}\left[2 K u_{D}(n \cdot \nabla) n^{D}-u_{D} \nabla^{2} n^{D}\right]
\end{aligned}
$$

The third one follows from the fact that,

$$
\begin{aligned}
P_{B}^{C}[ & \left.-2 K(n \cdot \nabla) u_{C}+\nabla^{2} u_{C}\right] \\
& =P_{B}^{C}\left[\tilde{\nabla}^{2} u_{C}-K(n \cdot \nabla) u_{C}\right] \\
& =P_{B}^{C}\left[\tilde{\nabla}^{2} u_{C}-\tilde{\nabla}_{C} K-K(u \cdot \nabla) u_{C}+K u^{D} K_{D C}\right] \\
& =0
\end{aligned}
$$

Where, $\left[E_{1}\right]_{B}^{\text {vector }}$ is the leading order membrane equation.

$$
\begin{aligned}
2 \text { nd Term } & \equiv P_{D}^{C}(n \cdot \nabla)\left[-2 K(n \cdot \nabla) u_{C}+\nabla^{2} u_{C}\right] \\
& =P_{D}^{C}\left\{-2[(n \cdot \nabla) K]\left[(n \cdot \nabla) u_{C}\right]-2 K(n \cdot \nabla)\left[(n \cdot \nabla) u_{C}\right]+(n \cdot \nabla)\left(\nabla^{2} u_{C}\right)\right\}
\end{aligned}
$$

Now,

$$
\begin{aligned}
& P_{D}^{C}(n \cdot \nabla)\left(\nabla^{2} u_{C}\right) \\
& =P_{D}^{C} n^{E} \nabla_{E} \nabla_{F} \nabla^{F} u_{C} \\
& =P_{D}^{C} n^{E}\left[\nabla_{E}, \nabla_{F}\right] \nabla^{F} u_{C}+P_{D}^{C} n^{E} \nabla_{F} \nabla_{E} \nabla^{F} u_{C} \\
& =P_{D}^{C}\left[-\lambda(D-1)(n \cdot \nabla) u_{C}+n^{E} \bar{R}_{E F C B}\left(\nabla^{F} u^{B}\right)+n^{E} \nabla^{F}\left[\nabla_{E}, \nabla_{F}\right] u_{C}+n^{E} \nabla^{F} \nabla_{F} \nabla_{E} u_{C}\right] \\
& =P_{D}^{C}\left[-\lambda(D-1)(n \cdot \nabla) u_{C}+n^{E} \bar{R}_{E F C B}\left(\nabla^{F} u^{B}\right)+n^{E} u^{B}\left(\nabla^{F} \bar{R}_{E F C B}\right)+n^{E} \bar{R}_{E F C B}\left(\nabla^{F} u^{B}\right)\right. \\
& \left.\quad+\tilde{\nabla}^{2}\left[(n \cdot \nabla) u_{C}\right]-\left(\nabla^{2} n^{E}\right)\left(\nabla_{E} u_{C}\right)-2\left(\nabla_{F} n^{E}\right)\left(\nabla^{F} \nabla_{E} u_{C}\right)+K(n \cdot \nabla)\left[(n \cdot \nabla) u_{C}\right]\right] \\
& =P_{D}^{C}\left[\tilde{\nabla}^{2}\left[(n \cdot \nabla) u_{C}\right]-\left(\nabla^{2} n^{E}\right)\left(\nabla_{E} u_{C}\right)-2\left(\nabla_{F} n^{E}\right)\left(\nabla^{F} \nabla_{E} u_{C}\right)+K(n \cdot \nabla)\left[(n \cdot \nabla) u_{C}\right]\right.
\end{aligned}
$$




$$
\begin{aligned}
&\left.-\lambda(D-1)(n \cdot \nabla) u_{C}\right] \\
&=P_{D}^{C}\left[\frac{\tilde{\nabla}^{2} \tilde{\nabla}^{2} u_{C}}{K}-\frac{1}{K^{2}}\left(\tilde{\nabla}^{2} K\right) \tilde{\nabla}^{2} u_{C}-\left(\tilde{\nabla}^{2} n_{C}\right) \frac{u \cdot \nabla K}{K}-\left(\nabla^{2} n^{E}\right)\left(\nabla_{E} u_{C}\right)\right. \\
&\left.-2\left(\nabla_{F} n^{E}\right)\left(\nabla^{F} \nabla_{E} u_{C}\right)+K(n \cdot \nabla)\left[(n \cdot \nabla) u_{C}\right]-\lambda(D-1)(n \cdot \nabla) u_{C}\right]
\end{aligned}
$$

In the last line we have used,

$$
\begin{aligned}
P_{D}^{C} \tilde{\nabla}^{2}\left[(n \cdot \nabla) u_{C}\right] & =P_{D}^{C} \tilde{\nabla}^{2}\left[P_{C}^{E} \frac{\tilde{\nabla}^{2} u_{E}}{K}-n_{C} \frac{u \cdot \nabla K}{K}\right] \\
& =P_{D}^{C}\left[\frac{\tilde{\nabla}^{2} \tilde{\nabla}^{2} u_{C}}{K}-\frac{1}{K^{2}}\left(\tilde{\nabla}^{2} K\right) \tilde{\nabla}^{2} u_{C}-\left(\tilde{\nabla}^{2} n_{C}\right) \frac{u \cdot \nabla K}{K}\right]
\end{aligned}
$$

Using (B.23) in (B.22) we get,

\section{2-nd Term}

$$
\begin{aligned}
= & -P_{D}^{C} \lambda(D-1)(n \cdot \nabla) u_{C}+P_{D}^{C}\left[-2[(n \cdot \nabla) K]\left[(n \cdot \nabla) u_{C}\right]-K(n \cdot \nabla)\left[(n \cdot \nabla) u_{C}\right]+\frac{\tilde{\nabla}^{2} \tilde{\nabla}^{2} u_{C}}{K}\right. \\
& \left.-\frac{1}{K^{2}}\left(\tilde{\nabla}^{2} K\right) \tilde{\nabla}^{2} u_{C}-\left(\tilde{\nabla}^{2} n_{C}\right) \frac{u \cdot \nabla K}{K}-\left(\nabla^{2} n^{E}\right)\left(\nabla_{E} u_{C}\right)-2\left(\nabla_{D} n^{E}\right)\left(\nabla^{D} \nabla_{E} u_{C}\right)\right]
\end{aligned}
$$

Using the following identity whose derivation is a bit lengthy, and we are skipping the derivation

$$
\begin{aligned}
& P_{B}^{C}(n \cdot \nabla)\left[(n \cdot \nabla) u_{C}\right] \\
& =P_{B}^{C}\left[-4 \frac{u \cdot \nabla K}{K}\left[(u \cdot \nabla) u_{C}\right]+\left[(u \cdot \nabla) u_{C}\right](u \cdot K \cdot u)-7 \frac{u \cdot \nabla K}{K} \frac{\nabla_{C} K}{K}+\frac{\tilde{\nabla}^{2} \tilde{\nabla}^{2} u_{C}}{K^{2}}\right. \\
& +3(u \cdot K \cdot u) \frac{\nabla_{C} K}{K}-\frac{K}{D} u^{D} K_{D C}+4\left(u^{D} K_{D C}\right) \frac{u \cdot \nabla K}{K}-u^{D} K_{D C}(u \cdot K \cdot u)-2 K_{C}^{D} \frac{\nabla_{D} K}{K} \\
& \left.\quad-2\left(u_{E} K^{E D}\right)\left(\nabla_{D} u_{C}\right)+2 K^{A F} K_{A C} u_{F}-2 \frac{\lambda(D-1)}{K} \frac{\tilde{\nabla}^{2} u_{C}}{K}-2 u^{F} n^{E} O^{A} \bar{R}_{E F C A}\right]
\end{aligned}
$$

Now

\section{2-nd Term}

$$
\begin{aligned}
= & P_{B}^{C}\left[-\frac{K^{2}}{D}\left((u \cdot \nabla) u_{C}-u^{D} K_{D C}+\frac{\nabla_{C} K}{K}\right)\right]+P_{B}^{C} K\left[2 u^{F} n^{E} O^{A} \bar{R}_{E F C A}\right. \\
& +2 K_{C}^{D} \frac{\nabla_{D} K}{K}+2\left(u_{E} K^{E D}\right)\left(\nabla_{D} u_{C}\right)-2 K^{A F} K_{A C} u_{F}-2 \frac{\tilde{\nabla}^{E} K}{K}\left(\nabla_{E} u_{C}\right) \\
& \left.-2 \frac{u \cdot \nabla K}{K}(u \cdot \nabla) u_{C}+2 \frac{u \cdot \nabla K}{K} u^{D} K_{D C}+2(u \cdot K \cdot u)\left[(u \cdot \nabla) u_{C}\right]-2(u \cdot K \cdot u)\left(u^{D} K_{D C}\right)\right] \\
= & -2 D \mathfrak{v}_{B}
\end{aligned}
$$

Finally, we get

$$
(n \cdot \nabla)\left[P_{D}^{C}\left\{2 K(u \cdot \nabla) O_{C}-\nabla^{2} O_{C}\right\}\right]=-2 D \mathfrak{v}_{D}
$$




\section{B.5 The derivation of the identity (A.39)}

We can divide the L.H.S. of (A.39) as follows

$$
\begin{aligned}
P_{B}^{C} & {\left[2 K(u \cdot \nabla) O_{C}-\nabla^{2} O_{C}+2 n^{D} O^{E} u^{F} \bar{R}_{E C F D}+2 \frac{(n \cdot \nabla) K}{K}\left[(u \cdot \nabla) O_{C}\right]+\frac{\nabla_{C} K}{D}\right.} \\
& \left.-2\left(\nabla_{F} O_{C}\right)\left(u^{D} \nabla^{F} n_{D}\right)+\frac{K}{D}(u \cdot \nabla) O_{C}+\frac{K}{D} u^{D} K_{C D}\right] \equiv P_{A}^{C} \nabla^{2} u_{C}-P_{A}^{C} \nabla^{2} n_{C}+W
\end{aligned}
$$

where $W$ is what we get by subtracting off $P_{A}^{C} \nabla^{2} u_{C}-P_{A}^{C} \nabla^{2} n_{C}$ from the 1.h.s. of equation (B.29).

First we shall simpify $W$

$$
\begin{aligned}
W=P_{B}^{C} & {\left[2 K(u \cdot \nabla) O_{C}+2 n^{D} O^{E} u^{F} \bar{R}_{E C F D}+2 \frac{(n \cdot \nabla) K}{K}\left[(u \cdot \nabla) O_{C}\right]+\frac{\nabla_{C} K}{D}\right.} \\
& \left.-2\left(\nabla_{F} O_{C}\right)\left(u^{D} \nabla^{F} n_{D}\right)+\frac{K}{D}(u \cdot \nabla) O_{C}+\frac{K}{D} u^{D} K_{C D}\right] \\
=P_{B}^{C} & {\left[2 K\left(u^{D} K_{D C}\right)-2 K(u \cdot \nabla) u_{C}+2 u^{D} K_{D C}\left(\frac{(u \cdot \nabla) K}{K}-u \cdot K \cdot u\right)\right.} \\
& -2\left[(u \cdot \nabla) u_{C}\right]\left(\frac{(u \cdot \nabla) K}{K}-u \cdot K \cdot u\right)+\frac{\nabla_{C} K}{D}-2 u_{D} K_{F C} K^{F D} \\
+ & \left.2\left(\nabla_{F} u_{C}\right)\left(u_{D} K^{F D}\right)+\frac{K}{D}\left[(u \cdot \nabla) u_{C}\right]+2 n^{D} O^{E} u^{F} \bar{R}_{E C F D}\right]
\end{aligned}
$$

Now, we shall simplify $P_{A}^{C} \nabla^{2} n_{C}$

$$
\begin{aligned}
P_{B}^{C} \nabla^{2} n_{C} & =P_{B}^{C} \nabla^{D}\left(\nabla_{D} n_{C}\right) \\
& =P_{B}^{C} \nabla^{D}\left[K_{D C}+n_{D}(n \cdot \nabla) n_{C}\right] \\
& =\underbrace{P_{B}^{C} \nabla^{D} K_{D C}}_{T_{1}}+\underbrace{P_{B}^{C} K(n \cdot \nabla) n_{C}}_{T_{2}}+\underbrace{P_{B}^{C}(n \cdot \nabla)\left[(n \cdot \nabla) n_{C}\right]}_{T_{3}} \\
T_{1} & \equiv P_{B}^{C} \nabla^{D} K_{D C} \\
& =P_{B}^{C} \nabla^{D} K_{C D} \\
& =P_{B}^{C} \nabla^{D}\left(\Pi_{C}^{E} \nabla_{E} n_{D}\right) \\
& =P_{B}^{C}\left[\left(\nabla^{D} \Pi_{C}^{E}\right)\left(\nabla_{E} n_{D}\right)+\Pi_{C}^{E}\left(\nabla^{D} \nabla_{E} n_{D}\right)\right] \\
& =P_{B}^{C}\left\{-\left(\nabla^{D} n_{C}\right)\left[(n \cdot \nabla) n_{D}\right]+\Pi_{C}^{E} \nabla_{E} \nabla^{D} n_{D}\right\}+P_{B}^{E}\left[\nabla_{D}, \nabla_{E}\right] n^{D} \\
& =-P_{B}^{C} K_{C}^{D}\left(\frac{\nabla_{D} K}{K}\right)+P_{B}^{C} \nabla_{C} K-P_{B}^{E} \bar{R}_{D E C}{ }_{n}^{C} \\
& =-P_{B}^{C} K_{C}^{D}\left(\frac{\nabla_{D} K}{K}\right)+P_{B}^{C} \nabla_{C} K \\
T_{2} & \equiv P_{B}^{C} K\left[(n \cdot \nabla) n_{C}\right] \\
& =P_{B}^{C} K \frac{\nabla_{C}(N D)}{N D} \\
& =P_{B}^{C} K \frac{1}{\psi K+\psi \frac{(n \cdot \nabla) N}{N}-N} \nabla_{C}\left(\psi K+\psi \frac{(n \cdot \nabla) N}{N}-N\right)
\end{aligned}
$$




$$
\begin{aligned}
& =P_{B}^{C}\left(1-\frac{(n \cdot \nabla) N}{N K}+\frac{N}{\psi K}\right) \nabla_{C}\left(K+\frac{(n \cdot \nabla) N}{N}-\frac{N}{\psi}\right) \\
& =P_{B}^{C} \nabla_{C}\left(K+\frac{(n \cdot \nabla) N}{N}-\frac{N}{\psi}\right)+P_{B}^{C}\left(-\frac{(n \cdot \nabla) N}{N K}+\frac{N}{\psi K}\right) \nabla_{C} K \\
& =P_{B}^{C} \nabla_{C} K+P_{B}^{C} \nabla_{C}\left(\frac{(n \cdot \nabla) K}{K}\right)-P_{B}^{C}\left(\frac{(n \cdot \nabla) K}{K}\right)\left(\frac{\nabla_{C} K}{K}\right)
\end{aligned}
$$

In the first line we have used

$$
N D=\psi K+\psi \frac{(n \cdot \nabla) N}{N}-N
$$

And, in the last line we have used

$$
\begin{aligned}
\frac{(n \cdot \nabla) N=}{N}= & \frac{(n \cdot \nabla) K}{K}+\frac{K}{D} \\
T_{3} \equiv & P_{B}^{C}(n \cdot \nabla)\left[(n \cdot \nabla) n_{C}\right] \\
= & P_{B}^{C}\left[(n \cdot \nabla) \Pi_{C}^{D}\right]\left(\frac{\nabla_{D} N}{N}\right)+P_{B}^{C}(n \cdot \nabla)\left(\frac{\nabla_{C} N}{N}\right) \\
= & -P_{B}^{C}\left[(n \cdot \nabla) n_{C}\right]\left(\frac{(n \cdot \nabla) N}{N}\right)-P_{B}^{C} \frac{1}{N^{2}}[(n \cdot \nabla) N]\left(\nabla_{C} N\right)+P_{B}^{C} \frac{1}{N}\left[(n \cdot \nabla)\left(\nabla_{C} N\right)\right] \\
= & -P_{B}^{C}\left(\frac{\nabla_{C} K}{K}\right)\left(\frac{(n \cdot \nabla) N}{N}\right)-P_{B}^{C}\left(\frac{(n \cdot \nabla) N}{N}\right)\left(\frac{\nabla_{C} K}{K}\right)+P_{B}^{C} \frac{1}{N} n^{D} \nabla_{C} \nabla_{D} N \\
= & -2 P_{B}^{C}\left(\frac{\nabla_{C} K}{K}\right)\left(\frac{(n \cdot \nabla) N}{N}\right)+P_{B}^{C} \frac{1}{N} \nabla_{C}[(n \cdot \nabla) N]-P_{B}^{C} \frac{1}{N}\left(\nabla_{C} n^{D}\right)\left(\nabla_{D} N\right) \\
= & -2 P_{B}^{C}\left(\frac{\nabla_{C} K}{K}\right)\left(\frac{(n \cdot \nabla) N}{N}\right)+P_{B}^{C} \nabla_{C}\left(\frac{(n \cdot \nabla) N}{N}\right)+\frac{1}{N^{2}} P_{B}^{C}\left(\nabla_{C} N\right)[(n \cdot \nabla) N] \\
& -P_{B}^{C} \frac{1}{N}\left(\nabla_{C} n^{D}\right)\left(\nabla_{D} N\right) \\
= & -2 P_{B}^{C}\left(\frac{\nabla_{C} K}{K}\right)\left(\frac{(n \cdot \nabla) K}{K}+\frac{K}{D}\right)+P_{B}^{C} \nabla_{C}\left(\frac{(n \cdot \nabla) K}{K}+\frac{K}{D}\right) \\
& +P_{B}^{C}\left(\frac{\nabla_{C} K}{K}\right)\left(\frac{(n \cdot \nabla) K}{K}+\frac{K}{D}\right)-P_{B}^{C} K_{C}^{D}\left(\frac{\nabla_{D} K}{K}\right) \\
= & -2 P_{B}^{C}\left(\frac{\nabla_{C} K}{K}\right)\left(2 \frac{(u \cdot \nabla) K}{K}-u \cdot K \cdot u\right)+P_{B}^{C} \nabla_{C}\left(\frac{\tilde{\nabla}^{2} K}{K^{2}}\right)+P_{B}^{C} \frac{\nabla_{C} K}{K} \frac{\lambda(D-1)}{K} \\
& +P_{B}^{C}\left(\frac{\nabla_{C} K}{K}\right)\left(2 \frac{(u \cdot \nabla) K}{K}-u \cdot K \cdot u\right)-P_{B}^{C} K_{C}^{D} \frac{\nabla_{D} K}{K}
\end{aligned}
$$

In the last line we have used

$$
\frac{(n \cdot \nabla) K}{K}=\frac{\tilde{\nabla}^{2} K}{K^{2}}-\frac{(D-1) \lambda}{K}-\frac{K}{D}
$$

And, divergence of leading order vector membrane equation

$$
\frac{\tilde{\nabla}^{2} K}{K^{2}}=2 \frac{u \cdot \nabla K}{K}-u \cdot K \cdot u+\frac{\lambda(D-1)}{K}
$$


Adding (B.32) (B.33) and (B.36) we get

$$
\begin{aligned}
P_{B}^{C} \nabla^{2} n_{C}=P_{B}^{C} & {\left[2 \nabla_{C} K-2 K_{C}^{D}\left(\frac{\nabla_{D} K}{K}\right)+\frac{2}{K^{2}} \nabla_{C}\left(\tilde{\nabla}^{2} K\right)-2 \frac{\nabla_{C} K}{K} \frac{\lambda(D-1)}{K}\right.} \\
& \left.-6\left(\frac{\nabla_{C} K}{K}\right)\left(2 \frac{(u \cdot \nabla) K}{K}-u \cdot K \cdot u\right)\right]
\end{aligned}
$$

Now, we shall simplify $P_{B}^{C} \nabla^{2} u_{C}$

$$
\begin{aligned}
& P_{B}^{C} \tilde{\nabla}^{2} u_{C} \\
& =P_{B}^{C} \tilde{\nabla}^{E}\left(\Pi_{E}^{F} \Pi_{C}^{D} \nabla_{F} u_{D}\right) \\
& =P_{B}^{C} \Pi_{M}^{E} \nabla^{M}\left(\Pi_{E}^{F} \Pi_{C}^{D} \nabla_{F} u_{D}\right) \\
& =P_{B}^{C} \Pi_{M}^{N}\left(\nabla^{M} \Pi_{N}^{F}\right)\left(\nabla_{F} u_{C}\right)+P_{B}^{C} \Pi_{M}^{F}\left(\nabla^{M} \Pi_{C}^{D}\right)\left(\nabla_{F} u_{D}\right)+P_{B}^{C} \Pi_{M}^{F} \nabla^{M} \nabla_{F} u_{C} \\
& =P_{B}^{C}\left[-\Pi_{M}^{N} n^{F}\left(\nabla^{M} n_{N}\right)\left(\nabla_{F} u_{C}\right)-\Pi_{M}^{F} n^{D}\left(\nabla^{M} n_{C}\right)\left(\nabla_{F} u_{D}\right)+\nabla^{2} u_{C}-n^{F} n_{M} \nabla^{M} \nabla_{F} u_{C}\right] \\
& =P_{B}^{C}\left[-n^{F} K\left(\nabla_{F} u_{C}\right)-n^{D}\left(\nabla^{M} n_{C}\right)\left(\nabla_{M} u_{D}\right)+n^{D}\left[(n \cdot \nabla) n_{C}\right]\left[(n \cdot \nabla) u_{D}\right]\right. \\
& \left.\quad+\nabla^{2} u_{C}-n_{M} \nabla^{M}\left(n^{F} \nabla_{F} u_{C}\right)+n_{M}\left(\nabla^{M} n^{F}\right)\left(\nabla_{F} u_{C}\right)\right] \\
& =P_{B}^{C}\left[-K\left[(n \cdot \nabla) u_{C}\right]-\left(\nabla^{M} n_{C}\right)\left(n^{D} \nabla_{M} u_{D}\right)+\left[(n \cdot \nabla) n_{C}\right]\left[n^{D}(n \cdot \nabla) u_{D}\right]\right. \\
& \left.\quad+\nabla^{2} u_{C}-(n \cdot \nabla)\left[(n \cdot \nabla) u_{C}\right]+\left[(n \cdot \nabla) n^{F}\right]\left(\nabla_{F} u_{C}\right)\right] \\
& \Rightarrow P_{B}^{C} \nabla^{2} u_{C}=P_{B}^{C}\left[\tilde{\nabla}^{2} u_{C}+K\left[(n \cdot \nabla) u_{C}\right]+\left(\nabla^{M} n_{C}\right)\left(n^{D} \nabla_{M} u_{D}\right)\right. \\
& \left.\quad-\left[(n \cdot \nabla) n_{C}\right]\left[n^{D}(n \cdot \nabla) u_{D}\right]+(n \cdot \nabla)\left[(n \cdot \nabla) u_{C}\right]-\left[(n \cdot \nabla) n^{F}\right]\left(\nabla_{F} u_{C}\right)\right] \quad(\mathrm{B} .40)
\end{aligned}
$$

Now, $\quad P_{B}^{C}\left(\nabla^{M} n_{C}\right)\left(n^{D} \nabla_{M} u_{D}\right)$

$$
\begin{aligned}
& =-P_{B}^{C}\left[K_{C}^{M}+n^{M}(n \cdot \nabla) n_{C}\right]\left[u_{D} K_{M}^{D}+u_{D} n_{M}(n \cdot \nabla) n^{D}\right] \\
& =-P_{B}^{C} K_{C}^{M} K_{M}^{D} u_{D}-P_{B}^{C}\left[(n \cdot \nabla) n_{C}\right]\left[u^{D}(n \cdot \nabla) n_{D}\right] \\
& =-P_{B}^{C} K_{C}^{M} K_{M}^{D} u_{D}-P_{B}^{C} \frac{\nabla_{C} K}{K}\left(\frac{u \cdot \hat{\nabla} K}{K}\right)
\end{aligned}
$$

Putting (B.41) in (B.40) we get

$$
\begin{aligned}
P_{B}^{C} \nabla^{2} u_{C}= & P_{B}^{C} \tilde{\nabla}^{2} u_{C}+P_{B}^{C} K\left[(n \cdot \nabla) u_{C}\right]-P_{B}^{C} K_{C}^{M} K_{M}^{D} u_{D} \frac{P_{B}^{C} \frac{\nabla_{C} K}{K}\left(\frac{u \cdot \nabla K}{K}\right)}{} \\
& +P_{B}^{C} \frac{\nabla_{C} K}{K}\left(\frac{u \cdot \nabla K}{K}\right)+P_{B}^{C}(n \cdot \nabla)\left[(n \cdot \nabla) u_{C}\right]-P_{B}^{C} \frac{\tilde{\nabla}^{F} K}{K}\left(\nabla_{F} u_{C}\right) \\
\Rightarrow P_{B}^{C} \nabla^{2} u_{C}= & P_{B}^{C} \tilde{\nabla}^{2} u_{C}+P_{B}^{C} K\left[(n \cdot \nabla) u_{C}\right]-P_{B}^{C} K_{C}^{M} K_{M}^{D} u_{D}+P_{B}^{C}(n \cdot \nabla)\left[(n \cdot \nabla) u_{C}\right] \\
& -P_{B}^{C} \frac{\tilde{\nabla}^{F} K}{K}\left(\nabla_{F} u_{C}\right)
\end{aligned}
$$


As we have mentioned before derivation of $P_{B}^{C}(n \cdot \nabla)\left[(n \cdot \nabla) u_{C}\right]$ is lengthy, we shall use the result mentioned in eq (B.26).

Using (B.26) for $P_{B}^{C}(n \cdot \nabla)\left[(n \cdot \nabla) u_{C}\right]$ we get the final expression for $P_{B}^{C} \nabla^{2} u_{C}$

$$
\begin{aligned}
& P_{B}^{C} \nabla^{2} u_{C}=P_{B}^{C}\left[\tilde{\nabla}^{2} u_{C}+K\left[(n \cdot \nabla) u_{C}\right]-4 \frac{(u \cdot \nabla) K}{K}\left[(u \cdot \nabla) u_{C}\right]+\left[(u \cdot \nabla) u_{C}\right](u \cdot K \cdot u)\right. \\
& -7\left(\frac{u \cdot \nabla K}{K}\right) \frac{\nabla_{C} K}{K}-\frac{\tilde{\nabla}_{D} K}{K}\left(\nabla^{D} u_{C}\right)+3(u \cdot K \cdot u) \frac{\nabla_{C} K}{K}+\frac{\tilde{\nabla}^{2} \tilde{\nabla}^{2} u_{C}}{K^{2}}-\frac{K}{D} u^{D} K_{D C} \\
& \quad+4\left(u^{D} K_{D C}\right) \frac{u \cdot \nabla K}{K}-u^{D} K_{D C}(u \cdot K \cdot u)-2 K_{C}^{D} \frac{\nabla_{D} K}{K}-2\left(u_{E} K^{E D}\right)\left(\nabla_{D} u_{C}\right) \\
& \left.\quad+K^{A F} K_{A C} u_{F}-2 \frac{(D-1) \lambda}{K}\left(\frac{\nabla_{C} K}{K}-u^{E} K_{E C}+(u \cdot \nabla) u_{C}\right)-2 n^{E} u^{F} O^{A} \bar{R}_{E F C A}\right]
\end{aligned}
$$

Adding (B.30) (B.39) and (B.43) we get the final expression

$$
\frac{1}{K}\left(P_{B}^{C} \nabla^{2} u_{C}-P_{B}^{C} \nabla^{2} n_{C}+W\right)
$$

$=\left[\frac{\tilde{\nabla}^{2} u_{C}}{K}-\frac{\tilde{\nabla}_{C} K}{K}+u^{E} K_{E C}-u \cdot \tilde{\nabla} u_{C}\right] P_{B}^{C}+\left[\frac{\tilde{\nabla}^{2} \tilde{\nabla}^{2} u_{C}}{K^{3}}-\frac{u^{E} K_{E D} K_{C}^{D}}{K}-\frac{\left(\tilde{\nabla}_{C} K\right)(u \cdot \tilde{\nabla} K)}{K^{3}}\right.$

$-\frac{\left(\tilde{\nabla}_{E} K\right)\left(\tilde{\nabla}^{E} u_{C}\right)}{K^{2}}-\frac{2 K^{D E} \tilde{\nabla}_{D} \tilde{\nabla}_{E} u_{C}}{K^{2}}-\frac{\tilde{\nabla}_{C} \tilde{\nabla}^{2} K}{K^{3}}+\frac{\tilde{\nabla}_{C}\left(K_{E D} K^{E D} K\right)}{K^{3}}+3 \frac{(u \cdot K \cdot u)\left(u \cdot \tilde{\nabla} u_{C}\right)}{K}$

$-3 \frac{(u \cdot K \cdot u)\left(u^{E} K_{E C}\right)}{K}-6 \frac{(u \cdot \tilde{\nabla} K)\left(u \cdot \tilde{\nabla} u_{C}\right)}{K^{2}}+6 \frac{(u \cdot \tilde{\nabla} K)\left(u^{E} K_{E C}\right)}{K^{2}}+3 \frac{u \cdot \tilde{\nabla} u_{C}}{D-3}$

$\left.-3 \frac{u^{E} K_{E C}}{D-3}-\frac{(D-1) \lambda}{K^{2}}\left(\frac{\tilde{\nabla}_{C} K}{K}-2 u^{D} K_{D C}+2(u \cdot \tilde{\nabla}) u_{C}\right)\right] P_{B}^{C}$

$\equiv E_{B}^{\text {vector }}$

Where, in the last step we have used the following identity

$$
\begin{aligned}
P_{B}^{C}(n \cdot \nabla) u_{C}=P_{B}^{C}[ & \frac{\nabla_{C} K}{K}+\frac{1}{K} \nabla_{C}\left(\frac{\tilde{\nabla}^{2} K}{K^{2}}-\frac{(D-1) \lambda}{K}-\frac{K}{D}\right)-u^{D} K_{D C}+(u \cdot \nabla) u_{C} \\
& \left.-\frac{1}{K}\left(\frac{\nabla_{C} K}{K}\right)\left(2 \frac{(u \cdot \nabla) K}{K}-u \cdot K \cdot u-\frac{K}{D}\right)\right]
\end{aligned}
$$

\section{QNM for AdS/dS Schwarzschild Black hole: details of the calculation}

In this subsection we shall present several computational details. We shall follow [3] and [1]. Steps are tedious but a straightforward extension of what has been done in [1].

The answers for non-zero components of Christoffel symbols for metric (4.4) are (denoting the metric on unit sphere by $\bar{g}_{a b}$, its Christoffel symbols by $\bar{\Gamma}_{b c}^{a}$ and the covariant derivatives with respect to $\bar{g}_{a b}$ by $\bar{\nabla}_{a}$ )

$$
\begin{array}{lll}
\Gamma_{a b}^{r}=-r\left(1-\frac{\sigma r^{2}}{L^{2}}\right) \bar{g}_{a b}, & \Gamma_{r b}^{a}=\frac{1}{r} \delta_{b}^{a}, & \Gamma_{t t}^{r}=-r\left(1-\frac{\sigma r^{2}}{L^{2}}\right) \frac{\sigma}{L^{2}} \\
\Gamma_{r t}^{t}=-r\left(1-\frac{\sigma r^{2}}{L^{2}}\right)^{-1} \frac{\sigma}{L^{2}}, & \Gamma_{r r}^{r}=r\left(1-\frac{\sigma r^{2}}{L^{2}}\right)^{-1} \frac{\sigma}{L^{2}}, & \Gamma_{b c}^{a}=\bar{\Gamma}_{b c}^{a}
\end{array}
$$


The normal to membrane evaluates to

$$
n_{r}=\left(1-\frac{\sigma r^{2}}{L^{2}}\right)^{-\frac{1}{2}}, n_{t}=\left(1-\frac{\sigma r^{2}}{L^{2}}\right)^{-\frac{1}{2}}\left(-\epsilon \partial_{t} \delta r\right), n_{a}=\left(1-\frac{\sigma r^{2}}{L^{2}}\right)^{-\frac{1}{2}}\left(-\epsilon \bar{\nabla}_{a} \delta r\right)
$$

$\nabla_{A} n_{B}$ evaluates to

$$
\begin{aligned}
& \nabla_{r} n_{r}=0, \quad \nabla_{r} n_{t}=\left(1-\frac{\sigma r^{2}}{L^{2}}\right)^{-\frac{3}{2}} \frac{2 \sigma r}{L^{2}}\left(-\epsilon \partial_{t} \delta r\right) \\
& \nabla_{t} n_{r}=\left(1-\frac{\sigma r^{2}}{L^{2}}\right)^{-\frac{3}{2}} \frac{\sigma r}{L^{2}}\left(-\epsilon \partial_{t} \delta r\right), \quad \nabla_{a} n_{t}=\left(-\epsilon \partial_{t} \bar{\nabla}_{a} \delta r\right)\left(1-\frac{\sigma r^{2}}{L^{2}}\right)^{-\frac{1}{2}} \\
& \nabla_{t} n_{t}=\left(1-\frac{\sigma r^{2}}{L^{2}}\right)^{-\frac{1}{2}}\left(-\epsilon \partial_{t}^{2} \delta r\right)+\left(1-\frac{\sigma r^{2}}{L^{2}}\right)^{\frac{1}{2}} \frac{\sigma r}{L^{2}} \\
& \nabla_{r} n_{a}=\left(-\epsilon \bar{\nabla}_{a} \delta r\right)\left[\frac{\sigma r}{L^{2}}\left(1-\frac{\sigma r^{2}}{L^{2}}\right)^{-\frac{3}{2}}-\frac{1}{r}\left(1-\frac{\sigma r^{2}}{L^{2}}\right)^{-\frac{1}{2}}\right] \\
& \nabla_{a} n_{r}=\left(\epsilon \bar{\nabla}_{a} \delta r\right) \frac{1}{r}\left(1-\frac{\sigma r^{2}}{L^{2}}\right)^{-\frac{1}{2}}, \quad \nabla_{t} n_{a}=\left(-\epsilon \partial_{t} \bar{\nabla}_{a} \delta r\right)\left(1-\frac{\sigma r^{2}}{L^{2}}\right)^{-\frac{1}{2}} \\
& \nabla_{a} n_{b}=\left(1-\frac{\sigma r^{2}}{L^{2}}\right)^{-\frac{1}{2}}\left(-\epsilon \bar{\nabla}_{a} \bar{\nabla}_{b} \delta r\right)+r\left(1-\frac{\sigma r^{2}}{L^{2}}\right)^{\frac{1}{2}} \bar{g}_{a b}
\end{aligned}
$$

The projector $P_{A}^{B}=\delta_{B}^{A}-n^{A} n_{B}$ evaluates to

$$
\begin{array}{ll}
P_{r}^{r}=0, & P_{t}^{t}=1, \\
P_{t}^{r}=\epsilon \partial_{t} \delta r, & P_{r}^{t}=\left(1-\frac{\sigma r^{2}}{L^{2}}\right)^{-2}\left(-\epsilon \partial_{t} \delta r\right), \\
P_{a}^{r}=\epsilon \bar{\nabla}_{a} \delta r, & P_{r}^{a}=\frac{1}{r^{2}}\left(1-\frac{\sigma r^{2}}{L^{2}}\right)^{-1}\left(\epsilon \bar{\nabla}^{a} \delta r\right)
\end{array}
$$

The spacetime form of Extrinsic curvature $K_{A B}=\Pi_{A}^{C} \nabla_{C} n_{B}$ evaluates to

$$
\begin{aligned}
& K_{r r}=0, \quad K_{r t}=\left(1-\frac{\sigma r^{2}}{L^{2}}\right)^{-\frac{3}{2}} \frac{\sigma r}{L^{2}}\left(-\epsilon \partial_{t} \delta r\right), \quad K_{r a}=\frac{1}{r}\left(1-\frac{\sigma r^{2}}{L^{2}}\right)^{-\frac{1}{2}}\left(\epsilon \bar{\nabla}_{a} \delta r\right) \\
& K_{t a}=\left(1-\frac{\sigma r^{2}}{L^{2}}\right)^{-\frac{1}{2}}\left(-\epsilon \partial_{t} \bar{\nabla}_{a} \delta r\right), \quad K_{t t}=\left(1-\frac{\sigma r^{2}}{L^{2}}\right)^{-\frac{1}{2}}\left(-\epsilon \partial_{t}^{2} \delta r\right)+\left(1-\frac{\sigma r^{2}}{L^{2}}\right)^{\frac{1}{2}} \frac{\sigma r}{L^{2}} \\
& K_{a b}=\left(1-\frac{\sigma r^{2}}{L^{2}}\right)^{-\frac{1}{2}}\left(-\epsilon \bar{\nabla}_{a} \bar{\nabla}_{b} \delta r\right)+r\left(1-\frac{\sigma r^{2}}{L^{2}}\right)^{\frac{1}{2}} \bar{g}_{a b}
\end{aligned}
$$

Answers for the nonzero components of Christoffel symbols for metric (4.9) are

$$
\begin{aligned}
\Gamma_{t t}^{t} & =-\left(1-\frac{\sigma}{L^{2}}\right)^{-1} \frac{\sigma}{L^{2}}\left(\epsilon \partial_{t} \delta r\right), & \Gamma_{t t}^{a} & =-\frac{\sigma}{L^{2}}\left(\epsilon \bar{\nabla}^{a} \delta r\right) \\
\Gamma_{a t}^{t} & =-\left(1-\frac{\sigma}{L^{2}}\right)^{-1} \frac{\sigma}{L^{2}}\left(\epsilon \bar{\nabla}_{a} \delta r\right), & \Gamma_{a b}^{t} & =\left(1-\frac{\sigma}{L^{2}}\right)^{-1}\left(\epsilon \partial_{t} \delta r\right) \bar{g}_{a b} \\
\Gamma_{t b}^{a} & =\left(\epsilon \partial_{t} \delta r\right) \delta_{b}^{a}, & \Gamma_{b c}^{a} & =\bar{\Gamma}_{b c}^{a}+\epsilon\left(\bar{\nabla}_{b} \delta r \delta_{c}^{a}+\bar{\nabla}_{c} \delta r \delta_{b}^{a}-\bar{\nabla}^{a} \delta r \bar{g}_{b c}\right)
\end{aligned}
$$


$\hat{\nabla}_{\mu} u_{\nu}$ evaluates to

$$
\begin{aligned}
& \hat{\nabla}_{t} u_{t}=0, \quad \hat{\nabla}_{t} u_{a}=\epsilon \partial_{t} \delta u_{a}-\left(1-\frac{\sigma}{L^{2}}\right)^{-\frac{1}{2}}\left(\frac{\sigma}{L^{2}}\right)\left(\epsilon \bar{\nabla}_{a} \delta r\right) \\
& \hat{\nabla}_{a} u_{t}=0, \quad \hat{\nabla}_{a} u_{b}=\epsilon \bar{\nabla}_{a} \delta u_{b}+\left(1-\frac{\sigma}{L^{2}}\right)^{-\frac{1}{2}}\left(\epsilon \partial_{t} \delta r\right) \bar{g}_{a b}
\end{aligned}
$$

The projector $\mathcal{P}_{\nu}^{\mu} \equiv \delta_{\nu}^{\mu}+u^{\mu} u_{\nu}$ evaluates to

$$
\mathcal{P}_{t}^{t}=0, \quad \mathcal{P}_{t}^{a}=-\left(1-\frac{\sigma}{L^{2}}\right)^{\frac{1}{2}}\left(\epsilon \delta u_{a}\right), \quad \mathcal{P}_{a}^{t}=\left(1-\frac{\sigma}{L^{2}}\right)^{-\frac{1}{2}}\left(\epsilon \delta u_{a}\right), \quad \mathcal{P}_{b}^{a}=\delta_{b}^{a}
$$

\section{C.1 Computation of $\mathcal{K}_{\mu \nu}$}

We define $\mathcal{K}_{\mu \nu}$ as the pullback of Extrinsic curvature $K_{M N}$ (which is a spacetime tensor) on the membrane surface

$$
\mathcal{K}_{\mu \nu}=\left.\left(\frac{\partial X^{M}}{\partial y^{\mu}}\right)\left(\frac{\partial X^{N}}{\partial y^{\nu}}\right) K_{M N}\right|_{r=1+\epsilon \delta r}
$$

where we denote the coordinates in spacetime $\left(r, t, \theta^{a}\right)$ by $X^{M}$ and the coordinates on the membrane worldvolume $\left(t, \theta^{a}\right)$ by $y^{\mu}$. The extrinsic curvature $K_{A B}$ is defined as

$$
K_{A B}=\Pi_{A}^{C} \nabla_{C} n_{B}, \quad \text { where } \quad \Pi_{A C}=g_{A C}-n_{A} n_{C}
$$

Now equation (C.9) evaluated upto linear order for the QNM calculation implies that

$$
\mathcal{K}_{\mu \nu}=\epsilon\left(\partial_{\mu} \delta r\right) K_{r \nu}+\epsilon\left(\partial_{\nu} \delta r\right) K_{r \mu}+K_{\mu \nu}+\mathcal{O}\left(\epsilon^{2}\right)
$$

From (C.5) we see that $K_{r N}=\mathcal{O}(\epsilon)$. Using this fact along with (C.11) gives us

$$
\begin{aligned}
\mathcal{K}_{t t} & =\left(1-\frac{\sigma}{L^{2}}\right)^{-\frac{1}{2}}\left(-\epsilon \partial_{t}^{2} \delta r\right)+\left(1-\frac{\sigma}{L^{2}}\right)^{\frac{1}{2}}\left(\frac{\sigma}{L^{2}}\right)\left(1+\epsilon \delta r-\frac{\sigma \epsilon \delta r}{L^{2}-\sigma}\right) \\
\mathcal{K}_{t a} & =\left(1-\frac{\sigma}{L^{2}}\right)^{-\frac{1}{2}}\left(-\epsilon \partial_{t} \bar{\nabla}_{a} \delta r\right) \\
\mathcal{K}_{a b} & =\left(1-\frac{\sigma}{L^{2}}\right)^{-\frac{1}{2}}\left(-\epsilon \bar{\nabla}_{a} \bar{\nabla}_{b} \delta r\right)+\left(1-\frac{\sigma}{L^{2}}\right)^{\frac{1}{2}}\left(1+\epsilon \delta r-\frac{\sigma \epsilon \delta r}{L^{2}-\sigma}\right) \hat{g}_{a b}
\end{aligned}
$$

Trace of Extrinsic curvature (C.12) evaluates to

$$
\begin{aligned}
\mathcal{K}= & \left(1-\frac{\sigma}{L^{2}}\right)^{-\frac{3}{2}}\left(\epsilon \partial_{t}^{2} \delta r\right)-\left(1-\frac{\sigma}{L^{2}}\right)^{-\frac{1}{2}}\left(\frac{\sigma}{L^{2}}\right)\left(1+\frac{\epsilon L^{2} \delta r}{L^{2}-\sigma}\right) \\
& +\left(1-\frac{\sigma}{L^{2}}\right)^{-\frac{1}{2}}\left(-\epsilon \bar{\nabla}^{2} \delta r\right)+\left(1-\frac{\sigma}{L^{2}}\right)^{\frac{1}{2}}\left(1-\frac{\epsilon L^{2} \delta r}{L^{2}-\sigma}\right)(D-2)
\end{aligned}
$$




\section{C.2 Computation of the terms relevant for the membrane equation}

Here, we report the relevant terms needed to evaluate the membrane equation upto linear order. The relevant terms at leading order evaluate to

$$
\begin{aligned}
u^{\nu} \mathcal{K}_{\nu t}= & \frac{\sigma}{L^{2}}+\mathcal{O}(\epsilon) \\
u^{\nu} \mathcal{K}_{\nu a}= & \left(1-\frac{\sigma}{L^{2}}\right)^{-1}\left(-\epsilon \partial_{t} \bar{\nabla}_{a} \delta r\right)+\left(1-\frac{\sigma}{L^{2}}\right)^{\frac{1}{2}}\left(\epsilon \delta u_{a}\right) \\
u^{\nu} \hat{\nabla}_{\nu} u_{t}= & 0 \\
u^{\nu} \hat{\nabla}_{\nu} u_{a}= & \left(1-\frac{\sigma}{L^{2}}\right)^{-\frac{1}{2}}\left(\epsilon \partial_{t} \delta u_{a}\right)-\left(1-\frac{\sigma}{L^{2}}\right)^{-1} \frac{\sigma}{L^{2}}\left(\epsilon \bar{\nabla}_{a} \delta r\right) \\
\hat{\nabla}_{t} \mathcal{K}= & \mathcal{O}(\epsilon) \\
\hat{\nabla}_{a} \mathcal{K}= & \left(1-\frac{\sigma}{L^{2}}\right)^{-\frac{3}{2}}\left(\epsilon \partial_{t}^{2} \bar{\nabla}_{a} \delta r\right)-\left(1-\frac{\sigma}{L^{2}}\right)^{-\frac{3}{2}} \frac{\sigma}{L^{2}}\left(\epsilon \bar{\nabla}_{a} \delta r\right) \\
& +\left(1-\frac{\sigma}{L^{2}}\right)^{-\frac{1}{2}}\left(-\epsilon \bar{\nabla}_{a} \bar{\nabla}^{2} \delta r\right)-(D-2)\left(1-\frac{\sigma}{L^{2}}\right)^{-\frac{1}{2}}\left(\epsilon \bar{\nabla}_{a} \delta r\right) \\
\hat{\nabla}^{2} u_{t}= & \mathcal{O}(\epsilon) \\
\hat{\nabla}^{2} u_{a}= & -\left(1-\frac{\sigma}{L^{2}}\right)^{-1}\left(\epsilon \partial_{t}^{2} \delta u_{a}\right)+\left(1-\frac{\sigma}{L^{2}}\right)^{-\frac{3}{2}} \frac{\sigma}{L^{2}}\left(\epsilon \partial_{t} \bar{\nabla}_{a} \delta r\right) \\
& +\epsilon \bar{\nabla}^{2} \delta u_{a}+\left(1-\frac{\sigma}{L^{2}}\right)^{-\frac{1}{2}}\left(\epsilon \partial_{t} \bar{\nabla}_{a} \delta r\right)
\end{aligned}
$$

The relevant terms at subleading order evaluate to

$$
\begin{aligned}
u^{\nu} \mathcal{K}_{\nu \mu} \mathcal{K}_{t}^{\mu} & =-\left(\frac{\sigma}{L^{2}}\right)^{2}\left(1-\frac{\sigma}{L^{2}}\right)^{-\frac{1}{2}} \\
u^{\nu} \mathcal{K}_{\nu \mu} \mathcal{K}_{a}^{\mu} & =\left(1-\frac{\sigma}{L^{2}}\right)^{-\frac{3}{2}} \frac{\sigma}{L^{2}} \epsilon \partial_{t} \bar{\nabla}_{a} \delta r-\left(1-\frac{\sigma}{L^{2}}\right)^{-\frac{1}{2}} \epsilon \partial_{t} \bar{\nabla}_{a} \delta r+\left(1-\frac{\sigma}{L^{2}}\right) \epsilon \delta u_{a} \\
\hat{\nabla}^{2} \hat{\nabla}^{2} u_{t} & =\mathcal{O}(\epsilon) \\
\hat{\nabla}^{2} \hat{\nabla}^{2} u_{a} & =\bar{\nabla}^{2} \bar{\nabla}^{2} \delta u_{a} \\
u . \hat{\nabla} \mathcal{K} & =\mathcal{O}(\epsilon) \\
\hat{\nabla}^{\nu} \mathcal{K} \hat{\nabla}_{\nu} u_{t} & =\mathcal{O}(\epsilon) \\
\hat{\nabla}^{\nu} \mathcal{K} \hat{\nabla}_{\nu} u_{a} & =\mathcal{O}(\epsilon)^{2} \\
\mathcal{K}^{\mu \nu} \hat{\nabla}_{\mu} \hat{\nabla}_{\nu} u_{t} & =\mathcal{O}(\epsilon) \\
\mathcal{K}^{\mu \nu} \hat{\nabla}_{\mu} \hat{\nabla}_{\nu} u_{a} & =\left(1-\frac{\sigma}{L^{2}}\right)^{\frac{1}{2}} \epsilon \hat{\nabla}^{2} \delta u_{a} \\
\hat{\nabla}_{t} \hat{\nabla}^{2} \mathcal{K} & =\mathcal{O}(\epsilon) \\
\hat{\nabla}_{a} \hat{\nabla}^{2} \mathcal{K} & =-\left(1-\frac{\sigma}{L^{2}}\right)^{-\frac{1}{2}} \hat{\nabla}_{a} \hat{\nabla}^{2} \hat{\nabla}^{2} \delta r-(D-2)\left(1-\frac{\sigma}{L^{2}}\right)^{-\frac{1}{2}} \hat{\nabla}_{a} \hat{\nabla}^{2} \delta r \\
\hat{\nabla}_{t}\left(\mathcal{K}_{\mu \nu} \mathcal{K}^{\mu \nu} \mathcal{K}\right) & =\mathcal{O}(\epsilon) \\
\hat{\nabla}_{a}\left(\mathcal{K}_{\mu \nu} \mathcal{K}^{\mu \nu} \mathcal{K}\right) & =-3(D-2)\left(1-\frac{\sigma}{L^{2}}\right)^{\frac{1}{2}} \epsilon\left(\hat{\nabla}_{a} \hat{\nabla}^{2} \delta r+(D-2) \hat{\nabla}_{a} \delta r\right) \\
(u \cdot \mathcal{K} \cdot u) u^{\nu} \hat{\nabla}_{\nu} u_{t} & =\mathcal{O}(\epsilon)
\end{aligned}
$$




$$
\begin{aligned}
(u \cdot \mathcal{K} \cdot u) u^{\nu} \hat{\nabla}_{\nu} u_{a} & =\left(1-\frac{\sigma}{L^{2}}\right)^{-1} \frac{\sigma}{L^{2}} \epsilon \partial_{t} \delta u_{a}-\left(1-\frac{\sigma}{L^{2}}\right)^{-\frac{3}{2}}\left(\frac{\sigma}{L^{2}}\right)^{2} \epsilon \hat{\nabla}_{a} \delta r \\
(u \cdot \mathcal{K} \cdot u) u^{\mu} \mathcal{K}_{\mu t} & =\left(1-\frac{\sigma}{L^{2}}\right)^{-\frac{1}{2}}\left(\frac{\sigma}{L^{2}}\right)^{2} \\
(u \cdot \mathcal{K} \cdot u) u^{\mu} \mathcal{K}_{\mu a} & =-\left(1-\frac{\sigma}{L^{2}}\right)^{-\frac{3}{2}}\left(\frac{\sigma}{L^{2}}\right) \epsilon \partial_{t} \hat{\nabla}_{a} \delta r+\frac{\sigma}{L^{2}} \epsilon \delta u_{a} \\
(u \cdot \hat{\nabla} \mathcal{K}) u^{\mu} \mathcal{K}_{\mu t} & =\mathcal{O}(\epsilon) \\
(u \cdot \hat{\nabla} \mathcal{K}) u^{\mu} \mathcal{K}_{\mu a} & =\mathcal{O}(\epsilon)^{2} \\
(u \cdot \hat{\nabla} \mathcal{K}) u^{\nu} \hat{\nabla}_{\nu} u_{t} & =\mathcal{O}(\epsilon) \\
(u \cdot \hat{\nabla} \mathcal{K}) u^{\nu} \hat{\nabla}_{\nu} u_{a} & =\mathcal{O}(\epsilon)^{2}
\end{aligned}
$$

\section{C.3 Arguments leading to (4.13)}

Firstly, for convenience, rewrite the membrane equation (4.12) as

$$
E_{\mu}^{\text {tot }} \equiv \mathcal{P}_{\mu}^{\nu} E_{\nu}, \quad \text { where } \quad E_{\mu} \equiv \frac{\hat{\nabla}^{2} u_{\mu}}{\mathcal{K}}-\frac{\hat{\nabla}_{\mu} \mathcal{K}}{\mathcal{K}}+u^{\nu} \mathcal{K}_{\nu \mu}-u^{\nu} \hat{\nabla}_{\nu} u_{\mu}+\ldots
$$

So, we get

$$
\begin{aligned}
& E_{t}^{\mathrm{tot}}=E_{t} \mathcal{P}_{t}^{t}+E_{b} \mathcal{P}_{t}^{b} \\
& E_{a}^{\mathrm{tot}}=E_{t} \mathcal{P}_{a}^{t}+E_{b} \mathcal{P}_{a}^{b}
\end{aligned}
$$

We can see for a uniform membrane configuration with spherical symmetry that $E_{a}$ would be zero and hence we have $E_{a} \sim \mathcal{O}(\epsilon)$ in case of fluctuations. Also we see that $\mathcal{P}_{t}^{t}=0$ and $\mathcal{P}_{t}^{a} \sim \mathcal{O}(\epsilon)$. Hence we see from (C.16) that $E_{t}^{\text {tot }}$ is identically zero at the linear order. Similarly because $\mathcal{P}_{a}^{t}=\mathcal{O}(\epsilon)$, only $\mathcal{O}\left(\epsilon^{0}\right)$ pieces of $E_{t}$ are relevant for evaluating $E_{a}^{\text {tot }}$ at linear order. Hence in subsection C.2 we evaluated only those terms in $E_{\mu}$ that are relevant for the linearized analysis.

Substituting the expressions derived in subsection (C.2) in the linearized vector membrane equation in the angular directions we finally get (4.13).

\section{QNM for AdS Schwarzschild black brane: details of the calculation}

Just like previous section, here we shall provide the details of the computation required to determine the QNM frequencies for AdS Schwarzschild black brane.

The answers for nonzero components of Christoffel symbols for the background metric (4.28) are

$$
\Gamma_{r r}^{r}=\frac{-1}{r}, \quad \Gamma_{a b}^{r}=-r^{3} \delta_{a b}, \quad \Gamma_{r b}^{a}=\frac{1}{r} \delta_{b}^{a}, \quad \Gamma_{t t}^{r}=r^{3}, \quad \Gamma_{r t}^{t}=\frac{1}{r}
$$

Normal to the membrane evaluates to

$$
n_{r}=\frac{1}{r}, \quad n_{a}=\frac{-\epsilon \partial_{a} \delta r}{r}, \quad n_{t}=\frac{-\epsilon \partial_{t} \delta r}{r}
$$


Non zero components of $\nabla_{M} n_{N}$ evaluate to

$$
\begin{aligned}
& \nabla_{r} n_{r}=0, \quad \nabla_{r} n_{t}=\frac{2 \epsilon \partial_{t} \delta r}{r^{2}}, \quad \nabla_{t} n_{r}=\frac{\epsilon \partial_{t} \delta r}{r^{2}}, \quad \nabla_{t} n_{t}=-\frac{\epsilon \partial_{t}^{2} \delta r}{r}-r^{2}, \\
& \nabla_{r} n_{a}=\frac{2 \epsilon \partial_{a} \delta r}{r^{2}}, \quad \nabla_{a} n_{r}=\frac{\epsilon \partial_{a} \delta r}{r^{2}}, \quad \nabla_{t} n_{a}=\frac{-\epsilon \partial_{t} \partial_{a} \delta r}{r}, \\
& \nabla_{a} n_{t}=\frac{-\epsilon \partial_{t} \partial_{a} \delta r}{r}, \quad \nabla_{a} n_{b}=\frac{-\epsilon \partial_{a} \partial_{b} \delta r}{r}+r^{2} \delta_{a b}
\end{aligned}
$$

The projector $P_{A}^{B}=\delta_{A}^{B}-n_{A} n^{B}$ evaluates to

$$
\begin{array}{llll}
P_{r}^{r}=0, & P_{t}^{t}=1, & P_{b}^{a}=\delta_{b}^{a}, & P_{t}^{a}=0,
\end{array}
$$

Nonzero components of the spacetime form of Extrinsic curvature $K_{M N}$ evaluate to

$$
\begin{aligned}
& K_{r r}=0, \quad K_{r t}=\frac{\epsilon \partial_{t} \delta r}{r^{2}}, \quad K_{r a}=\frac{\epsilon \partial_{a} \delta r}{r^{2}} \\
& K_{t t}=\frac{-\epsilon \partial_{t}^{2} \delta r}{r}-r^{2}, \quad K_{t a}=\frac{-\epsilon \partial_{t} \partial_{a} \delta r}{r}, \quad K_{a b}=\frac{-\epsilon \partial_{a} \partial_{b} \delta r}{r}+r^{2} \delta_{a b}
\end{aligned}
$$

Nonzero components of Christoffel symbols for the induced metric (4.30) evaluate to

$$
\begin{array}{ll}
\Gamma_{t t}^{t}=\epsilon \partial_{t} \delta r, & \Gamma_{t t}^{a}=\epsilon \partial^{a} \delta r, \quad \Gamma_{a t}^{t}=\epsilon \partial_{a} \delta r, \quad \Gamma_{a b}^{t}=\epsilon \partial_{t} \delta r \delta_{a b} \\
\Gamma_{t b}^{a}=\epsilon \partial_{t} \delta r \delta_{b}^{a}, & \Gamma_{b c}^{a}=\epsilon\left(\partial_{b} \delta r \delta_{c}^{a}+\partial_{c} \delta r \delta_{b}^{a}-\partial^{a} \delta r \delta_{b c}\right)
\end{array}
$$

The projector $\mathcal{P}_{\nu}^{\mu}=\delta_{\nu}^{\mu}+u^{\mu} u_{\nu}$ evaluates to

$$
\mathcal{P}_{b}^{a}=\delta_{b}^{a}, \quad \mathcal{P}_{t}^{t}=0, \quad \mathcal{P}_{a}^{t}=\epsilon \delta u_{a}, \quad \mathcal{P}_{t}^{a}=-\epsilon \delta u_{a}
$$

Nonzero components of $\hat{\nabla}_{\mu} u_{\nu}$ evaluate to

$$
\begin{aligned}
\hat{\nabla}_{t} u_{t} & =0, & \hat{\nabla}_{t} u_{a}=\epsilon \partial_{t} \delta u_{a}+\epsilon \partial_{a} \delta r, & \hat{\nabla}_{a} u_{t}=0, \\
\hat{\nabla}_{a} u_{b} & =\epsilon \partial_{a} \delta u_{b}+\epsilon \partial_{t} \delta r \delta_{a b} & &
\end{aligned}
$$

\section{D.1 Computation of $\mathcal{K}_{\mu \nu}$}

As done previously, $\mathcal{K}_{\mu \nu}$ is defined as the pullback of spacetime form of extrinsic curvature $K_{M N}$ on the membrane worldvolume. Doing this procedure we find that the nonzero components of $\mathcal{K}_{\mu \nu}$ evaluate to

$$
\mathcal{K}_{t t}=-\epsilon \partial_{t}^{2} \delta r-(1+2 \epsilon \delta r), \quad \mathcal{K}_{t a}=-\epsilon \partial_{t} \partial_{a} \delta r, \quad \mathcal{K}_{a b}=-\epsilon \partial_{a} \partial_{b} \delta r+(1+2 \epsilon \delta r) \delta_{a b}
$$

Trace of Extrinsic curvature $\mathcal{K}_{\mu \nu}$ evaluates to

$$
\mathcal{K}=n+\epsilon \partial_{t}^{2} \delta r-\epsilon \partial_{a} \partial^{a} \delta r
$$

where we raised the index $a$ in (D.10) with $\delta^{a b}$. 


\section{D.2 Computation of the terms relevant for membrane equation}

At leading order the relevant terms evaluate to

$$
\begin{aligned}
u^{\nu} \mathcal{K}_{\nu t} & =-1+\mathcal{O}(\epsilon) \\
u^{\nu} \mathcal{K}_{\nu a} & =-\epsilon \partial_{t} \partial_{a} \delta r+\epsilon \delta u_{a} \\
u^{\nu} \hat{\nabla}_{\nu} u_{t} & =\mathcal{O}(\epsilon) \\
u^{\nu} \hat{\nabla}_{\nu} u_{a} & =\epsilon \partial_{t} \delta u_{a}+\epsilon \partial_{a} \delta r \\
\hat{\nabla}_{t} \mathcal{K} & =\mathcal{O}(\epsilon) \\
\hat{\nabla}_{a} \mathcal{K} & =\epsilon \partial_{a} \partial_{t}^{2} \delta r-\epsilon \partial_{a} \partial^{2} \delta r \\
\hat{\nabla}^{2} u_{t} & =\mathcal{O}(\epsilon) \\
\hat{\nabla}^{2} u_{a} & =-\epsilon \partial_{t}^{2} \delta u_{a}+\epsilon \partial^{2} \delta u_{a}
\end{aligned}
$$

While at subleading order the relevant terms evaluate to

$$
\begin{aligned}
u^{\nu} K_{\nu \mu} K_{t}^{\mu} & =-1+\mathcal{O}(\epsilon) \\
u^{\nu} K_{\nu \mu} K_{a}^{\mu} & =-2 \epsilon \partial_{t} \partial_{a} \delta r+\epsilon \delta u_{a} \\
\hat{\nabla}^{2} \hat{\nabla}^{2} u_{t} & =\mathcal{O}(\epsilon) \\
\hat{\nabla}^{2} \hat{\nabla}^{2} u_{a} & =\epsilon \partial_{t}^{4} \delta u_{a}-2 \epsilon \partial_{t}^{2} \partial^{2} \delta u_{a}+\epsilon \partial^{4} \delta u_{a} \\
u . \hat{\nabla} \mathcal{K} & =\mathcal{O}(\epsilon) \\
\hat{\nabla}^{\nu} \mathcal{K} \hat{\nabla}_{\nu} u_{t} & =\mathcal{O}(\epsilon) \\
\hat{\nabla}^{\nu} \mathcal{K} \hat{\nabla}_{\nu} u_{a} & =\mathcal{O}(\epsilon)^{2} \\
K^{\mu \nu} \hat{\nabla}_{\mu} \hat{\nabla}_{\nu} u_{t} & =\mathcal{O}(\epsilon) \\
K^{\mu \nu} \hat{\nabla}_{\mu} \hat{\nabla}_{\nu} u_{a} & =-\epsilon \partial_{t}^{2} \delta u_{a}+\epsilon \partial^{2} \delta u_{a} \\
\hat{\nabla}_{t} \hat{\nabla}^{2} \mathcal{K} & =\mathcal{O}(\epsilon) \\
\hat{\nabla}_{a} \hat{\nabla}^{2} \mathcal{K} & =-\epsilon \partial_{a} \partial_{t}^{4} \delta r+2 \epsilon \partial_{a} \partial_{t}^{2} \partial^{2} \delta r-\epsilon \partial_{a} \partial^{2} \partial^{2} \delta r \\
\hat{\nabla}_{t}\left(K_{\mu \nu} K^{\mu \nu} \mathcal{K}\right) & =\mathcal{O}(\epsilon) \\
\hat{\nabla}_{a}\left(K_{\mu \nu} K^{\mu \nu} \mathcal{K}\right) & =3 \epsilon\left(\partial_{a} \partial_{t}^{2} \delta r-\partial_{a} \partial^{2} \delta r\right) \\
(u . K . u) u^{\nu} \hat{\nabla}_{\nu} u_{t} & =\mathcal{O}(\epsilon) \\
(u . K . u) u^{\nu} \hat{\nabla}_{\nu} u_{a} & =-\left(\epsilon \partial_{t} \delta u_{a}+\epsilon \partial_{a} \delta r\right) \\
(u . K . u) u^{\mu} K_{\mu t} & =1+\mathcal{O}(\epsilon) \\
(u . K . u) u^{\mu} K_{\mu a} & =\epsilon \partial_{t} \partial_{a} \delta r-\epsilon \delta u_{a} \\
(u . \hat{\nabla} \mathcal{K}) u^{\mu} K_{\mu t} & =\mathcal{O}(\epsilon) \\
(u . \hat{\nabla} \mathcal{K}) u^{\mu} K_{\mu a} & =\mathcal{O}(\epsilon)^{2} \\
(u . \hat{\nabla} \mathcal{K}) u^{\nu} \hat{\nabla}_{\nu} u_{t} & =\mathcal{O}(\epsilon) \\
(u . \hat{\nabla} \mathcal{K}) u^{\nu} \hat{\nabla}_{\nu} u_{a} & =\mathcal{O}(\epsilon)^{2}
\end{aligned}
$$




\section{D.3 Arguments leading to (4.32)}

Following the same trick as previously done, we denote the vector membrane equation as

$$
E_{\mu}^{\text {tot }} \equiv \mathcal{P}_{\mu}^{\nu} E_{\nu}, \quad \text { where } \quad E_{\mu} \equiv\left[\frac{\hat{\nabla}^{2} u_{\alpha}}{\mathcal{K}}-\frac{\hat{\nabla}_{\alpha} \mathcal{K}}{\mathcal{K}}+u^{\beta} K_{\beta \alpha}-u \cdot \hat{\nabla} u_{\alpha}\right]+\ldots
$$

Hence we have

$$
\begin{aligned}
& E_{t}^{\mathrm{tot}}=E_{t} \mathcal{P}_{t}^{t}+E_{b} \mathcal{P}_{t}^{b} \\
& E_{a}^{\mathrm{tot}}=E_{t} \mathcal{P}_{a}^{t}+E_{b} \mathcal{P}_{a}^{b}
\end{aligned}
$$

For the uniform planar membrane we have translational symmetry along the $x_{a}$ directions, so we have $E_{b} \sim \mathcal{O}(\epsilon)$ in the case of fluctuations. Note that $\mathcal{P}_{t}^{t}=0, \mathcal{P}_{t}^{a} \sim \mathcal{O}(\epsilon)$ and also $E_{b} \sim \mathcal{O}(\epsilon)$, hence $E_{t}^{\text {tot }}$ vanishes upto linear order. Note that $\mathcal{P}_{a}^{t} \sim \mathcal{O}(\epsilon)$, hence only $\mathcal{O}\left(\epsilon^{0}\right)$ pieces of $E_{t}$ contribute when we evaluate $E_{a}^{\text {tot }}$ upto linear order. Keeping these facts in mind we calculated only those terms that are relevant in subsection D.2.

Substituting the expressions derived in subsection (D.2) in the linearized vector membrane equation in the angular directions we finally get (4.32).

Open Access. This article is distributed under the terms of the Creative Commons Attribution License (CC-BY 4.0), which permits any use, distribution and reproduction in any medium, provided the original author(s) and source are credited.

\section{References}

[1] S. Bhattacharyya, P. Biswas, B. Chakrabarty, Y. Dandekar and A. Dinda, The large D black hole dynamics in $A d S / d S$ backgrounds, JHEP 10 (2018) 033 [arXiv:1704.06076] [INSPIRE].

[2] S. Bhattacharyya, A. De, S. Minwalla, R. Mohan and A. Saha, A membrane paradigm at large D, JHEP 04 (2016) 076 [arXiv: 1504.06613] [INSPIRE].

[3] S. Bhattacharyya, M. Mandlik, S. Minwalla and S. Thakur, A charged membrane paradigm at large D, JHEP 04 (2016) 128 [arXiv:1511.03432] [INSPIRE].

[4] Y. Dandekar, A. De, S. Mazumdar, S. Minwalla and A. Saha, The large D black hole membrane paradigm at first subleading order, JHEP 12 (2016) 113 [arXiv:1607.06475] [INSPIRE].

[5] Y. Dandekar, S. Mazumdar, S. Minwalla and A. Saha, Unstable 'black branes' from scaled membranes at large D, JHEP 12 (2016) 140 [arXiv:1609.02912] [INSPIRE].

[6] S. Bhattacharyya et al., Currents and radiation from the large D black hole membrane, JHEP 05 (2017) 098 [arXiv: 1611.09310] [INSPIRE].

[7] Y. Dandekar, S. Kundu, S. Mazumdar, S. Minwalla, A. Mishra and A. Saha, An action for and hydrodynamics from the improved large D membrane, JHEP 09 (2018) 137 [arXiv: 1712.09400] [INSPIRE].

[8] R. Emparan, R. Suzuki and K. Tanabe, The large D limit of general relativity, JHEP 06 (2013) 009 [arXiv: 1302.6382] [InSPIRE].

[9] R. Emparan, D. Grumiller and K. Tanabe, Large-D gravity and low-D strings, Phys. Rev. Lett. 110 (2013) 251102 [arXiv:1303.1995] [INSPIRE]. 
[10] R. Emparan and K. Tanabe, Universal quasinormal modes of large D black holes, Phys. Rev. D 89 (2014) 064028 [arXiv:1401.1957] [InSPIRE].

[11] R. Emparan, R. Suzuki and K. Tanabe, Decoupling and non-decoupling dynamics of large D black holes, JHEP 07 (2014) 113 [arXiv:1406.1258] [InSPIRE].

[12] G. Giribet, Large D limit of dimensionally continued gravity, Phys. Rev. D 87 (2013) 107504 [arXiv: 1303.1982] [INSPIRE].

[13] P. Dominis Prester, Small black holes in the large D limit, JHEP 06 (2013) 070 [arXiv: 1304.7288] [INSPIRE].

[14] R. Emparan and K. Tanabe, Holographic superconductivity in the large $D$ expansion, JHEP 01 (2014) 145 [arXiv:1312.1108] [INSPIRE].

[15] R. Emparan, R. Suzuki and K. Tanabe, Instability of rotating black holes: large D analysis, JHEP 06 (2014) 106 [arXiv:1402.6215] [INSPIRE].

[16] R. Emparan, R. Suzuki and K. Tanabe, Quasinormal modes of (anti-)de Sitter black holes in the 1/D expansion, JHEP 04 (2015) 085 [arXiv: 1502.02820] [INSPIRE].

[17] A.M. García-García and A. Romero-Bermúdez, Conductivity and entanglement entropy of high dimensional holographic superconductors, JHEP 09 (2015) 033 [arXiv:1502.03616] [INSPIRE].

[18] R. Suzuki and K. Tanabe, Stationary black holes: large D analysis, JHEP 09 (2015) 193 [arXiv: 1505.01282] [INSPIRE].

[19] R. Suzuki and K. Tanabe, Non-uniform black strings and the critical dimension in the $1 / D$ expansion, JHEP 10 (2015) 107 [arXiv:1506.01890] [INSPIRE].

[20] R. Emparan, R. Suzuki and K. Tanabe, Evolution and end point of the black string instability: large D solution, Phys. Rev. Lett. 115 (2015) 091102 [arXiv:1506.06772] [INSPIRE].

[21] K. Tanabe, Black rings at large D, JHEP 02 (2016) 151 [arXiv: 1510.02200] [INSPIRE].

[22] K. Tanabe, Instability of the de Sitter Reissner-Nordstrom black hole in the 1/D expansion, Class. Quant. Grav. 33 (2016) 125016 [arXiv:1511.06059] [INSPIRE].

[23] B. Chen, Z.-Y. Fan, P. Li and W. Ye, Quasinormal modes of Gauss-Bonnet black holes at large D, JHEP 01 (2016) 085 [arXiv: 1511.08706] [INSPIRE].

[24] R. Emparan, K. Izumi, R. Luna, R. Suzuki and K. Tanabe, Hydro-elastic complementarity in black branes at large D, JHEP 06 (2016) 117 [arXiv:1602.05752] [INSPIRE].

[25] A. Sadhu and V. Suneeta, Nonspherically symmetric black string perturbations in the large dimension limit, Phys. Rev. D 93 (2016) 124002 [arXiv:1604.00595] [INSPIRE].

[26] C.P. Herzog, M. Spillane and A. Yarom, The holographic dual of a Riemann problem in a large number of dimensions, JHEP 08 (2016) 120 [arXiv:1605.01404] [INSPIRE].

[27] K. Tanabe, Elastic instability of black rings at large D, arXiv:1605.08116 [INSPIRE].

[28] K. Tanabe, Charged rotating black holes at large D, arXiv:1605.08854 [INSPIRE].

[29] M. Rozali and A. Vincart-Emard, On brane instabilities in the large D limit, JHEP 08 (2016) 166 [arXiv:1607.01747] [INSPIRE].

[30] B. Chen and P.-C. Li, Instability of charged Gauss-Bonnet black hole in de Sitter spacetime at large D, arXiv: 1607.04713 [INSPIRE]. 
[31] B. Chen, P.-C. Li and Z.-Z. Wang, Charged black rings at large D, JHEP 04 (2017) 167 [arXiv: 1702.00886] [INSPIRE].

[32] B. Chen and P.-C. Li, Static Gauss-Bonnet black holes at large D, JHEP 05 (2017) 025 [arXiv: 1703.06381] [INSPIRE].

[33] M. Rozali, E. Sabag and A. Yarom, Holographic turbulence in a large number of dimensions, JHEP 04 (2018) 065 [arXiv:1707.08973] [INSPIRE].

[34] B. Chen, P.-C. Li and C.-Y. Zhang, Einstein-Gauss-Bonnet black strings at large D, JHEP 10 (2017) 123 [arXiv:1707.09766] [INSPIRE].

[35] R. Emparan, R. Luna, M. Martínez, R. Suzuki and K. Tanabe, Phases and stability of non-uniform black strings, JHEP 05 (2018) 104 [arXiv: 1802.08191] [INSPIRE].

[36] C.P. Herzog and Y. Kim, The large dimension limit of a small black hole instability in anti-de Sitter space, JHEP 02 (2018) 167 [arXiv:1711.04865] [INSPIRE].

[37] B. Chen, P.-C. Li, Y. Tian and C.-Y. Zhang, Holographic turbulence in Einstein-Gauss-Bonnet gravity at large D, arXiv: 1804.05182 [INSPIRE].

[38] R.M. Wald, General relativity, University of Chicago Press, Chicago, IL, U.S.A., (1984). 